\title{
THE DYNAMICS OF ACUTE INFLAMMATION
}

by

\section{Rukmini Kumar}

M.Sc. Physics, Indian Institute of Technology, Madras, 1999

B.Sc. Physics, Stella Maris College, Madras, 1997

\author{
Submitted to the Graduate Faculty of \\ the Faculty of Arts and Sciences in partial fulfillment \\ of the requirements for the degree of \\ Doctor of Philosophy
}

University of Pittsburgh

2004 


\section{UNIVERSITY OF PITTSBURGH}

\section{FACULTY OF ARTS AND SCIENCES}

This dissertation was presented

by

Rukmini Kumar

It was defended on

September 2, 2004

and approved by

Prof. Carson Chow, Mathematics

Prof. David Jasnow, Physics

Prof. Donna Naples, Physics

Prof. Jonathan Rubin, Mathematics

Prof. Xiao-lun Wu, Physics

Dissertation Advisors: Prof. Carson Chow, Mathematics

Prof. David Jasnow, Physics 


\title{
THE DYNAMICS OF ACUTE INFLAMMATION
}

\author{
Rukmini Kumar, $\mathrm{PhD}$ \\ University of Pittsburgh, 2004
}

The acute inflammatory response is the non-specific and immediate reaction of the body to pathogenic organisms, tissue trauma and unregulated cell growth. An imbalance in this response could lead to a condition commonly known as "shock" or "sepsis". This thesis is an attempt to elucidate the dynamics of acute inflammatory response to infection and contribute to its systemic understanding through mathematical modeling and analysis.

The models of immunity discussed use Ordinary Differential Equations (ODEs) to model the variation of concentration in time of the various interacting species. Chapter 2 discusses three such models of increasing complexity. Sections 2.1 and 2.2 discuss smaller models that capture the core features of inflammation and offer general predictions concerning the design of the system. Phase-space and bifurcation analyses have been used to examine the behavior at various parameter regimes. Section 2.3 discusses a global physiological model that includes several equations modeling the concentration (or numbers) of cells, cytokines and other mediators. The conclusions drawn from the reduced and detailed models about the qualitative effects of the parameters are very similar and these similarities have also been discussed.

In Chapter 3, the specific applications of the biologically detailed model are discussed in greater detail. These include a simulation of anthrax infection and an in silico simulation of a clinical trial. Such simulations are very useful to biologists and could prove to be invaluable tools in drug design.

Finally, Chapter 4 discusses the general problem of extinction of populations modeled as continuous variables in ODEs is discussed. The average time to extinction and threshold 
are estimated based on analyzing the equivalent stochastic processes.

Keywords: Acute inflammation, ODE models, phase-space and bifurcation analyses, anthrax infection, in silico clinical trials, thresholds for extinction. 


\section{ACKNOWLEDGEMENTS}

My five years at graduate school have been an enjoyable time of learning and growth. I have been fortunate to know and work with some really special people to whom I owe a debt of gratitude.

Prof. Carson Chow has been my boss, advisor, teacher and friend. He has inspired me to push my limits and has always given me something to strive for. I am grateful to him for his generosity and guidance and hope to have more opportunities to learn from him in the future.

I have had a great experience working in a truly inter-disciplinary team. Dr. Gilles Clermont has shared his warmth and wisdom and Dr. Yoram Vodovotz, his energy and infectious optimism. I have had unfettered access to their time and knowledge and I sincerely appreciate that.

I thank my committee members - Prof. David Jasnow's valuable suggestions have definitely influenced the direction of my thesis. Prof. Jon Rubin's careful observations have motivated a more basic understanding of the limitations of the models. I thank Prof. Donna Naples for being a friend and mentor and Prof. Xiao-lun Wu for his time.

My time at Pitt began in the Physics Department. I thank the gang at $306 \mathrm{OEH}$ for some great and fun times, my girlfriends, Voica Radescu, Ummi Abbas, Michele Belfort and Yvette Blair for inviting me into their lives and for being an important part of mine, Pranay Goel for always asking me the tough questions on science and life and Sandeep Tyagi whom I could go to with my queries even when it was completely out of the blue.

Thackeray Hall has been my second home and I will miss all the friendly folk who dropped by to chat. Judy Day, my friend, colleague and co-traveler, I thank for her thoughtfulness and for all the candid discussions on work and life, my office-mate Yixin Guo for literally always being there, Samat Moldakarimov for his fascinating stories and Stephanie Hoogendorn for her friendship. I thank my cousin Dr.Chandran Kalyanam for his careful review of my manuscript.

My family has always been larger than my parents and sister and has included my aunts, grand-parents, cousins and friends. I thank them all for their often blind faith in my abilities 
and for their unalloyed affection. I also thank my aunt Shanthi for always asking the most of herself and for leading a life that is a constant source of inspiration. My husband Vikram is my rock and for 10 years now, has been the reason I wake up in the morning with a smile. 


\section{TABLE OF CONTENTS}

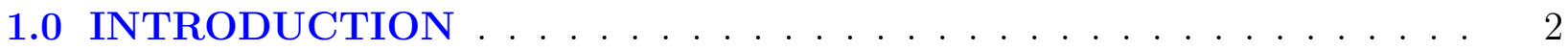

1.1 The problem of systemic inflammation . . . . . . . . . . . . . 2

1.1.1 Acute inflammation . . . . . . . . . . . . . . . . 2

1.1.2 Pathogenesis of systemic inflammation . . . . . . . . . . . . 4

1.1.3 Therapies for systemic inflammation . . . . . . . . . . . 5 5

1.2 Motivation and outline of the thesis . . . . . . . . . . . . . 6

2.0 DYNAMICS OF ACUTE INFLAMMATION - 3 MODELS OF INCREASING COMPLEXITY . . . . . . . . . . . . . . . . 9

2.1 Sepsis in two dimensions . . . . . . . . . . . . . . . . . . . . . . . . . . 9

2.2 A 3-d model of acute inflammation . . . . . . . . . . . . . . . . 12

2.2.1 Dynamics of the model - Numerical examples . . . . . . . . . . 15

2.2.2 Dynamics of the model - Fixed Points and Bifurcations . . . . . . . . 19

2.2 .32 parameter bifurcations . . . . . . . . . . . . 24

2.2.4 Discussion of the 3 -d model . . . . . . . . . . . . . . . . . . . . . 28

2.3 A global model of acute inflammation . . . . . . . . . . . . . 34

2.3.1 Features of the model . . . . . . . . . . . . . . . . 35

2.3 .2 Simulations . . . . . . . . . . . . . . . . . . . . 38

2.3.3 Connections . . . . . . . . . . . . . . . . . . . 39

2.4 Limitations of the models . . . . . . . . . . . . . . . . . . . 41

2.5 Appendix to the 2 -d model . . . . . . . . . . . . . . . . . . . . 49

2.6 Appendix to the $3-\mathrm{d}$ model $\ldots \ldots \ldots \ldots$. . . . . . . . . . . . . . . .

2.6.1 Eigenvalues of Fixed Points . . . . . . . . . . . . . . . . 50

vii 
$2.6 .1 .1 \mathrm{FP} 1 \ldots \ldots \ldots \ldots$

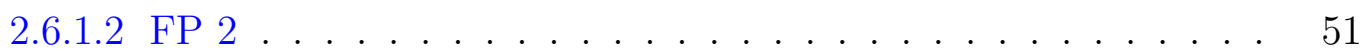

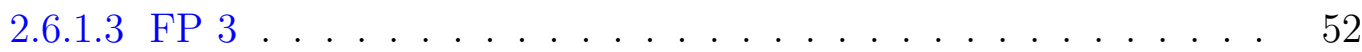

2.6.1.4 FP 4 and FP $5 \ldots \ldots \ldots \ldots$

2.6.2 Regimes for healthy response . . . . . . . . . . . . . . . . . . 54

2.6.2.1 Effect of varying the coupling curve . . . . . . . . . . . 55

2.7 Appendix to the Global Model . . . . . . . . . . . . . . . . 56

2.7.1 Table of mediators modeled . . . . . . . . . . . . . 56

2.7.2 Estimated values of parameters . . . . . . . . . . . . . 57

2.7 .3 The global model . . . . . . . . . . . . . . . . . . 58

3.0 APPLICATIONS OF A GLOBAL MODEL OF ACUTE INFLAMMATION - (I) ANTHRAX AND (II) CLINICAL TRIALS . . . . . . . . 60

3.1 Anthrax . . . . . . . . . . . . . . . . . . . . . 60 60

3.1.1 Pathogenesis of anthrax infection . . . . . . . . . . . . . . 61

3.1.2 Simulations from a model of anthrax infection . . . . . . . . . . 63

3.1.3 Treatment and prevention strategies: Current status . . . . . . . . . 65

3.1 .4 Predictions from the model . . . . . . . . . . . . . . 66

3.1.4.1 Vaccine against protective antigen alone may protect . . . . . 66

3.1.4.2 Combination therapy is the most ideal . . . . . . . . . 67

3.1.5 Discussion of the anthrax model . . . . . . . . . . . . . . . . . . . 69

3.2 Clinical Trials . . . . . . . . . . . . . . . . . . . . . . 72

3.2 .1 Design of the clinical trial . . . . . . . . . . . . . . . . 73

3.2.2 Results and Discussion . . . . . . . . . . . . . . . . 74

3.3 Appendix: Modification of the detailed model to simulate anthrax infection $\quad 77$

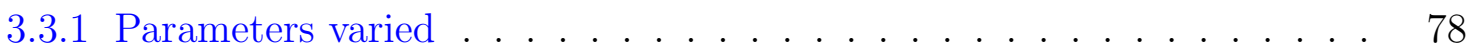

\subsection{THE THRESHOLD OF EXTINCTION OF DECAYING POPULA-} TIONS IN A DETERMINISTIC MODEL . . . . . . . . . . . . . 79

4.1 Threshold for the general decay process from the Master Equation and Gillespie simulation . . . . . . . . . . . . . . . . . . . . . 80 
4.2 Threshold for the simple decay process from the Fokker-Planck and Langevin

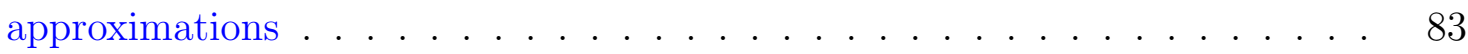

4.2.1 Fokker-Planck Equation . . . . . . . . . . . . . . . . . . 83

4.2 .2 Langevin Equation . . . . . . . . . . . . . . . . . 86

4.3 Threshold of extinction for larger models . . . . . . . . . . . . . . . . 88

4.4 Appendix . . . . . . . . . . . . . . . . . . . . . . . 93

4.4.1 $\langle T\rangle$ from the Master Equation . . . . . . . . . . . . . . . . 93

$4.4 .2\langle T\rangle$ from the Fokker-Planck Equation . . . . . . . . . . . . . . . 96

5.0 BIBLIOGRAPHY . . . . . . . . . . . . . . . . . . . . . . . . . . 99 


\section{LIST OF TABLES}

2.1 Mediators in the global model (Courtesy of Dr. G. Clermont) . . . . . . . . 56

2.2 Estimated values of parameters $\ldots \ldots \ldots \ldots \ldots \ldots \ldots \ldots \ldots \ldots \ldots \ldots \ldots \ldots \ldots \ldots \ldots \ldots$

2.3 Parameters used only in the case of specific interventions ( 0 in the base-line

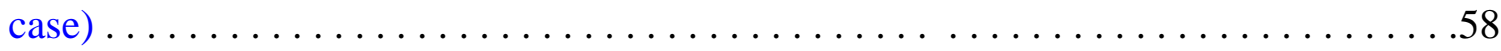

2.4 The various thresholds of activation $\ldots \ldots \ldots \ldots \ldots \ldots \ldots \ldots \ldots \ldots \ldots \ldots \ldots \ldots \ldots \ldots \ldots$

3.1 The rate constants are different in the anthrax model and the global model

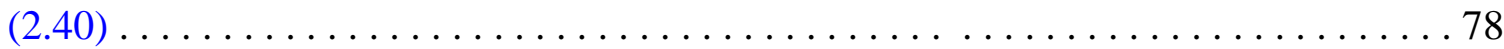




\section{LIST OF FIGURES}

2.1 The phase-plane for the 2-d model $(2.1,2.3)$ showing "healthy" and "shock" trajectories and the stable manifold that separates them ...............13

2.2 Time courses of $p, m$ and $l$ for the healthy response in the $3-d$ model ....................................... 17

2.3 Time course of $\mathrm{p}, \mathrm{m}$ and $\mathrm{l}$ for persistent non-infectious inflammation $\ldots \ldots \ldots 17$

2.4 Time course of $\mathrm{p}, \mathrm{m}$ and $\mathrm{l}$ for persistent infectious inflammation $\ldots \ldots \ldots \ldots$

2.5 Time course of $\mathrm{p}, \mathrm{m}$ and $\mathrm{l}$ for recurrent infection. ................. 18

2.6 Time course of $\mathrm{p}, \mathrm{m}$ and $\mathrm{l}$ for severe immuno-deficiency. . . . . . . . . . . . 19

2.7 Projection of fixed-point conditions onto the $p-m$ plane $\ldots \ldots \ldots \ldots \ldots \ldots 20$

2.8 Bifurcation plot showing outcome as $\mathrm{k}_{\mathrm{pm}}\left(\mathrm{a}=\mathrm{k}_{\mathrm{pm}} / \mathrm{k}_{\mathrm{p}}\right)$ is increased. ........ 25

2.9 Bifurcation plot showing outcome as $\mathrm{k}_{\mathrm{mp}}$ is increased. . . . . . . . . . . 26

2.10 Bifurcation plot showing outcome as $\mathrm{k}_{\operatorname{lm}}\left(\mathrm{b}=\mathrm{k}_{\mathrm{lm}} / \mathrm{k}_{\mathrm{l}}\right)$ is increased. . . . . . . 27

2.11 A 2 parameter bifurcation plot that varies $\mathrm{k}_{\mathrm{pm}}$ and $\mathrm{k}_{\mathrm{lm}} \ldots \ldots \ldots \ldots \ldots \ldots 29$ 
2.12 A 2 parameter bifurcation plot that varies $k_{m p}$ and $k_{l m} \ldots \ldots \ldots \ldots \ldots \ldots$

2.13 Bifurcation plots that vary coupling parameters $\theta$ and $w \ldots \ldots \ldots \ldots \ldots 31$

2.14 A 2 parameter bifurcation plot that varies $\theta$ and $w \ldots \ldots \ldots \ldots \ldots \ldots \ldots \ldots$

2.15 Simulation of a healthy inflammatory response in the global model . . . . . . . 42

2.16 Simulation of persistent non-infectious inflammation $\ldots \ldots \ldots \ldots \ldots \ldots .43$

2.17 Simulation of persistent infectious inflammation................. 44

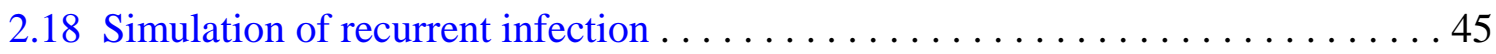

2.19 Simulation of severe immuno-deficiency $\ldots \ldots \ldots \ldots \ldots \ldots \ldots \ldots \ldots$

2.20 The bifurcation plot varying the strength of macrophage and neutrophil response to pathogen and tissue-factor response to endotoxin . . . . . . . . . . . . . . . 47

2.21 The bifurcation plot varying the strength of thrombin stimulation of damage.. . . 47

3.1 Modifications to account for anthrax-specific effects: ... . . . . . . . . . 68

3.2 Vaccination against protective antigen..$\ldots \ldots \ldots \ldots \ldots \ldots \ldots \ldots \ldots$ 
3.3 Simulation of the efficacy of antibiotics and vaccination. . . . . . . . . 70

3.4 Simulation of a set of parameters where vaccination alone is sufficient in curing

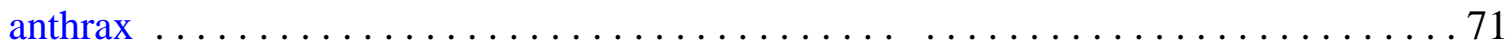

3.5 The clear separation of positive and negative outcomes in terms of tissue dysfunction in a randomized clinical trial. . . . . . . . . . . . . . . . . . . . . . . . . . 76

4.1 Threshold of extinction for various rates of decay - as predicted by the Master equation and Gillespie simulations . . . . . . . . . . . . . . . . . . . . 84

4.2 Time of extinction for decay process with rate $\mathrm{k}(\mathrm{t})=1$ using the various methods.. . 87

4.3 Threshold from Gillespie simulation of 2-d models with varying parameters . . . . 92 


\subsection{INTRODUCTION}

\subsection{THE PROBLEM OF SYSTEMIC INFLAMMATION}

The initial response of the body to an infection or to trauma is called the acute inflammatory response. This is non-specific and is the first line of defense against danger. It consists of a coordinated systemic and local mobilization of immune, endocrine and neurological mediators (Janeway \& Travers, 1997). In a healthy response, inflammation is activated, clears the pathogen (in case of an infectious insult), begins a repair process and abates. However, an improper immune response could result in prolonged uncontrolled systemic inflammation that could lead to multiple organ dysfunction and death. In the case of an infectious insult, this condition is known as "sepsis" and is the most prevalent cause of mortality in Intensive Care Units (ICUs) (Tilney et al., 1973; Eiseman et al., 1977). Severe sepsis is a common and frequently fatal condition with an estimated 750,000 cases annually (1991) in the United States alone (Angus et al., 2001). Although much has been learnt about the molecular and physiologic pathways of acute inflammation, this knowledge has not led to many effective therapies (Cross \& Opal, 2003).

\subsubsection{Acute inflammation}

The purpose of the acute inflammatory response is to clear the invading infectious agent, dead and damaged tissue and to restore homeostasis, the normal state of the body. It often starts off as a local response to the threat and if unresolved, spills over to result in systemic inflammation. (Bone, 1996a).

Physiologically, acute inflammation consists of a web of interacting cells and mediators. 
Phagocytic cells that engulf and kill bacteria, signaling proteins that can up- or downregulate inflammation (pro- and anti- inflammatory cytokines (signaling proteins)), effector molecules, coagulatory mediators all play a role in this process. The process of inflammation can itself cause tissue damage, hypotension and hypoperfusion of major organs. These also contribute to exacerbating inflammation. Dysregulated systemic inflammation could thus result in excessive tissue damage, hypotension and could eventually lead to multiple organ failure and death.

Inflammation has traditionally been associated with redness, edema, fever and malaise. The clinical symptoms of systemic inflammation have been characterized in order to identify and treat patients effectively. Two or more of the symptoms such as fever $\left(>38^{\circ} \mathrm{C}\right)$ or hypothermia $\left(<36^{\circ} \mathrm{C}\right)$, hypotension $(<30 \mathrm{~mm} \mathrm{Hg})$, increased heart rate etc. are diagnostic of the Systemic Inflammatory Response Syndrome or SIRS (Bone et al., 1992). These definitions have also been updated to reflect changes in the understanding of the process (Levy et al., 2003). Systemic inflammation that arises from infectious causes is called sepsis and sepsis accompanied by organ dysfunction is known as severe sepsis. Shock is defined as persistent hypotension despite fluid resuscitation.

Systemic inflammation and the ensuing morbidities were thought to be the result of an excessive pro-inflammatory response. Several immuno-modulatory therapies targeted the major pro-inflammatory players but were found to be ineffective. Currently, it is acknowledged that anti-inflammatory mediators also play an important role in the pathogenesis of inflammation and the imbalance between these processes contributes to the condition (Bone, 1996b).

The pathways of systemic inflammation and the ensuing clinical symptoms are virtually indistinguishable in the cases of varying instigators such as bacteria, viruses, tissue trauma, hemorrhage or more than one of the above insults. Thus the ultimate cause of death is thought to be the improper immune response rather than the original instigator (Alberti et al., 2002). Moreover, the common clinical symptoms defined above also reflect more than one underlying physiologic state - e.g. recurrent infection, an inadequate response that leads to uncleared infection or an exuberant response that leads to cleared infection but unalleivated symptoms - could all be clinically classified as SIRS. 
Systemic inflammation and the ensuing morbidities are very much a product of modern medicine. Before the discovery of advanced resuscitation techniques, patients could not be kept alive long enough for the condition to fully unfold. Patients often died from blood loss and severe infections before uncontrolled inflammation arose. With the advent and improvement of antibiotics and organ support therapy, the condition has become increasingly relevant (Bone, 1996a). The incidence of systemic inflammation is also expected to increase with further advancement of medical technology and the increase in the susceptible population - such as the elderly and HIV infected patients (Angus et al., 2001).

\subsubsection{Pathogenesis of systemic inflammation}

In this section the processes and mediators of the acute inflammatory response to a bacterial infection are described briefly (see for example, (Janeway \& Travers, 1997)). The growing bacteria and sterile bacterial products (such as its cell wall, toxins released, bacterial coat) activate the circulating white blood cells or phagocytic cells (neutrophils and macrophages). They are "chemo-attracted" to the site of the infection and transform from "resting" cells to "activated" cells. Phagocytic cells in their activated form can engulf and destroy bacteria and damaged tissue. They also release cytotoxic chemicals which can oxidize and kill bacteria.

The activation and chemo-attraction of the phagocytic cells stimulates the production of pro-inflammatory cytokines such as TNF- $\alpha$ and IL-1. (Cytokines are proteins secreted by certain cells that act as inter-cellular mediators.) These cytokines have a rapid initial burst and are found to be cleared within the first few hours in animal models. Their production is shut down by rapidly acting anti-inflammatory cytokines such as IL-10 which are released in response to them (Bone, 1996b; Janeway \& Travers, 1997). TNF- $\alpha$ and IL-1 promote recruitment and activation of phagocytes and also result in the second wave of slower cytokines. These include the pro-inflammatory cytokines such as IL-6 and anti-inflammatory cytokines such as TGF- $\beta$. It is important to note that even if the major functions of cytokines are delineated in-vitro, they are often pleiotropic and their systemic effects might be different than the determined local effects.

These inflammatory processes result in the release of cytotoxic substances, such as super- 
oxide $\left(\mathrm{O}_{2}^{--}\right)$and Nitric Oxide $(\mathrm{NO})$, that aim to clear bacteria and dead tissue (Jaeschke \& Smith, 1997). It is postulated that the overwhelming release of these causes damage to healthy tissues as well. The endothelial cells are thought to play a major role in the pathogenesis of shock as they exacerbate inflammation and hypotension. Several other cascades such as the complements and coagulatory mediators are activated in the process of acute inflammation. It is hypothesized that the dysfunction in coagulation which results in hypotension and hypoperfusion of major organs may contribute to death (Bone, 1996a). The major mediators of the coagulation cascade are tissue factor, thrombin and activated protein-C. Tissue factor is stimulated by bacterial toxins and IL-6 and causes the release of thrombin, which is pro-inflammatory and contributes to tissue damage. Thrombin then releases activated protein- $\mathrm{C}$ which is essentially anti-inflammatory and down-regulates the coagulation cascade. The only FDA approved therapy for sepsis is Drotrecogin alpha which adds activated protein-C to the body (Bernard et al., 2001).

In the case of a healthy outcome, inflammation results in the resolution of the original insults and restoration of homeostasis. However, the processes of inflammation which cause tissue damage, hypotension and hypoperfusion of major organs could lead to progressive failure of major organs and eventually death. In fact, failure of the central nervous system, lung and kidney failure and cardio-vascular shock are common causes of ultimate mortality in ICUs (Alberti et al., 2002).

\subsubsection{Therapies for systemic inflammation}

The process of inflammation thus enhances and controls itself through a complex series of redundant and pleiotropic systems which are delicately balanced. Current strategies in ICUs are mainly supportive and include fluid resuscitation, vasopressors, blood transfusion and mechanical ventilation (Vincent, 2002).

The search for the potential immune mediators to be modulated for alleviating the sepsis syndrome has been ongoing for several years. Potential targets have included TNF- $\alpha$, IL1, endotoxin and most recently activated protein-C. Of these, the sole target approved as therapy for severe sepsis is activated protein-C, which reduced mortality by $6 \%$ compared 
with controls in clinical trials (Bernard et al., 2001).

Another major problem in tackling sepsis may lie in diagnosing the condition. In the clinical setting, inflammatory states are defined by symptoms and few biological markers (Bone et al., 1992). For example, Systemic Inflammatory Response Syndrome (SIRS) is a condition characterized by elevated respiratory and heart rates, fever, and an abnormal white blood cell count. The severity of sepsis is based on the presence of circulatory shock (low blood perfusion) and organ failure (ACCP/SCC, 1992; Muckart \& Bhagwanjee, 1997). Similarly, treatment and support of these critically ill patients is largely based on clinical signs and a few biochemical and hematological parameters.

The inflammatory response to an insult varies depending on several factors such as age, co-existing morbidities, gender and genetic polymorphisms. The profile of cytokines in addition, depends on the time elapsed from the onset of infection. For the same insult in different individuals, the response might be a healthy outcome and return to homeostasis, an insufficient response which suppresses but does not clear infection or overwhelming inflammation and shock. The clinical symptoms for the negative outcomes might coincide but require diverse treatment strategies. It is also possible that at different time points in the course of disease, the same individual might be hyper-inflamed or immuno-suppressed. Therefore at what time and to whom the therapy is administered are as important as what mediator is being modulated.

Given the complexity of acute inflammation and the heterogeneity in its manifestation in a population, it is highly unlikely that targeting a single mediator would prove to be an effective strategy It is widely accepted that targeting a combination of mediators is likely to be more successful (Bone, 1993; Cross \& Opal, 2003). Also, different treatment strategies might be required depending on the individual response to the insult.

\subsection{MOTIVATION AND OUTLINE OF THE THESIS}

The acute inflammatory response thus consists of a complex sequence of events that could result in systemic inflammation and death. Despite a wealth of knowledge about the individual processes, treatment strategies are mostly supportive and effective targets for immune- 
modulation remain elusive. One reason for the lack of effective treatments against sepsis may be that the complex nature of the inflammatory response renders the effect of targeting isolated components of inflammation difficult to predict. The need for a systems based theoretical understanding of the sepsis syndrome has been emphasized (Tjardes \& Neugebauer, 2002; Buchman et al., 2001) and such an understanding could be provided by mathematical modeling.

Even simple dynamical models can capture essential features of the system and provide intuition about its design. But predictions from such simple models are often too generic to be useful to biologists. The strategy used in this thesis, is to study models of varying degrees of complexity, each limited to answering a specific set of questions. The smaller models provide qualitative understanding of the dynamics and also facilitate in understanding the larger, more complex models. Such a complex model capturing the behavior of several specific immune mediators has also been constructed. These models and the common dynamics conserved among them are presented in Chapter 2.

The common dynamics and paths to "health" and "shock" are highlighted using a simple Lotka-Volterra type 2-dimensional predator-prey model with a positive feed-back term in the predator equation. This model is then expanded to 3 dimensions by adding another equation to model the feed-back. The terms in the 3-d model are $p$, a growing pathogen, $m$, the early immune response (predator) and $l$, the late immune response which forms a positive feed-back loop with $m$. This model expands on the dynamics of the 2-d model and simulations and bifurcation analyses reveal health, shock and additional negative outcomes and the parameters that affect these outcomes. The reduced models were constructed to understand the dynamics of the global model, which follows in detail the time-course of several immune mediators and their interactions. This model is constructed based on well established mechanisms of the pathogenesis of the disease and shows the health and the negative outcomes shown by the 3 - $\mathrm{d}$ model. Bifurcation analyses reveal similarities in going from one outcome to the other. These models represent a new systematic understanding of the mechanisms of acute inflammation and the pathways to shock. The important role that later feed-back loops play in the pathogenesis of sepsis is emphasized.

Although the scope for analysis is limited in large models, simulations from them are very 
useful in evaluating physiological hypotheses and for in silico experimentation when data is otherwise scarce. In Chap. 3 two such applications of a biologically detailed model are presented. A model reproducing the time course of immune-mediators in anthrax infection is presented. This is essentially a modification of the model presented in Chap. 2 to account for the specific effects of anthrax bacterium. Simulations predicting the effectiveness of a vaccine therapy are discussed. In this case, the evaluation of such a vaccine in human trials is clearly unethical and predictions from such a model are likely to be very useful. The second application is an implementation of a clinical trial evaluating the efficacy of an anti-TNF antibody as a therapy for sepsis. This therapy was attempted but failed to meet standards of efficacy in a randomized clinical trial (Abraham et al., 1998). As our study shows, several factors can influence the efficacy of a drug such as timing and dosage of therapy and patient population to which the therapy is administered. In an in silico clinical trial, there is complete control and knowledge of the variables involved and this could lead to significant insight. Future trials can also be designed with knowledge gained from such models and could result in more optimal use of resources. (Drs. Gilles Clermont and Yoram Vodovotz were major contributors in the building and understanding of the global model and the ensuing modifications.)

The models discussed above are deterministic ODE models and have limitations in capturing the real stochastic process. In Chap. 4, the effect of fluctuations at low concentrations is examined. In a deterministic equation, decaying populations cannot become extinct in finite time. Chapter 4 attempts to use a stochastic description of the processes when the population numbers are small and calculate a threshold of extinction, below which the population may be considered to be extinct. The strategy used is to predict an average time to extinction from a simple one dimensional population decay and examine its usefulness in a more complicated scenario of interacting populations (such as a predator-prey model). Several other techniques have been used to understand extinction in deterministic models and Chap. 4 attempts to provide yet another approach to study this problem. 


\subsection{DYNAMICS OF ACUTE INFLAMMATION - 3 MODELS OF INCREASING COMPLEXITY}

This chapter attempts to understand the dynamics of the acute inflammatory response to infection by using ODEs and the techniques of dynamical systems. Three models of increasing complexity are presented. All the models obey mass-action kinetics in well-mixed volumes. The simplest is a 2-dimensional model that captures only the essential bi-stability between "healthy" and "septic" states and is followed by a 3-dimensional model that captures the bistability and other clinically relevant negative outcomes as well. Bifurcation analysis on the parameters of these models suggests the importance of the various interactions and provides global understanding about the behavior of the system. A larger, more biologically accurate model is also presented in Section 2.3. This model captures the detailed dynamics of various mediators. However, the essential dynamics predicting the pathway to sepsis remains the same in all three models.

Numerical simulations and bifurcation plots in this chapter were generated with XPPAUT (Ermentrout, 2002).

\subsection{SEPSIS IN TWO DIMENSIONS}

A minimal model of sepsis should have the following properties: for some insults, the pathogen is completely eliminated and the system is returns to a "healthy" state and for other insults the system reaches a a shock state where the immune mediators remain elevated and never return to rest. Here, a two dimensional model is constructed that captures these effects. 
In its most simplified description, the immune response to infection can be represented by a Lotka-Volterra type predator-prey model where the prey represents for instance, the bacteria that invade the system and the immune-cells are represented by the predator. This yields the system

$$
\begin{aligned}
\frac{d p}{d t} & =k_{p} p-k_{p m} m p \\
\frac{d m}{d t} & =k_{m p} m p-k_{m} m .
\end{aligned}
$$

This model represents a pathogen growing at rate $k_{p}$ and is ingested when it meets an immune cell at rate $k_{p m} m$. The immune cell is activated on contacting the pathogen at rate $k_{m p} p$ and has a natural deactivation rate $k_{m}$. The behavior of this model can be explained by phase-plane analysis. The fixed points are $p=0, m=0$ which is a saddle point and $p=k_{m} / k_{m p}, m=k_{p} / k_{p m}$ which is a center. Irrespective of the parameters (as long as they are all positive), any initial condition results in an oscillation around the center. Hence this model cannot capture the dynamics of sepsis.

However, replacing (2.2) by

$$
\frac{d m}{d t}=k_{m p} m p-k_{m} m+k_{f} m^{2} .
$$

results in a minimal model. This additional positive feedback introduces an additional fixed point $p=0, m=k_{m} / k_{f}$ which is a saddle if $k_{f}<\left(k_{p m} k_{m}\right) / k_{p}$ and an unstable node otherwise. The center of the previous system $(2.1,2.2)$ becomes $p=\left(1 / k_{m p}\right)\left(k_{f} k_{p} / k_{p m}\right), m=k_{p} / k_{p m}$ and is an unstable spiral (as long as $0<k_{f}<\left(k_{p m} k_{m}\right) / k_{p}$. See Appendix 2.5).

The phase-plane of this model with two sample trajectories is shown in Figure 2.1. One of the trajectories loops around the unstable spiral point and remains close to $p=0$ before spiraling out to $m=\infty$ and the other trajectory goes directly out to $m=\infty$ without spiraling. The latter is interpreted as the septic trajectory where immune mediators remain elevated. The former is interpreted as healthy behavior. This is because when $p$ gets small enough, the approximation of $p$ as a continuous variable breaks down. In this regime, the discreteness of $p$ is important and when $p$ falls below a value representing a single pathogen particle it can be considered to be eliminated. A stochastic model would be the correct description for small particle numbers. For ODE models, $p$ can never be reduced to zero 
in a finite time. Thus, for the models in this chapter, unstable spirals that take $p$ close to 0 are interpreted as healthy behavior. We propose that there is an effective threshold representing a single pathogen particle below which, on average, the pathogen population can be considered to be eliminated and this is discussed in detail in Chapter 4 .

Thus, a positive feed-back in the $m$ equation ensures the bi-stable behavior that we are trying to capture. For large positive feed-back $\left(k_{f} \geq\left(k_{p m} k_{m}\right) / k_{p}\right)$, the unstable spiral is no longer in the positive quadrant and no further oscillations are possible. Then all initial conditions run away to $m=\infty$ or sepsis is the only possible outcome of the system. The positive feed-back also ensures the complete elimination of the pathogen and avoids the Lotka-Volterra oscillations seen in the system $(2.1,2.2)$ which do not (in general) take $p$ close to 0 . The claim made is that the pro-inflammatory positive feed-back is the crucial feature of the acute inflammatory response that ensures elimination of pathogen and the strength of this feed-back controls the predisposition of a system to sepsis. This very feature is observed in the next two models as well.

Realistically, the pathogen is not expected to grow in an uncontrolled fashion in the absence of immune cells. A competition for resources limits the growth and Equation (2.1) is changed to reflect that.

$$
\frac{d p}{d t}=k_{p} p-k_{p m} m p-k_{s} p^{2}
$$

Now, the model system $(2.4,2.3)$ has an additional fixed point $p=k_{p} / k_{s}, m=0$ which is stable when $k_{m}>\left(k_{m p} k_{p}\right) / k_{s}$ and a saddle otherwise (see Appendix 2.5). The unstable spiral of the previous model is changed to a slightly different location, $p=\left(k_{m}-k_{f} m^{*}\right) / k_{m p}, m^{*}=$ $\left(k_{p} k_{m p}-k_{m} k_{s}\right) /\left(k_{m p} k_{p m}-k_{f} k_{s}\right)$, but its instability still depends only on $k_{f}$.

The above analysis is motivated by reasonable biological assumptions and simplicity in modeling. An interesting aside is that the simple predator-prey model $(2.1,2.2)$ can also be modified by adding a positive feed-back term to the $p$ equation instead of logistic saturation:

$$
\frac{d p}{d t}=k_{p} p-k_{p m} m p+\theta p^{2}
$$

In this system $(2.5,2.2)$, the only fixed point in the positive quadrant is $p=k_{m} / k_{m p}, m=$ $\left(1 / k_{p m}\right)\left(\theta k_{m} / k_{m p}+k_{p}\right)$ which is an unstable spiral as long as $\theta>0$. In such a model, with pathogen exhibiting extra growth on collisions (which is not usually observed biologically) 
and no feed back in the immune system, all initial conditions lead to unstable spirals and thus the pathogen is always eliminated and there is no risk of sepsis. However, in addition to being implausible, pathogens with other growth characteristics would not be eliminated. Appendix 2.5 discusses the behavior of the two dimensional model in greater detail.

In the above modeling scheme, unstable spirals in the phase-plane are interpreted as healthy behavior. A model where healthy behavior is instead represented by a stable fixed point can also be constructed. For instance,

$$
\begin{aligned}
\frac{d p}{d t} & =k_{p} p-k_{p m} m p-k_{1}\left(p-p_{0}\right) p+k_{2}\left(p-p_{s}\right)^{3} p \\
\frac{d m}{d t} & =k_{m p} m p-k_{m} m+k_{3}\left(m-m_{0}\right) m-k_{4}\left(m-m_{s}\right)^{3} m
\end{aligned}
$$

is a system where $\left(p_{0}, m_{0}\right)$ could be the stable healthy fixed point and $\left(p_{s}, m_{s}\right)$ the stable septic fixed point. This model requires the pathogen to have a very specific (and unrealistic growth profile). The model that we have presented has simpler biological interpretations and does not demand any special properties from pathogen other than what is considered to be its essential behavior.

\subsection{A 3-D MODEL OF ACUTE INFLAMMATION}

In this section, a simple 3-dimensional model of the inflammatory response to infection is discussed. This model includes a third variable to provide a saturating positive feed-back to the early immune response replacing the $k_{f} m^{2}$ term in the 2-d model. Although the essential dynamics do not change, an independent variable modeling late immune response is a better approximation of reality. Positive feed-back mechanisms that enhance the pro-inflammatory environment (such as phagocytic cells activated by immune-mediators (macrophages, neutrophils activated by TNF- $\alpha, \mathrm{IL}-1)$; early immune-mediators promoted by later mediators (TNF- $\alpha$ promoted by HMGB-1 etc.) are well documented processes of the acute inflammatory response (Janeway \& Travers, 1997; Bone, 1996a; Wang et al., 2001). The 3-d model also exhibits various states which can be more readily identified with clinically relevant behavior. The diverse scenarios that arise are clearly defined and the ambiguous terminology 


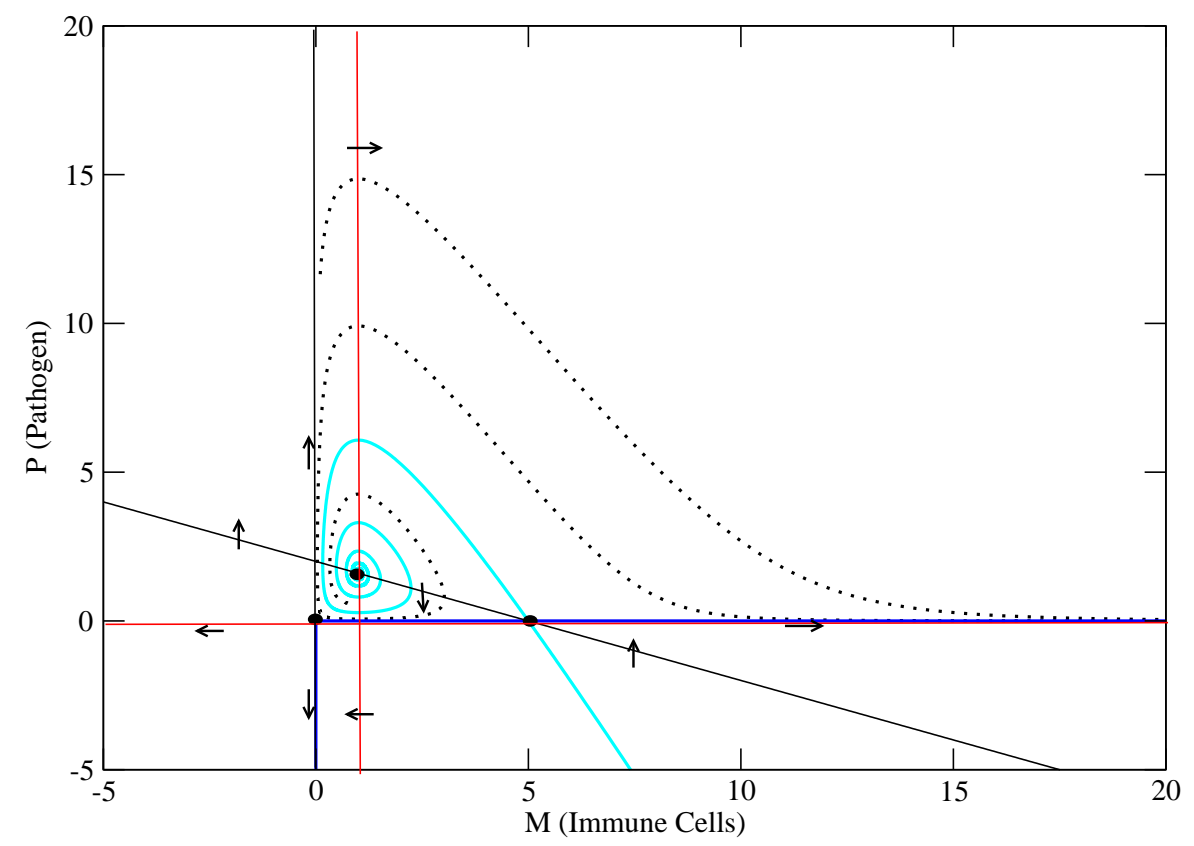

Figure 2.1: The phase-plane for the 2-d model (2.1,2.3) showing "healthy" and "shock" trajectories and the stable manifold that separates them. The red curves (marked with horizontal arrows) correspond to conditions when $\frac{d p}{d t}=0\left(p=0\right.$ and $\left.m=k_{p} / k_{p m}\right)$ and the black curves (marked with vertical arrows) correspond to conditions when $\frac{d m}{d t}=0$ ( $m=0$ and $\left.p=\left(k_{m}-k_{f} m\right) / k_{m p}\right)$. The fixed points are at the intersections of black and red curves and the direction of flow along a curve reverses at fixed points. The phase-space for a typical set $\left(k_{p}=1.0, k_{p m}=1.0, k_{m p}=0.5, k_{f}=0.2, k_{m}=1.0\right)$ of parameters is shown. The stable and unstable manifolds are shown in thicker lines. A typical healthy trajectory and sepsis trajectory shown in dotted lines. 
of "sepsis" is avoided in this section. The model can show the following behavior: a healthy response where pathogen is cleared and the body returns to homeostasis, recurrent infection where inflammation is inadequate and the pathogen cannot be completely eliminated, persistent infectious inflammation where the pathogen levels and inflammation are high, persistent non-infectious inflammation where pathogen is cleared but inflammation persists and severe immuno-deficiency where pathogen has grown to saturation but the inflammatory response is very low. The model suggests that "sepsis" is a multifaceted disease and narrowly targeted interventions are unlikely to succeed.

The unstable spiraling trajectory which represents healthy behavior in the 2-d model is also present here and the spiraling trajectories are formed through sub-critical Hopf bifurcations. The runaway persistent inflammation trajectory is controlled by a saturating term and hence persistent inflammation is a stable fixed point in this model. Additionally, immuno-suppressed and recurrent infections are exhibited in the 3-d model which have no clear analog in the 2-d model. Through a systematic analysis of the influence of parameters, more detailed conclusions can be drawn about the acute inflammatory response.

In acute inflammation, an infectious pathogen triggers early pro-inflammatory responders which attempt to kill the pathogen. The early inflammatory mediators then activate later inflammatory mediators which can further excite the early mediators. This is the basis of the model which consists of three variables: 1) a pathogen $p$, which is an instigator of the innate immune response; 2) an early pro-inflammatory mediator $m$, which can be thought of as representing the combined effects of immune cells such as macrophages and neutrophils together with early pro-inflammatory mediators such as TNF- $\alpha$ and IL-1; 3) a late pro-inflammatory mediator $l$ which represents a late pro-inflammatory feedback. This is a combined effect of cytokines such as IL-6, HMGB-1 and stimulatory effects of tissue damage and dysfunction.

The model is as follows:

$$
\begin{aligned}
\frac{d p}{d t} & =k_{p} p(1-p)-k_{p m} m p \\
\frac{d m}{d t} & =\left(k_{m p} p+l\right) m(1-m)-m \\
\frac{d l}{d t} & =k_{l m} f(m)-k_{l} l
\end{aligned}
$$


where

$$
f(m)=1+\tanh \left(\frac{m-\theta}{w}\right)
$$

$\theta$ is an activation threshold and $w$ is an activation width. All the variables and parameters are non-negative. The pathogen $p$ obeys logistic growth and is killed when it interacts with $m$. The presence of $p$ or $l$ and $m$ will stimulate the growth of $m$ which also has an intrinsic death rate. This growth saturates as $m$ increases towards unity mimicking the effects of cell depletion and anti-inflammatory cytokines. The late mediator $l$ is recruited by $m$ through a sigmoidal coupling function (2.11) and is cleared with the rate of $k_{l}$. The parameters that determine strength of the $l m$ interaction term, the saturating parameters in the $p$ and $m$ equation and the decay rate of $m$ are used to scale $p, m, l$ and $t$. In summary, the dynamics obey predator-prey dynamics with a delayed response.

\subsubsection{Dynamics of the model - Numerical examples}

The model (2.8-2.10) exhibits behavior reminiscent of what is observed in clinical settings. Given an initial condition of $p>0, m>0$ and $l>0, p$ grows, inducing $m$ and $l$ to grow and attempt to eliminate $p$. Depending on initial conditions and parameters, the ensuing orbits either approach stable fixed points or undergo oscillations, each having a physiological interpretation. In (2.9) a non-zero positive initial value for $m$ is necessary to generate an inflammatory response. The background level of late mediators is given by $l^{0}=\left(k_{l m} / k_{l}\right) f(0)$ and is non-zero for the present choice of parameters. Both these properties are consequences of the particular choice for the form of the model and not essential for the qualitative results found.

As discussed in the previous section, the unstable spiral is interpreted as healthy behavior by introducing a threshold for pathogen level. When $p$ falls below this threshold, it is considered to be completely cleared. Numerical examples are considered for various values of $k_{p m}, k_{m p}$, and $k_{l m}$. In Section 2.2.2, it is shown that these are natural bifurcation parameters of the system. The other parameters are fixed at $k_{p g}=3, k_{l}=1, \theta=1$ and $w=0.5$. The effects of varying the parameters $\theta$ and $w$ are shown in Appendix 2.6.2.1.

A healthy response to infection as seen in Figure 2.2 corresponds to an orbit that spi- 
rals outwards so that $p$ falls below threshold during the oscillation. The pathogen is then completely cleared which allows the inflammatory response to relax back to rest.

In Figure 2.3, the same parameters are used but the initial pathogen load is higher so that instead of returning to background levels, the inflammatory mediators are over-excited and remain elevated. This is related to a state of persistent non-infectious inflammation where even though the pathogen is cleared, the inflammatory response does not abate. In the model, this state is a fixed point but in a real organism if this condition continued, it would eventually lead to multiple organ failure and death (Reyes et al., 1999).

Starting again from a healthy situation, if the pathogen susceptibility to the host's defenses $\left(k_{p} m\right)$ is decreased, persistent infectious inflammation is observed where the inflammatory response is high but the pathogen still cannot be cleared as seen in Figure 2.4. This condition is equated with a severe septic state where both infection and inflammation are uncontrolled. In this case the damage caused by both the pathogen and inflammation are disrupting body function and if unabated death will result. Patients with systemic inflammation, with and without documented infection, are observed in clinical settings (Alberti et al., 2002).

If the recruitment rate of $l\left(k_{l m}\right)$ is reduced, the healthy response can be turned into one of recurrent infection as seen in Figure 2.5. (Changes in other parameters could also lead to recurrent infection as seen in the next section). In this case, low levels of infection persist indefinitely. This could be likened to infection with tuberculosis, yeast infections or low-grade bacterial infections that persist for long periods of time (Beatty et al., 1994). Although, a host could survive this state for a long duration it may eventually succumb.

Finally, in Figure 2.6, the activation rate of $m$ due to $p\left(k_{m p}\right)$ is very weak, a state of immuno-suppression or immuno-deficiency is observed where the pathogen grows to saturation and does not elicit any response from the body. This could happen if the immune-system had already been compromised by previous infection or trauma and then the organism is re-infected. Opportunistic bacterial and fungal infections have been observed in immunosuppressed patient populations such as HIV infected patients, the elderly and those with organ transplants (Schultz et al., 2001).

These five scenarios are the only possible outcomes in this 3 - $\mathrm{d}$ model. 


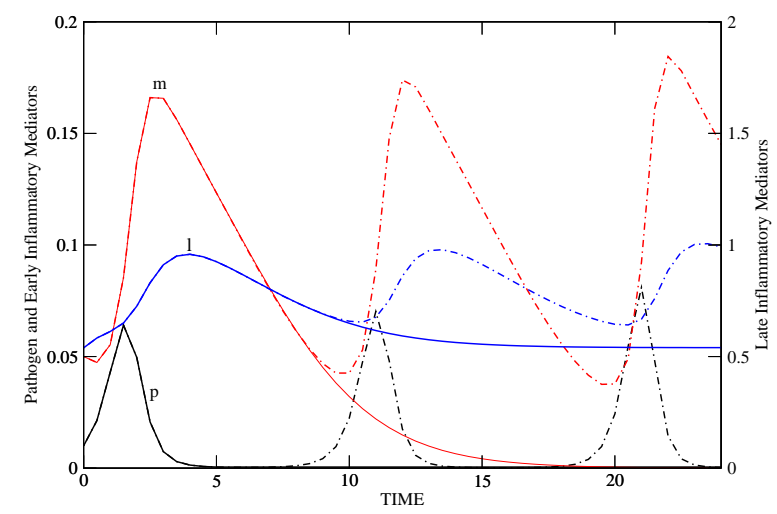

Figure 2.2: Time courses of $p, m$ and $l$ for the healthy response. Orbits with the $p$ elimination threshold (solid line) and without (dotted line) are shown. With the threshold (set at $\left.p_{0}=0.0005\right)$, when $p$ drops below $p_{0}, p$ is set to zero and $m$ and $l$ return to background values. Without the threshold, the orbits spiral outwards. Parameters used are $k_{p m}=30, k_{p}=$ $3, k_{m p}=25, k_{l m}=15, k_{l}=1$. Initial conditions are $p(0)=0.01, m(0)=0.05, l(0)=0.539$.

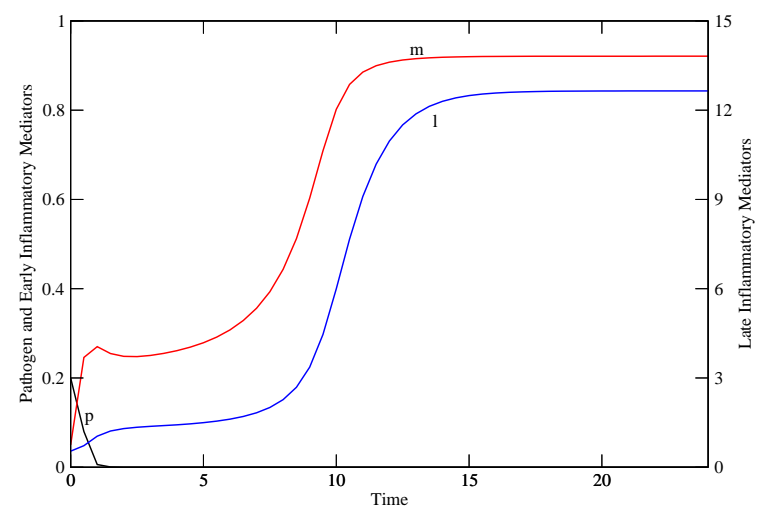

Figure 2.3: In persistent non-infectious inflammation, $p$ is eliminated but $m$ and $l$ remain elevated. Parameters used are $k_{p m}=30, k_{p}=3, k_{m p}=25, k_{l m}=15, k_{l}=1$. Initial conditions are $p(0)=0.2, m(0)=0.05, l(0)=0.539$. 


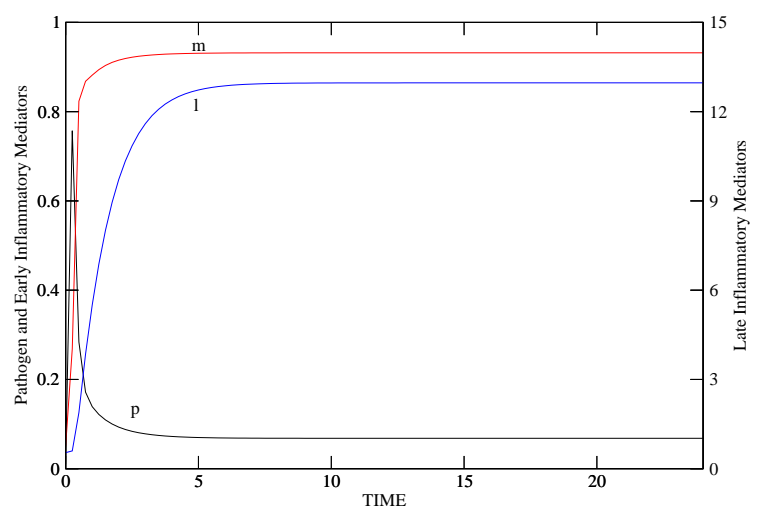

Figure 2.4: In persistent infectious inflammation, $p$ cannot be eliminated and $m$ and $l$ remain elevated (as in severe sepsis). Parameters used are $k_{p m}=3, k_{p}=3, k_{m p}=25, k_{l m}=15, k_{l}=$ 1. Initial conditions are $p(0)=0.01, m(0)=0.05, l(0)=0.539$.

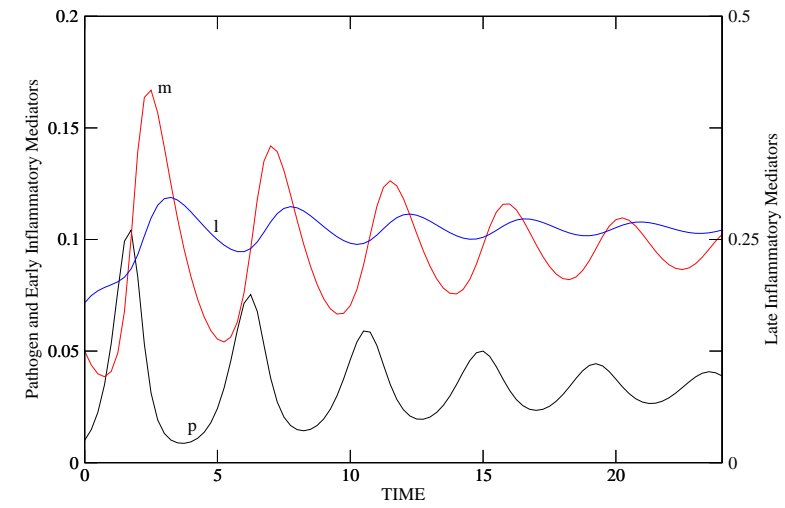

Figure 2.5: In recurrent infection, $p$ is low and $m$ and $l$ remain above background values but not very high (as in a low-grade infection). Parameters used are $k_{p m}=30, k_{p}=3, k_{m p}=$ $25, k_{l m}=5, k_{l}=1$. Initial conditions are $p(0)=0.01, m(0)=0.05, l(0)=0.179$. 


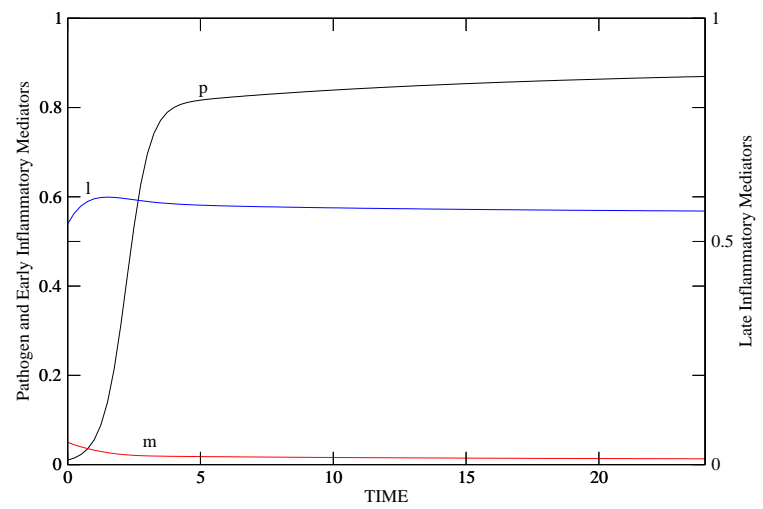

Figure 2.6: In severe immuno-deficiency, $p$ grows to saturation and $m$ and $l$ remain low or absent. Parameters are $k_{p m}=30, k_{p}=3, k_{m p}=0.4, k_{l m}=15, k_{l}=1, p(0)=0.01, m(0)=$ $0.05, l(0)=0.539$.

\subsubsection{Dynamics of the model - Fixed Points and Bifurcations}

These various regimes and transitions are best understood by examining the fixed points and associated bifurcations of the model (2.8-2.10). The fixed points satisfy the following conditions:

$$
\begin{aligned}
0 & =p\left[p-1+\left(\frac{k_{p m}}{k_{p}}\right) m\right] \\
0 & =m\left[m-1+\left(\frac{1}{k_{m p} p+l}\right)\right] \\
l & =\frac{k_{l m}}{k_{l}} f(m)
\end{aligned}
$$

Substituting (2.14) into (2.13) and rearranging gives

$$
0=m\left[p-\frac{1}{k_{m p}}\left(\frac{1}{1-m}-\frac{k_{l m}}{k_{l}} f(m)\right)\right] .
$$

Three natural parameters that appear in the fixed point conditions, (2.12) and (2.15) are considered: $a \equiv k_{p m} / k_{p}, k_{m p}$, and $b \equiv k_{l m} / k_{l}$. These three parameter combinations represent the pathogen susceptibility to $m$ compared to its growth rate (i.e. inverse of the pathogen 


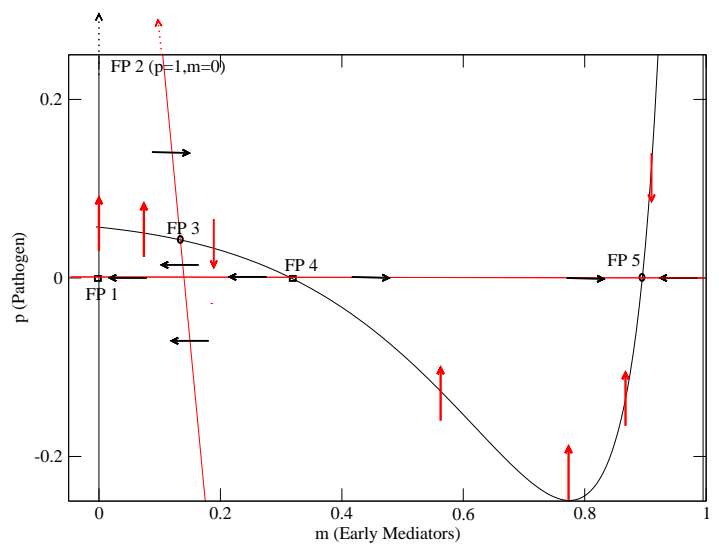

Figure 2.7: Projection of fixed-point conditions onto the $p-m$ plane. Projected directions of field-flow are also marked. The red curves (marked with horizontal arrows) correspond to conditions when $\frac{d p}{d t}=0(p=0$ and $(2.16))$ and the black curves (marked with vertical arrows) correspond to conditions when $\frac{d m}{d t}=0(m=0$ and (2.17)). The fixed points are at the intersections of black and red curves and the direction of flow along a curve reverses at fixed points. The projection of the phase-space for a typical set of parameters is shown. FP 1 and FP 4 are saddles. FP 3 is a spiral which is stable or unstable based on the actual values of the parameters. FP 5 is a stable fixed point. FP 2 (not shown) is also a saddle in this case. 
virulence), the activation rate of early responders $m$ due to $p$, and the effective recruitment rate of $l$ due to $m$, respectively.

The intersections of conditions (2.12) and (2.15) give the fixed points of the system. The dependence of the fixed points with the parameters is best observed as a projection in the $p-m$ plane as shown in Figure 2.7 where the fixed point conditions (2.12) and (2.15) are plotted. Lines $p=0$ and $m=0$ are unaffected by changes in parameters. The line

$$
p=1-a m
$$

is affected only by $a$ and sweeps across the $p-m$ plane as $a$ is decreased. The curve

$$
p=\frac{1}{k_{m p}}\left(\frac{1}{1-m}-b f(m)\right)
$$

is affected only by the immune parameters $b$ and $k_{m p}$. It drops below $p=0$ as $b$ is increased. Changing $k_{m p}$ affects the height and angle at which (2.17) intersects (2.16). Note that the formation of FP 3 which undergoes Hopf bifurcations in Figure 2.7 is reminiscent of the oscillations in the 2-d phase-plane in Figure 2.1. The $p=0$ and $m=0$ nullclines ensure that all trajectories which start in the positive quadrant stay in it.

There are five fixed points which have been labeled from FP 1 to FP 5. FP 1 is given by $p=0, m=0$, and $l=\left(k_{l m} / k_{l}\right) f(0)$. This fixed point is always unstable because the pathogen is growing. FP 2 is given by $p=1, m=0, l=\left(k_{l m} / k_{l}\right) f(0)$ and may be interpreted as a severely immuno-deficient state. Here the pathogen has grown to saturation, but there is no early immune response and the late response remains at the background level. This point is stable when the early immune response is very weak as shown in Appendix 2.6.1.2.

FP 4 and FP 5 arise from a saddle node bifurcation when $p=0$ in (2.17). This can be achieved by increasing $b$ from zero as seen in the bifurcation plot in Figure 2.10. FP 4 is always unstable and never represents any physiologically relevant scenario. When stable, FP 5 represents the persistent non-infectious inflammation fixed point. FP 3 is given by the intersection of the line (2.16) with the curve (2.17). FP 3 could represent healthy, recurrent infection or persistent infectious inflammation states, depending on parameter values. Below, the three parameter combinations $a, k_{m p}$ and $b$ are varied and the bifurcation plots are examined. 
In the healthy scenario, $a$ is large enough so that FP 3 is an unstable spiral and FP 5 is the only stable node $\left(k_{p m}=20\right.$ in Figure 2.8). In this case, initial conditions close to FP 3 undergo oscillations that take the pathogen below the elimination threshold and are interpreted as healthy trajectories. A higher pathogen initial condition for the same set of parameters takes the trajectories directly to FP 5 and this behavior is interpreted as persistent non-infectious inflammation. This is similar to the trajectories seen in the phase-plane of the 2-d model in Figure 2.1. When $a$ is decreased, the slope of line (2.16) decreases and it sweeps through FP 4 rendering it unstable through a transcritical bifurcation. However, $p$ is negative and thus FP 3 becomes unphysical $\left(k_{p m}=10\right.$ in Figure 2.8). Hence FP 5 is the only stable fixed point for all initial conditions in this regime. As $a$ is decreased further FP 3 crosses FP 5 and they exchange stability through another transcritical bifurcation. FP 3 then becomes the only stable fixed point and is the global attractor for all initial conditions.

As expected, starting from a healthy scenario, the outcome of the system becomes more and more severe with decreasing pathogen susceptibility (i.e. increasing effective pathogen virulence). If we were to increase susceptibility $a$ starting from the healthy scenario, FP 3 changes into a stable spiral surrounded by an unstable limit cycle through a subcritical Hopf bifurcation. Now, trajectories within the unstable limit cycle spiral into the stable fixed point FP 3 resulting in recurrent infection and unstable spiral trajectories outside the unstable limit cycle will eventually dip below threshold and be interpreted as healthy. As FP 5 is still the attractor when the initial $p$ or $m$ is very high, this set of parameters could lead to three different outcomes based on the initial conditions $\left(k_{p m}=40\right.$ in Figure 2.8). Increasing $a$ further, increases the radius of the unstable limit cycle until it undergoes a homoclinic bifurcation when it collides with FP 1 . Here FP 3 is a stable spiral and only recurrent infection is supported. The model therefore predicts that pathogen clearance is not possible for a range of high pathogen susceptibility. However, as susceptibility is increased much further, FP 3 gets closer to the origin so that oscillations around it take $p$ below threshold and may be interpreted as healthy.

Varying $k_{m p}$, the strength of the early response, does not affect the stability or position of FP 5, the persistent non-infectious inflammation point. Decreasing $k_{m p}$ takes a healthy state to a state where healthy and recurrent infection co-exist and finally to one of recurrent 
infection only as shown in Figure 2.9. Decreasing $k_{m p}$ below $1-\left(k_{l m} / k_{l}\right) f(0)$ makes FP 3 collide with FP 2 (the severely immuno-deficient state where $p$ grows to saturation and $m$ and $l$ remain at background values). In a transcritical bifurcation, FP 2 becomes the stable fixed point of the system and FP 3 becomes unphysical since $m<0$. Increasing $k_{m p}$ from the healthy value reduces the value of $p$ in FP 3 - this reduces the range of initial conditions for healthy behavior. However, for even very large values of $k_{m p}$, FP 3 is an unstable spiral with complex eigenvalues supporting healthy behavior for initial conditions starting close to FP 3.

Varying the strength of the late response, $b$ affects both FP 3 and FP 5 . As $b$ is decreased, FP 3 undergoes a sub-critical Hopf followed by the homoclinic bifurcation of the unstable limit cycle similar to the above cases (Figure 2.10). FP 4 and FP 5 are created in a saddlenode bifurcation when the curve (2.17) intersects $p=0$ by increasing $b$. However, there is also an upper limit to $b$ beyond which healthy behavior is not supported. The curve (2.17) is below zero when it intersects (2.16) and FP 3 is unphysical. Thus when the late response is too high, persistent non-infectious inflammation is the only possible outcome.

In the preceding, although the behavior of the system has been examined by mostly varying only one parameter at a time, it gives us a picture of the global dynamics of the system. Given the strong nonlinear saturation in the dynamics, orbits can either approach a fixed point, a limit cycle, or a strange attractor. Given that FP 1 and FP 4 are always unstable and FP 2 is stable only when $k_{m p}$ is very low, this leaves FP 3 (or stable limit cycles around it) and FP 5 as the only candidates for global attractors. Ideally however, FP 3 should be an unstable spiral or be surrounded by an unstable limit cycle, so that oscillations around it can be interpreted as healthy behavior.

This gives an explanation for why healthy behavior always entails the risk of uncontrolled inflammation. In order for the pathogen to be cleared, an unstable spiral that can take $p$ below threshold is required. However, if it were not for the (externally imposed) elimination threshold the orbit would eventually end up at some attractor and persistent non-infectious inflammation is the global attractor. Conversely, if the curve (2.17) has not yet intersected $p=0$ to form FP 4 and FP 5 (no persistent inflammation fixed point), FP 3 or a stable limit cycle around it (recurrent infection) is the global attractor. 
There is a possibility that FP 3 could undergo a supercritical Hopf bifurcation (see Appendix 2.6.1.3) which leads to a stable limit cycle around it. However, this is generically interpreted as recurrent infection because fine tuning would be required to ensure that the limit cycle takes $p$ below threshold. Although, not possible in this model, a strange attractor might occur in higher dimensional models. It is unlikely given the large amounts of negative feedback and dissipation in the system. However, even if it were to exist, it may still not lead to a healthy situation as $p$ may never be eliminated or the early and late inflammatory mediators could stay elevated albeit in a chaotic manner.

Physiologically, this implies that in order to completely rid the body of a pathogen, the inflammatory response must respond strongly and remain elevated for a long enough time. If it responds too weakly, then the pathogen persists. If it abates too quickly, then there will be recurrent oscillatory infection. However, if it responds too strongly and too persistently then there is a risk that it will be self-sustaining even after the pathogen is cleared. Thus, there is a trade-off between being able to eliminate pathogens completely and risking non abating inflammation. In Appendix 2.6.2, the parameter ranges where a healthy response is possible are calculated.

In the next section, 2 parameter bifurcation plots are discussed which shed more light on the dynamics of the model.

\subsubsection{2 parameter bifurcations}

The two bifurcations that are crucial in determining the behavior of the system are - the saddle-node bifurcation which results in the creation of the persistent inflammation fixed point and the sub-critical Hopf bifurcation which results in unstable oscillations which are interpreted as healthy.

In this section, bifurcation plots varying the three main parameters, $a, k_{m p}$ and $b\left(k_{l m} / k_{l}\right)$ are examined. The generic saddle-node and Hopf bifurcations are followed by varying two parameters ( $a \& b$ and $k_{m p} \& b$ ) at a time. It is noted that once the saddle-node bifurcation creates the stable persistent inflammation fixed point, for all higher parameter values, the system is susceptible to persistent inflammation. For a parameter range on either side of the 


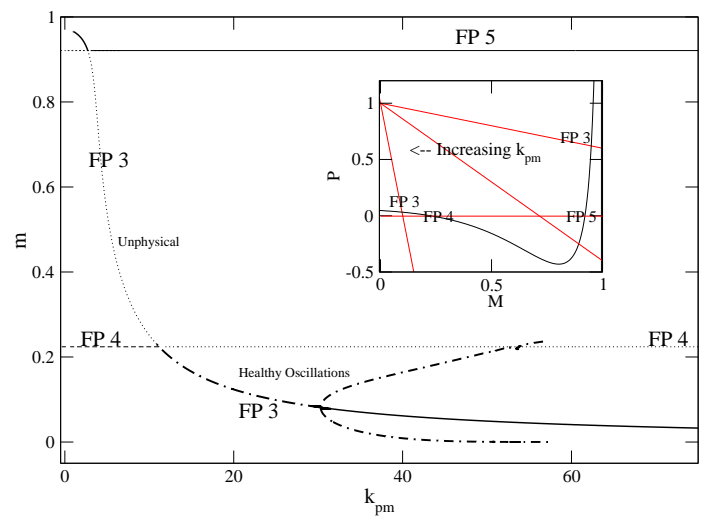

Figure 2.8: Bifurcation plot showing outcome as $k_{p m}$ and hence $a=k_{p m} / k_{p}$ is increased. For low $k_{p m}$, FP 3 interpreted as persistent infectious inflammation is the only fixed point of the system. As $k_{p m}$ is increased to $\approx 2.7, \mathrm{FP} 5$ (persistent infectious inflammation) becomes the attractor of the system. FP 3 becomes unphysical and moves leftward in the $p$ - $m$ plane (see inset). At $k_{p m} \approx 11, \mathrm{FP} 3$ undergoes a transcritical bifurcation with $\mathrm{FP} 4$, becoming physical again. It is an unstable spiral, oscillations around which are interpreted as healthy. On further increasing $k_{p m}$, FP 3 undergoes a subcritical Hopf bifurcation and is surrounded by an unstable limit-cycle, oscillations around which are interpreted as healthy. Trajectories within the limit-cycle, which spiral into FP 3, are interpreted as recurrent infection. Healthy behavior is lost to a homoclinic bifurcation at $k_{p m} \approx 57$. For large $k_{p m}$, FP 3 and FP 5 are bistable (i.e. recurrent infection and persistent non-infectious inflammation are possible outcomes of the system). (Inset : In the $p$ - $m$ plane, line (2.16) sweeps across the plane causing the above bifurcations.) 


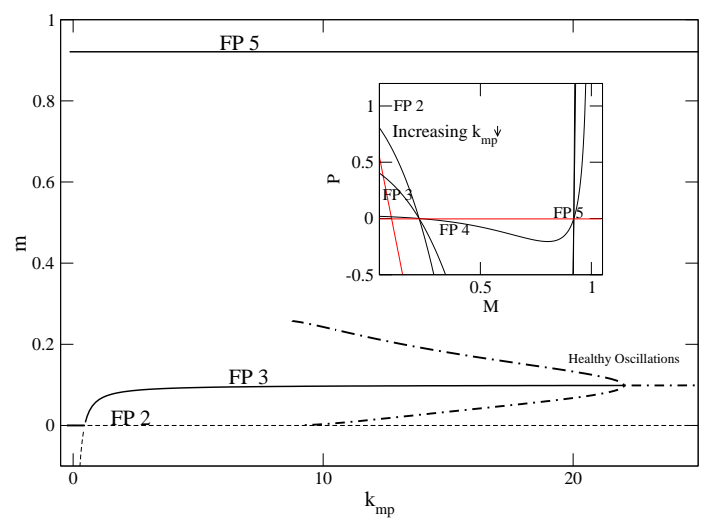

Figure 2.9: Bifurcation plot showing outcome as $k_{m p}$ is increased. For very low $k_{m p}(<0.5)$, FP 2 is stable and is interpreted as the severely immuno-deficient state. As $k_{m p}$ is increased, FP 3 becomes stable through a transcritical bifurcation and recurrent infection is a possible outcome of the system. As $k_{m p}$ is increased further FP 3 undergoes a subcritical Hopf bifurcation $\left(k_{m p} \approx 22\right)$. Oscillations around the unstable limit cycle surrounding FP 3 are interpreted as healthy and are lost through a homoclinic bifurcation $\left(k_{m p} \approx 9\right)$. Trajectories within the limit-cycle spiral into FP 3 and are interpreted as recurrent infection. Beyond the Hopf bifurcation $\left(k_{m p}>22\right)$, FP 3 remains an unstable spiral, up to very large $k_{m p}$ $\left(k_{m p} \approx 2000\right)$ and oscillations around FP 3 are interpreted as healthy. On varying $k_{m p}$, only the position and eigenvalues of FP 3 are changed. FP 5 remains unaffected which is interpreted as persistent infectious inflammation and remains a possible outcome of the system. If curve (2.17) is shallow enough ( $b$ was small enough) so that FP 5 is not yet created, then FP 3 remains the stable global attractor through changes in $k_{m p}$. (Inset : In the $p$ - $m$ plane, FP 3 varies in position and stability as (2.17) varies, causing the above bifurcations. FP 5 remains unaffected.) 


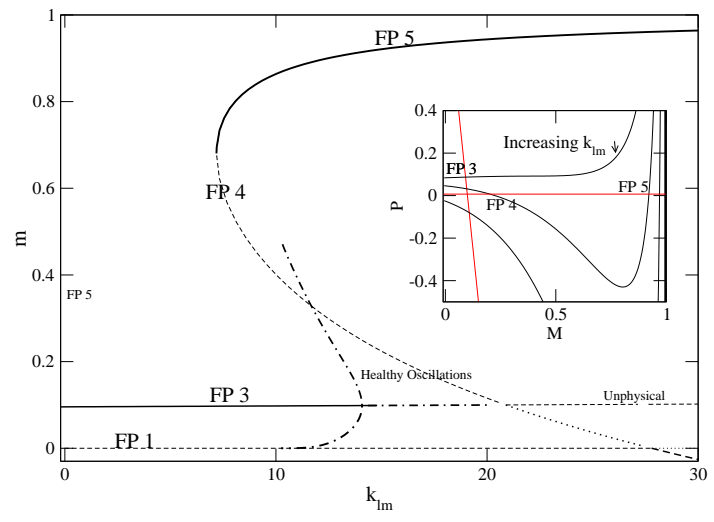

Figure 2.10: Bifurcation plot showing outcome as $k_{l m}$ or $b=k_{l m} / k_{l}$ is increased. For low $k_{l m}$, FP 3 is a stable spiral representing recurrent infection. As $k_{l m}$ is increased, FP 4 and 5 are created through a saddle-node bifurcation at $k_{l m} \approx 7$ and the persistent non-infectious inflammation is now a possible outcome. At $k_{l m} \approx 14$, FP 3 undergoes a subcritical Hopf bifurcation and is surrounded by an unstable limit cycle. The oscillations around the unstable limit cycle are interpreted as healthy behavior and trajectories inside the limit cycle spiral into FP 3 and are interpreted as recurrent infection. Healthy behavior is lost at $k_{l m} \approx 10$ due to a homoclinic bifurcation. Beyond the Hopf bifurcation, healthy oscillations are still supported by FP 3 which is now an unstable spiral. At $k_{l m} \approx 21$, FP 3 meets FP 4 and becomes unphysical through a transcritical bifurcation (as its $p<0$ ). Beyond this point, FP 5 is the only stable attractor and persistent non-infectious inflammation is the only possible outcome of the system. (Inset : In $p$-m plane, (2.17) descends as $k_{l m}$ is increased causing the above bifurcations.) 
sub-critical Hopf line, healthy behavior is supported. A homoclinic bifurcation at one end and a transcritical bifurcation on the other end limit the range of healthy oscillations. Both of these bifurcations are non-generic. This analysis reinforces some of the main conclusions of the previous section. Bifurcation plots varying the parameters of the coupling curve $\theta$ and $w$ are also examined.

In Figs. 2.11, 2.12, the effect of varying the strength of early $\left(k_{m p}\right)$ and pathogen virulence $\left(k_{p m}\right)$ with late response $(b)$, on the saddle-node and Hopf bifurcations is shown. In both cases, the saddle-node bifurcation that results in the creation of the persistent inflammation fixed point is dependent only on the strength of late inflammation (b). The Hopf bifurcation is influenced by all parameters. In Fig. 2.12, the line of Hopf bifurcations continues to the "left" of the saddle-node bifurcations. These Hopf points $\left(k_{l m}<\approx 7\right)$ are super-critical and cannot be generically interpreted as healthy behavior. Thus, we still claim that the risk of persistent inflammation is retained to ensure complete elimination of pathogen.

In the above analyses, the parameters that determine the shape of the coupling curve between $m$ and $l, f(m)$ are kept fixed $(\theta=1$ and $w=0.5)$. These parameters affect both the Hopf and saddle-node bifurcations. Parameter $\theta$ sets the threshold where $l$ is activated by $m$ and parameter $w$ gives the steepness of recruitment. Figure 2.14 shows the coupling curves at various values of $w$ and $\theta$ and the codim- 2 saddle-hopf bifurcation obtained when all other parameters are kept fixed. $w$ and $\theta$ less than 1 result in the maximizing the range of $k_{l m}$ in which healthy behavior is observed as shown in Fig. 2.13.

\subsubsection{Discussion of the $3-\mathrm{d}$ model}

This simple model of acute inflammatory response to infection shows the various negative outcomes that arise from improper inflammatory response. These scenarios of persistent inflammation (with and without infection), immuno-suppression and recurrent infection have all been observed in critically ill patients (Alberti et al., 2002; Bone, 1996a). Vastly different therapeutic strategies are called for to deal with the diverse negative outcomes. Although strict correspondence with clinical reality is difficult to establish because of the simplicity of the model, the model behavior is classified into the same broad categories. 


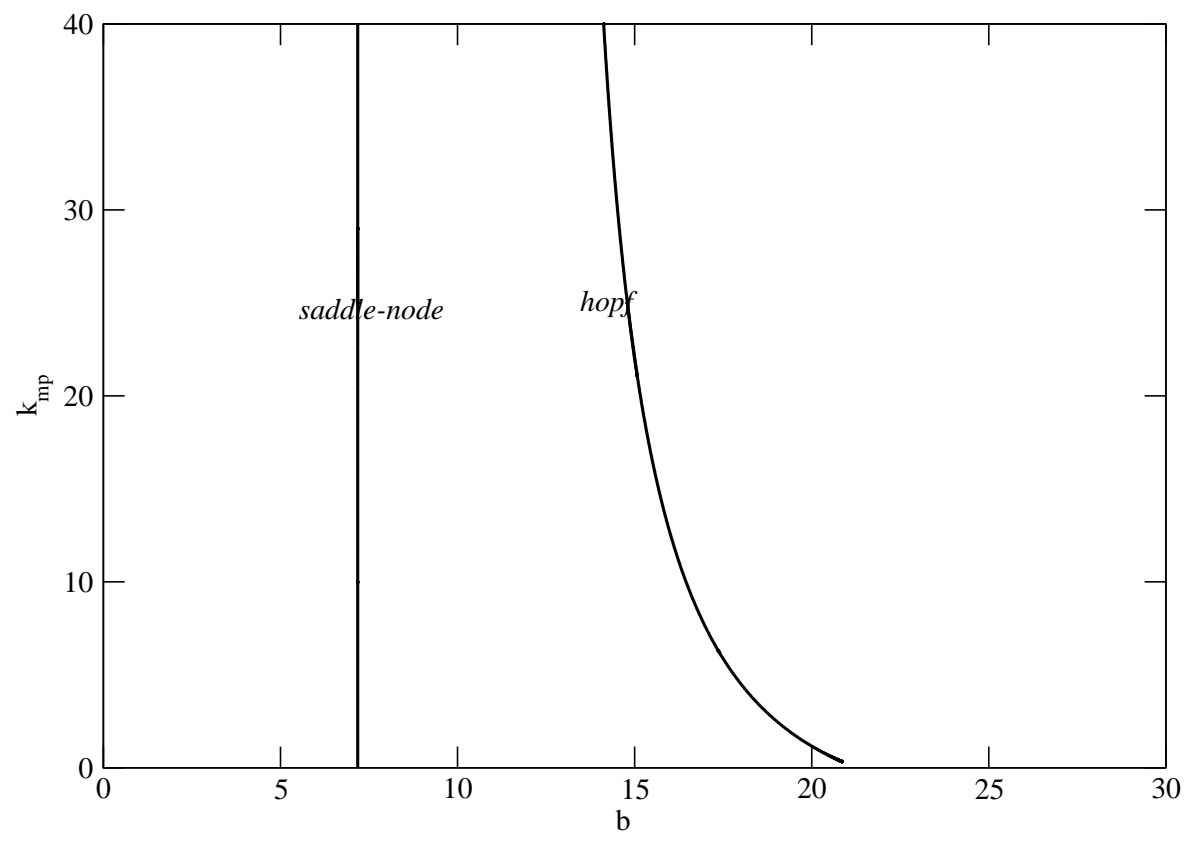

Figure 2.11: A 2 parameter bifurcation plot that varies the strength of early and late inflammatory mediators and follows the saddle-node bifurcation that gives rise to the persistent inflammation fixed point. Only the strength of the late mediators (same $b$, as long as $\theta$ and $w$ remain the same) affects this bifurcation. 


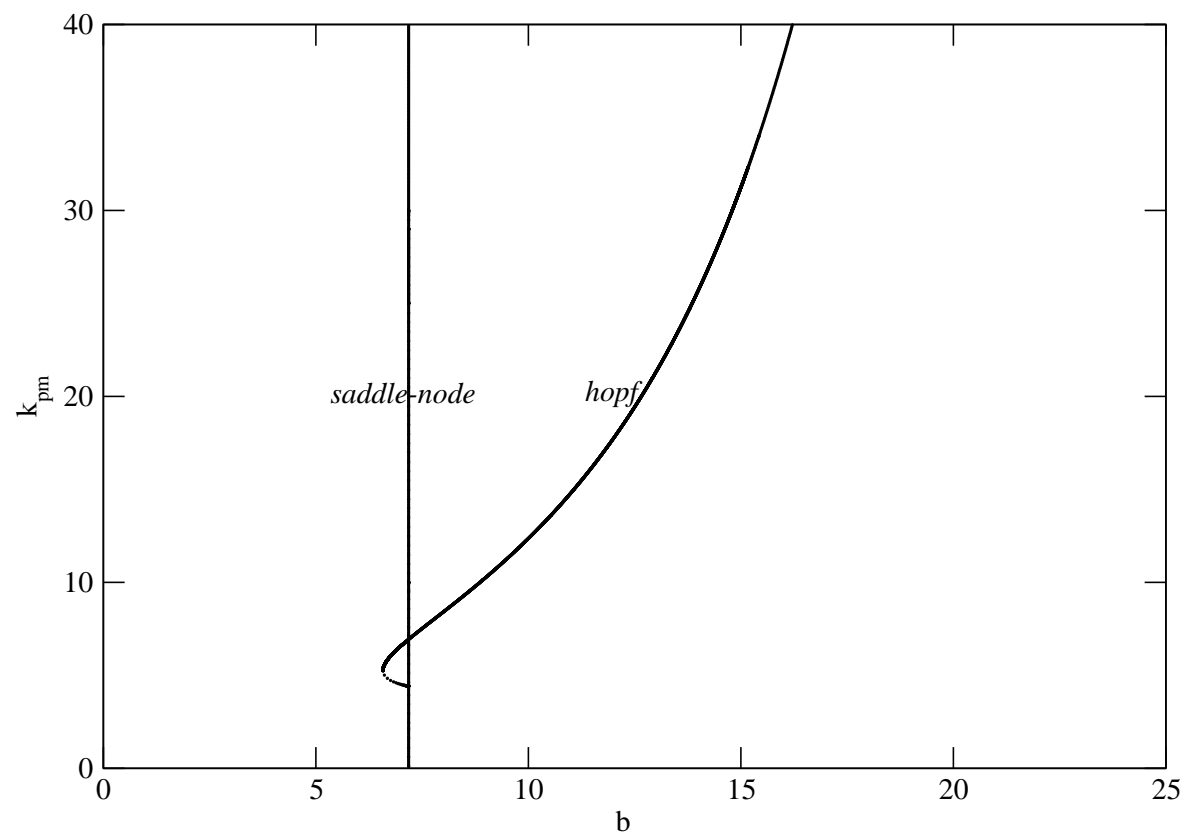

Figure 2.12: A 2 parameter bifurcation plot that varies the strength of early and late inflammatory mediators and follows the saddle-node bifurcation that gives rise to the persistent inflammation fixed point. Only the strength of the late mediators (same $b$, as long as $\theta$ and $w$ remain the same) affects the saddle-node bifurcation. The Hopf bifurcation is affected by both parameters. The Hopf for parameter values $k_{l m}<\approx 7$ is super-critical. 

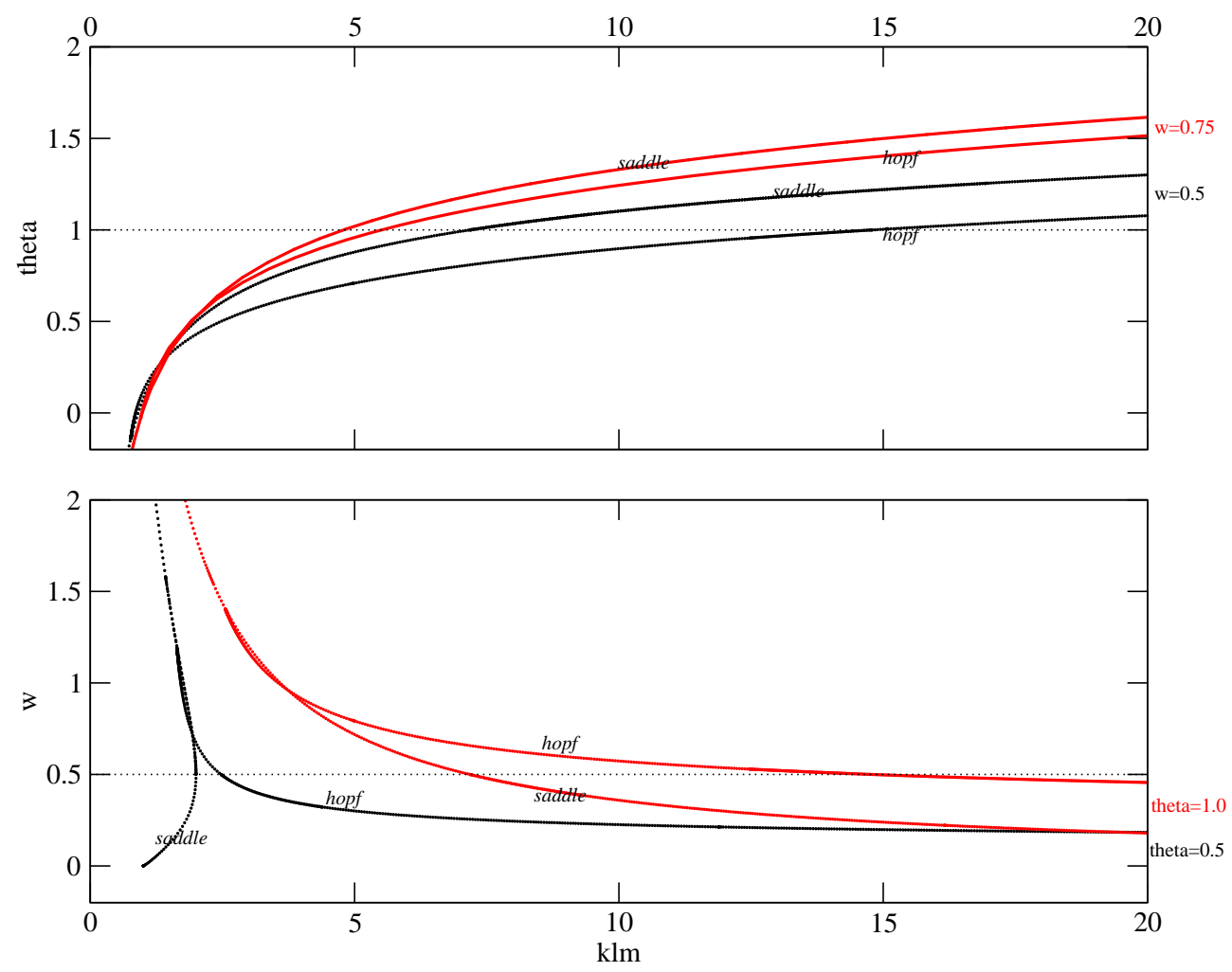

Figure 2.13: Bifurcation plots that vary coupling parameters $\theta$ and $w$ with the strength of late inflammatory mediator $k_{l} m . \theta=1.0$ and $w=0.5$ are used for the simulations. These values result in a larger range of $k_{l m}$ where healthy behavior is supported. 


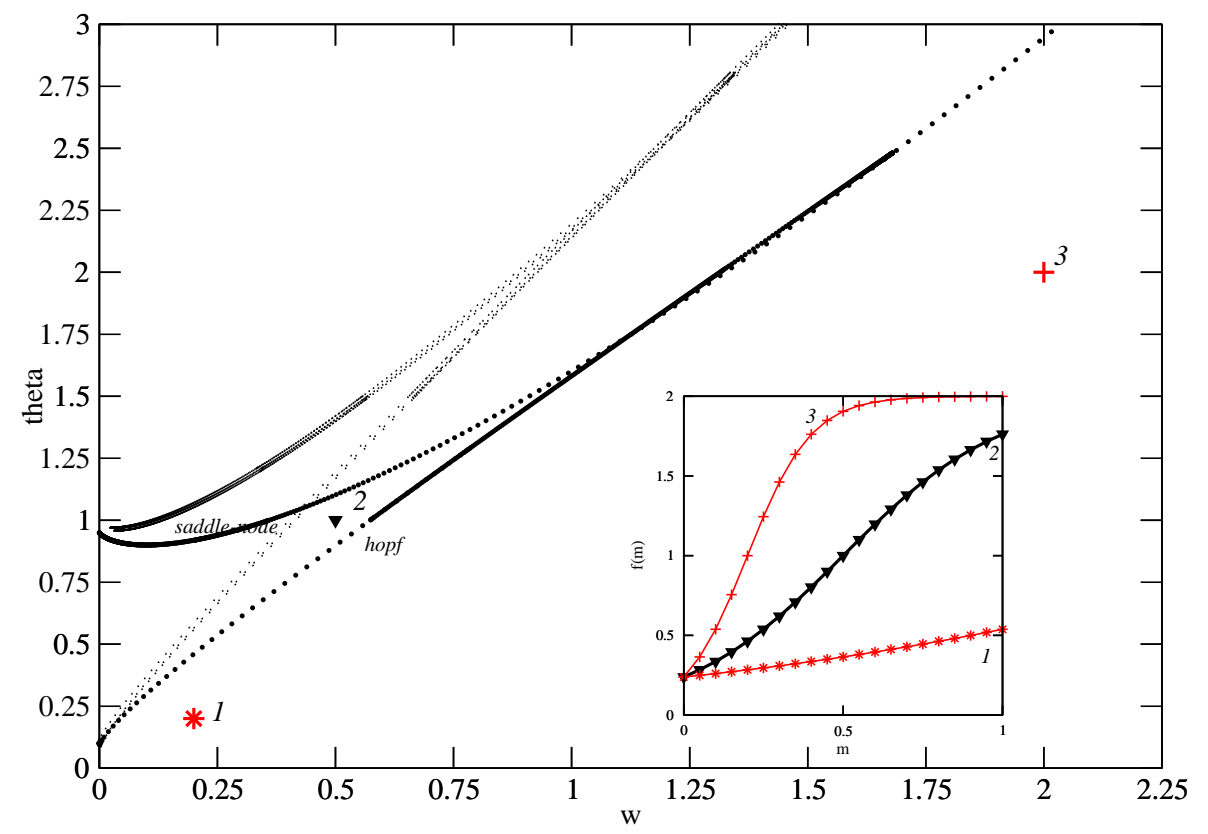

Figure 2.14: A 2 parameter bifurcation plot that varies $\theta$ and $w$ (darker circles : $k_{l m}=10$, lighter circles : $k_{l m}=30$ ). For large $w$ and $\theta$, the two bifurcations coalesce. Representative coupling curves, $f(m)=1+\tanh \frac{(m-\theta)}{w}$ for specific values of $w$ and $\theta$ are shown in the inset (Curve $1 w=0.2, \theta=0.2$; Curve $2 w=0.5, \theta=1.0$; Curve $3 w=2.0, \theta=2.0$ ). $w=0.5$ and $\theta=1.0$ are the values used in the analysis. 
The model has a rich bifurcation structure and exploring it allows us to understand how changing parameters can take the system from one outcome to another. This model shows that in order to have a healthy response to infection the virulence of the pathogen cannot be too strong or too weak, the early pro-inflammatory response cannot be too weak, and the late response cannot be too strong or too weak.

The effect of the strength of early and late pro-inflammatory responses is examined and this model suggests that only the strength of the late pro-inflammatory wave governs predisposition towards a state of persistent inflammation. This is similar to the predictions from the even simpler 2-d model. No matter how exuberant the early wave may be, only controlling the feedback from the late wave can determine whether the outcome is healthy recovery or uncontrolled inflammation. Thus we predict that any therapy for persistent noninfectious inflammation must target the slow pro-inflammatory mediators. In this model, as in the 2-d model, a strong late-inflammatory response ensures elimination of pathogen but introduces the risk of persistent inflammation.

Experiments have found that survival was improved in infected mice when the moderately slow cytokine IL-6 was reduced (Riedemann et al., 2003). However, if too much IL-6 was removed then there was a detrimental effect. This result is consistent with the predictions of the 3-d model in that a small reduction lowered the possibility of a sustained inflammatory response but lowering it too much precluded the possibility of eliminating the bacteria. Evidence also has suggested that down-regulating HMGB-1, a late acting pro-inflammatory mediator might be a potential target for anti-sepsis therapies (Wang et al., 2001). Activated Protein-C which has been recently approved for treatment, is also partially a late antiinflammatory agent (Bernard et al., 2001). Previous therapeutic attempts have mostly focused on down-regulating the early pro-inflammatory mediators and as predicted by the model have not shown great effectiveness (Abraham et al., 1998; McCloskey et al., 1994).

On the other hand, if the patient is suffering from persistent infectious inflammation, then therapies must be aimed at both reducing the pathogen load and the late pro-inflammatory response. In this case, timing of the therapies may be important. It would be necessary to reduce the bacterial load first before reducing the inflammation.

Conversely, low pathogen virulence or a weak immune response can lead to low level 
persistent or recurrent infection. Other theoretical models of infection have similar predictions. Persistence of the tuberculosis bacterium Mycobacterium tuberculosis at low densities for extended periods has been suggested to be the result of slow growth rates (Antia et al., 1996). Similarly, a down-regulated immune response to Helicobacter pylori bacteria has been suggested to result in its persistent colonization of the human stomach (Kirschner \& Blaser, 1995). In the clinical setting, patients in the ICU with decreased host defenses are susceptible to hospital-acquired infections (Schultz et al., 2001). These infections which may be easily resolved in a healthy individual might result in unresolved inflammation and prove fatal to a compromised individual. Immuno-stimulatory therapy might be effective in such a situation.

The model shows that sepsis should be considered as a set of distinct physiological disorders that require separate therapies even though they may have overlapping symptoms. Categorizing septic states based on levels of bio-markers rather than clinical symptoms would be the first step in addressing this problem and effort is ongoing in that direction (Levy et al., 2003; Marshall et al., 2003).

\subsection{A GLOBAL MODEL OF ACUTE INFLAMMATION}

The models discussed in the previous sections are simple and offer general predictions about the design of the inflammatory response. Often, however, results from such models are not specific enough to be of immediate use in understanding complex biological systems. Larger, more complex models incorporating the biological details are being increasingly used to make more specific predictions (Polidori \& Trimmer, 2003; An, 2001). Detailed models are often limited by the amount of accurate biological information and data available to design them. However, they can still be used effectively to gain useful information.

The model discussed in this section is a detailed representation of the processes of acute inflammation. The model reproduces time courses of immune mediators, qualitatively similar to those found in literature. This model can be used to track the time course of specific mediators in response to an insult and specific times and doses of interventions may be suggested to optimize the effectiveness of a given therapy (Kumar et al., 2001; Vodovotz 
et al., 2002). Results from such a model are more valuable to biologists and clinicians than reduced models as the predictions are specific and encompass a variety of mediators.

In this section, the model and its features are discussed and in Chapter 3, its specific applications are elaborated upon.

\subsubsection{Features of the model}

The model consists of 17 equations and captures the behavior of several key mediators using ordinary differential equations. Spatial homogeneity is assumed for simplicity and the mediators are assumed to interact with each other based on the mean field approximation. The underlying assumption is that the time courses of mediators shown by the model represent their concentration in the bloodstream. The effect of systemic inflammation on individual organs or the binding of effectors on cell surfaces by receptors are not modeled and no predictions of such details are made.

The initial instigators of the immune response are the growing pathogen or sterile pathogen products. The model is based on a generic gram negative bacterium that releases endotoxins. An unstable spiraling trajectory formed through Hopf bifurcations (Figs.2.20, 2.21) represents healthy behavior in the global model. Unlike the reduced models, in the simulations of the global model, the oscillating trajectories are clamped using a Heaviside function. The growing pathogen term (Eq.2.39 and Eq.3.3) is multiplied by $H\left(p-p_{0}\right)$ where $p_{0}$ is an arbitrarily set low threshold. This ensures that the pathogen does not re-grow from very low quantities.

The components of the immune system that are modeled are: the first responders which are the phagocytic cells (neutrophils and macrophages), generic early (similar to TNF- $\alpha$ ) and late pro-inflammatory cytokines (similar to IL-6), a generic anti-inflammatory cytokine (similar to TGF- $\beta$ ), oxidizing molecules that can kill pathogen and also cause tissue damage (nitric oxide $\mathrm{NO}$ and oxygen free radical $\mathrm{O}_{2}^{--}$) and components of the coagulation cascade (tissue factor, thrombin and activated protein-C). The model also includes equations for global tissue dysfunction and blood pressure. Tissue dysfunction is caused by the proinflammatory elements in the model and enhances inflammation itself. In general, elevated 
tissue dysfunction and low blood pressure are interpreted as a negative outcome whereas background tissue dysfunction and blood pressure are interpreted as the healthy state. The components of the model with brief descriptions are listed in Appendix 2.7.1.

The interactions modeled are as follows. Resting macrophages $\left(M_{r}\right)$ are activated (activated macrophages are represented by $M_{a}$ ) directly by growing pathogen $(P)$ and sterile pathogen products $\left(P_{e}\right)$. Neutrophils $(N)$ also respond directly to these instigators. These interactions occur at a systemic level where the stimuli enter the circulation and activate circulating monocytes and neutrophils (Parker \& Watkins, 2001). Activated neutrophils reach compromised tissue by migrating along a chemoattractant gradient (Bellingan, 1999). Once activated, macrophages and neutrophils enhance inflammation by producing and secreting effectors that activate these same cells and also other cells, such as endothelial cells. Proinflammatory cytokines - tumor necrosis factor $(T N F)$, interleukin (IL)-6 (IL6), promote immune cell activation and pro-inflammatory cytokine production (Freeman \& Natanson, 2000) These are included as the effects of the TNF and IL6 terms in the model.

The concurrent production of anti-inflammatory cytokines counterbalances the actions of pro-inflammatory cytokines. In an ideal situation, these anti-inflammatory agents serve to restore homeostasis. However, when over produced, they may lead to detrimental immunosuppression (Bone, 1996a; Pinsky, 2001; Volk et al., 2000). The model includes slow-acting anti-inflammatory activity such as active transforming growth factor- $\beta 1$ (TGF- $\beta 1$ ), soluble receptors for pro-inflammatory cytokines, and cortisol (Resting anti-inflammatory molecules represented by $C_{r}$ and activated molecules represented by $C_{a}$ ). It is noted that while activated TGF- $\beta 1$ only has a lifetime of a few minutes, latent TGF- $\beta 1$ is ubiquitous (Roberts \& Sporn, 1996) and can be activated either directly or indirectly by other slower agents such as IL-6 or NO (Luckhart et al., 2003; Villiger et al., 1993; Vodovotz et al., 1999).

Pro-inflammatory cytokines also induce macrophages and neutrophils to produce free radicals. In this model, NO derived from inducible NO synthase, iNOS $\left(N O_{d}\right)$ is directly toxic to bacteria and indirectly to host tissue (Nathan \& Hibbs, 1991; Babior, 2000; Johnson $\&$ Billiar, 1998). The actions of superoxide $\left(\mathrm{O}_{2}^{-}\right)$and other oxidative mechanisms (Babior, 2000) of the pro-inflammatory agents are also accounted for. In the model, the actions of these products can cause direct tissue dysfunction or damage and this induced damage can 
incite more inflammation by activating macrophages and neutrophils (Jaeschke \& Smith, 1997). However, NO can also protect tissue from damage induced by shock (Florquin et al., 1994; Harbrecht et al., 1992; Park et al., 1996), even though overproduction of this free radical causes hypotension (Johnson \& Billiar, 1998).

In endotoxemia, the model assumes that Lipopolysaccharide (LPS) (represented by $P_{e}$ ) enters the bloodstream and incites a system-wide response (Beutler, 2000). LPS is cleared in approximately one hour (Maitra et al., 1981; Ruiter et al., 1981). Circulating neutrophils are activated directly and produce TNF (Djeu et al., 1990). The newly produced TNF combines with LPS to activate macrophages that then secrete pro-inflammatory cytokines including TNF, IL-6. (Cavaillon, 1994). Activated neutrophils, macrophages, and endothelial cells produce NO through iNOS (Bogdan, 2001). The model assumes that locally produced NO is eventually detected as the measured serum end products NO2-/NO3-, and this process depends on the differential induction of eNOS and iNOS in various organs over time (Rees et al., 1995; Santak et al., 1997). In order for TNF to rise and fall within a few hours as it does in Fig. 2.15, the model required an inhibitory agent to suppress TNF production. This was accounted for by saturating mechanisms (Bogdan et al., 1991; de Waal Malefyt et al., 1991) and other slow anti-inflammatory cytokines including IL-6. Previous work has indicated that IL-6 may exert both pro- and anti-inflammatory properties (Opal \& DePalo, 2000). The anti-inflammatory action is assumed to be mediated by inducing or activating TGF- $\beta$ (Villiger et al., 1993) on the surface of neutrophils and macrophages, as has been shown for cytokines such as interferon- $\gamma$ (Chong et al., 1999).

This model is a very incomplete representation of all the processes of inflammation. However, it captures many of the main features of acute inflammation, and can give new insights into the complex interactions of the process. The interactions described in the model are well documented and widely accepted in the biological literature. Their mathematical forms are often based on estimates and documented relative strengths of interactions. There are 100 rate constants or parameters in the model. Of these, 72 determine the strength of interactions. 20 parameters are used to determine strengths and timings of intervention which are not used to run the "base-line" case. 7 determine the thresholds and slopes for hill- type interactions, 1 parameter is used to scale time to correspond to literature values. 
Of the 72 rate constants, some values and ranges of parameters are obtained directly from literature and others are estimated based on relative strengths of interactions and in order to satisfy base-line model behavior. There is uncertainty in the actual values of the parameters that could influence the interpretations from the model. The values of the mediators are not calibrated directly to data and only qualitatively follow expected behavior. Therefore the units in which the mediators are expressed is arbitrary. However, time is marked in hours. The set of mediators chosen to model is also subjective and arguably the role of early anti-inflammatory cytokines has been underestimated. However, the variables chosen also represent the major players which could not have been ignored.

Despite the various limitations explained above, the model is robust to small parameter changes and is a useful systemic description of acute inflammation. Several meaningful predictions, both intuitive and non-intuitive have been obtained from this model, some of which are discussed below. This model can be used to test hypotheses about the interactions and test the systemic effect of modulating specific mediators.

\subsubsection{Simulations}

The features for which this model was trained are summarized below. The healthy state, persistent non-infectious inflammation and persistent infectious inflammation were required to arise on increasing pathogen growth rate. Persistent inflammation was characterized by elevated levels of immune mediators, tissue dysfunction and low blood pressure. The time courses of early and late pro-inflammatory cytokines and nitric oxide were modeled according to time courses obtained in the general biological literature. The response of the immune system to sterile pathogen toxins was also used to determine some rate constants.

In the case of a moderate pathogen, healthy behavior is expected. In this model a heaviside function is used to cut off pathogen growth when it falls below a threshold. On increasing pathogen growth rate, the immune mediators stay elevated and persistent noninfectious inflammation is observed. The concentrations of pathogen, early phagocytic cells, cytokines, nitric oxide and tissue dysfunction in both these scenarios is shown in Figure 2.15 and Figure 2.16. 


\subsubsection{Connections}

The reduced models and the detailed models exhibit qualitatively similar behavior in terms of the scenarios they are able to reproduce and the parameter changes that take the system from one scenario to the other. Several features are conserved between the models and later in this section, some simulations and bifurcation plots from the detailed models are compared to those from the 3-d model.

The interactions of the 3 - $\mathrm{d}$ model are obtained from reducing the global detailed model. In the global model, an invading pathogen (initial condition of the variable $P$ ) triggers an immune response by activating the resting phagocytic cells (resting macrophages $M_{r}$ ) get converted to activated forms. (activated macrophages $M_{a}$ ). These cells directly and through cytotoxic products $\left(\mathrm{NO}\right.$ and $\mathrm{O}_{2}$ ) clear the pathogen. This results in the production of toxic pathogen products (endotoxin $P_{e}$ ) which further trigger immune response. This initial loop is simplified in the 3 - $\mathrm{d}$ model by assuming a growing pathogen $p$ triggering a reaction from a single immune mediator $m$ which can directly clear pathogen.

In the global model, the early immune responses trigger further inflammatory products such as the cytokines TNF- $\alpha$ and IL-6 and compensatory anti-inflammatory cytokines TGF$\beta$. Further downstream, the coagulation cascade (tissue factor $T_{f}$, thrombin $T_{h}$ and activated protein-C $\left.P_{c}\right)$. These mechanisms along with increasing tissue dysfunction $(D)$ and decreasing blood pressure $(B)$ further enhance inflammation. For instance, macrophages increase rate of production TNF- $\alpha$ (at rate $k_{t n f m a}$ ) which in turn increase tissue factor $\left(k_{t f t n f}\right)$ which increases thrombin $\left(k_{t f t h}\right)$ and that increases IL-6 $\left(k_{6 t h}\right)$ which finally increases the activation rate of macrophages $\left(k_{m 6}\right)$. Such loops are simplified in the 3-d model through a single late inflammatory mediator, $l$ that enhances the early mediator $m$. The saturating effects of anti-inflammatory agents such as TGF- $\beta$ and activated protein-C are simulated through saturating functions in the equations of both $m$ and $l$.

All the scenarios that are possible in the 3-d model can be generated in the global model. The variables in the larger model include more than one primary mediator (macrophages and neutrophils), several secondary mediators and then another wave of pro- and anti inflammatory mediators. So, scenarios not seen in the reduced model such as fixed points somewhere 
between clearly immuno-suppressed and clearly persistent infectious inflammation are also possible in the global model. But going from one scenario to the other follows more or less the same pattern in both models. The scenarios of persistent inflammation (infectious and non-infectious), recurrent infection, immune-suppression as seen in the global model are shown in Figs. 2.15 - 2.19. The structure of the phase space of the global model is clearly complicated and is beyond the scope of detailed analysis. The persistent inflammation outcomes are obtained by decreasing pathogen susceptibility (decreasing parameters $k_{p m}, k_{p n o}$ and $\left.k_{p 02}\right)$ and the immuno-deficient outcomes are obtained by decreasing early immune response to pathogen and pathogen products (decreasing parameters $k_{m p}, k_{n p}$ and $k_{t f p e}$ ). The bifurcation diagrams of the global model reveal the structure of fixed points which is very similar or in some cases identical to that of the 3-d model (Figs. 2.21, 2.20).

The strength of the late inflammatory response in the 3 - $\mathrm{d}$ model is crucial in determining the predisposition of a system to persistent inflammation. More than one variable could qualify as the strength of "late response" in the detailed model. Several parameters such as $k_{6 m}$ which governs the stimulation of IL-6 by macrophages or $k_{d c p}$ which governs the damage caused by TNF- $\alpha$ can be changed and these result in qualitatively similar behavior as seen in the reduced models: stable oscillations representing recurrent infection are followed by unstable oscillations representing healthy behavior followed by the creation of a persistent inflammation fixed point. In particular, the bifurcation plot of the parameter $k_{d t h}$, which governs the strength at which thrombin stimulates damage, is shown in Figure 2.21. It can be seen that it is very similar to Figure 2.10 which shows the variation of the strength of the late inflammatory mediator in the reduced model.

The global model also shows similar dynamics when the early immune response is changed. The parameter $k_{m p}$ which governs the pathogen stimulation of macrophages is varied as shown in Figure 2.20. Low stimulation causes recurrent infection and higher stimulation causes healthy behavior. However just as it is observed in the reduced model (Figure $2.9)$, even on increasing $k_{m p}$ to very large values, the possibility of a healthy outcome is never lost.

Detailed results based on both the global and reduced models have been discussed. It is expected that the validity of the above conclusions not change with small changes in the 
functional form of the model. This concept is formally referred to as "structural stability". The general idea is that similar dynamics (topological equivalence) is shown by a system even when the functional forms (or parameters) of the system are slightly varied (Kuznetsov, 1998). Necessary and sufficient conditions for structural stability in 2 dimensions (Peixoto, 1962; Arrowsmith \& Place, 1990) can be stated as follows. 1) All fixed points are hyperbolic 2) All closed orbits are hyperbolic 3) There are no orbits connecting saddles. While the local conditions (1) and (2) are satisfied in the 2-d model (2.4-2.3), the global condition (3) is not satisfied. So though local structural stability can be proved in compact sets in the 2-d model, it is not globally structurally stable.

In dimensions higher than 2 only "sufficient" conditions for structural stability can be stated (Garcia \& Teixeira, 2004). These are the Morse-Smale conditions and the structural stability of the system away from local bifurcations (such as the Hopf and saddle-node bifurcations seen in the 3-d (2.8-2.10) and global models (2.40)) can also be proved (Afrajmovich et al., 1988). These conditions are stated as follows. 1) There is only a finite number of fixed points and periodic trajectories 2) All such fixed points and periodic trajectories are hyperbolic 3) the stable and unstable manifolds intersect transversally. While conditions (1) and (2) are satisfied in the models (2.8-2.10) and (2.40), condition (3) can be proved formally only on further analysis of the stable and unstable manifolds (Day, 2004).

It is noted however, that the specific nature of the interactions in the 3 -d model are not essential for the qualitative dynamics found. There is some flexibility in the functional form chosen. As an example, the factor $1-m$ in (2.9) may be replaced by $1 /(1+m)$ or the form of the coupling function (2.11) can be changed without affecting the conclusions. In this study, we have shown qualitatively similar dynamics in the global and reduced models only through simulations and similarities in bifurcation diagrams.

\subsection{LIMITATIONS OF THE MODELS}

The modeling approach has proven very useful in understanding the dynamics of acute inflammation. However, there are certain limitations as discussed below.

In the models above, trajectories that were unstable spirals were interpreted as healthy 

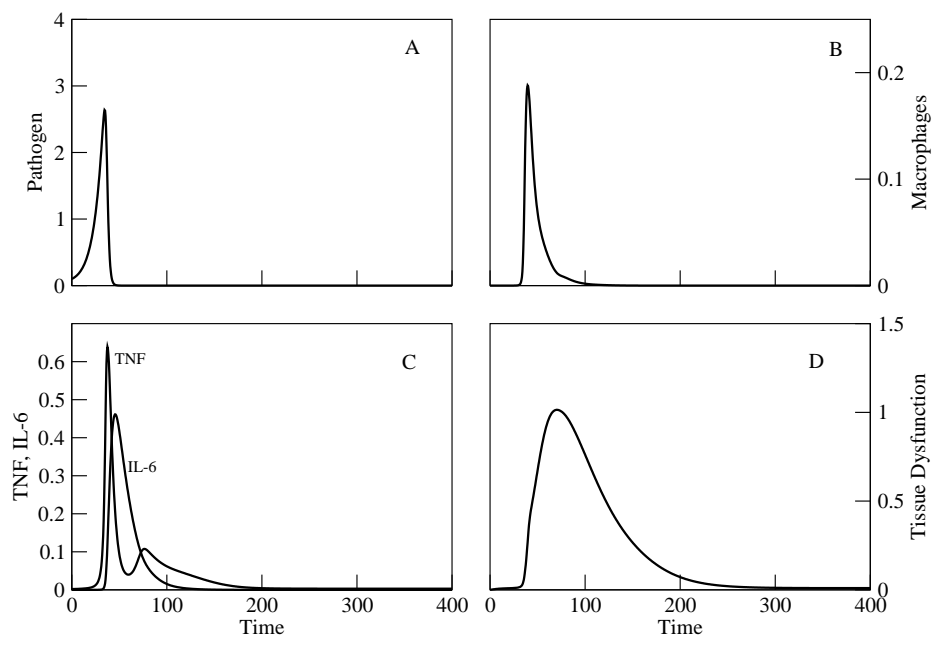

Figure 2.15: Simulation of a healthy inflammatory response to Gram-negative bacterial infection: The system is infected by a low dose of Gram-negative bacteria (e.g. E. coli, Panel A), which leads to activation of macrophages (Panel B). The macrophages elaborate cytokines (Panel C), including rapidly produced pro-inflammatory cytokines (CP, e.g. TNF- $\alpha$ ), more slowly produced pro-inflammatory cytokines (CPL, e.g. IL-6), and anti-inflammatory cytokines (Ca, e.g. TGF- $\beta 1$ ). Tissue damage/dysfunction (Panel D) rises due to the actions of pro-inflammatory cytokines and effectors, but then decreases due to the actions of antiinflammatory cytokines. Thus, homeostasis is restored after pathogens are cleared. 

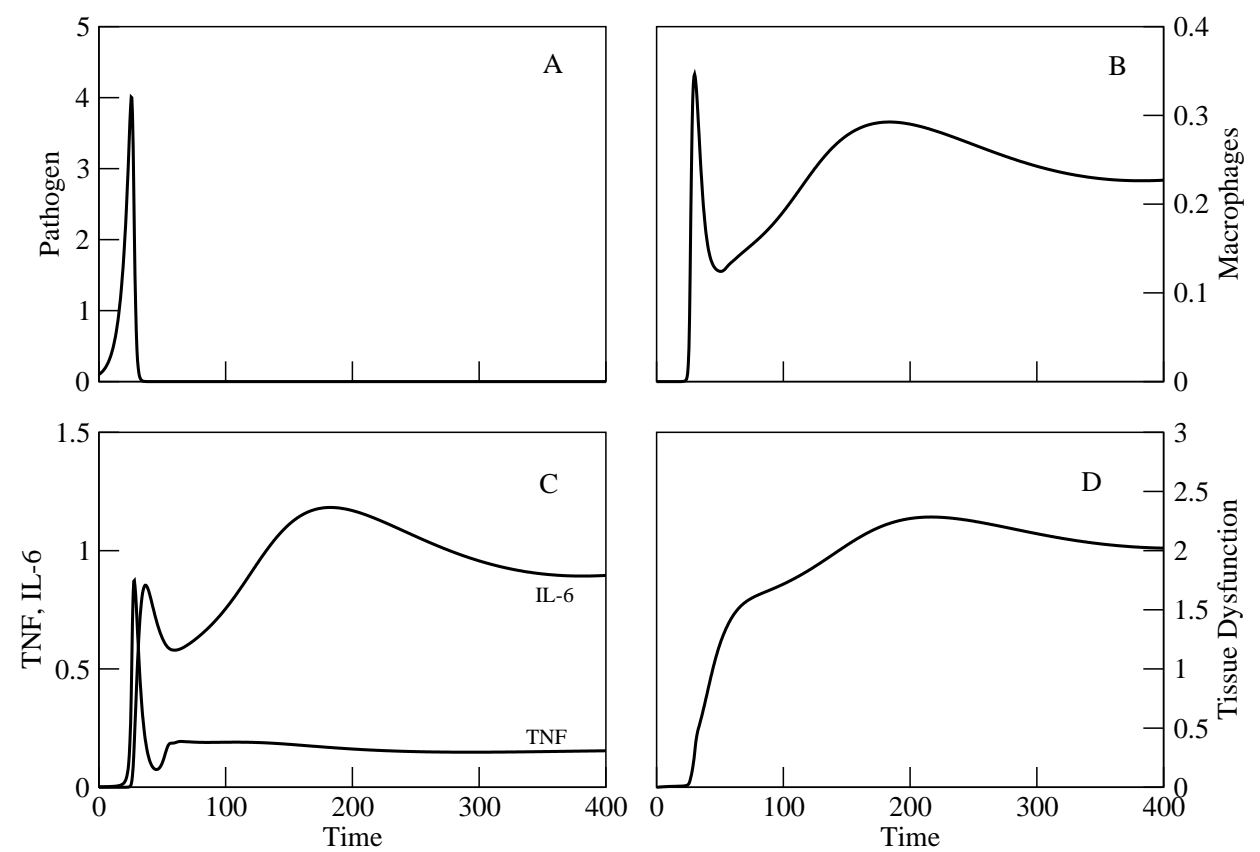

Figure 2.16: Simulation of persistent non-infectious inflammation: The system is infected by a high dose of Gram-negative bacteria (e.g. E. coli, Panel A), which leads to activation of macrophages (Panel B). The macrophages elaborate cytokines (Panel C), including rapidly produced pro-inflammatory cytokines $(\mathrm{CP}$, e.g. $\mathrm{TNF} \alpha)$, more slowly produced proinflammatory cytokines (CPL, e.g. IL-6), and anti-inflammatory cytokines (Ca, e.g. TGF$\beta 1$ ). Tissue damage/dysfunction (Panel D) rises due to the actions of pro-inflammatory cytokines and effectors, and unlike the case in Fig. 2.15 is not reduced. Thus, the inflammatory response is sustained, with ensuing adverse outcome. 

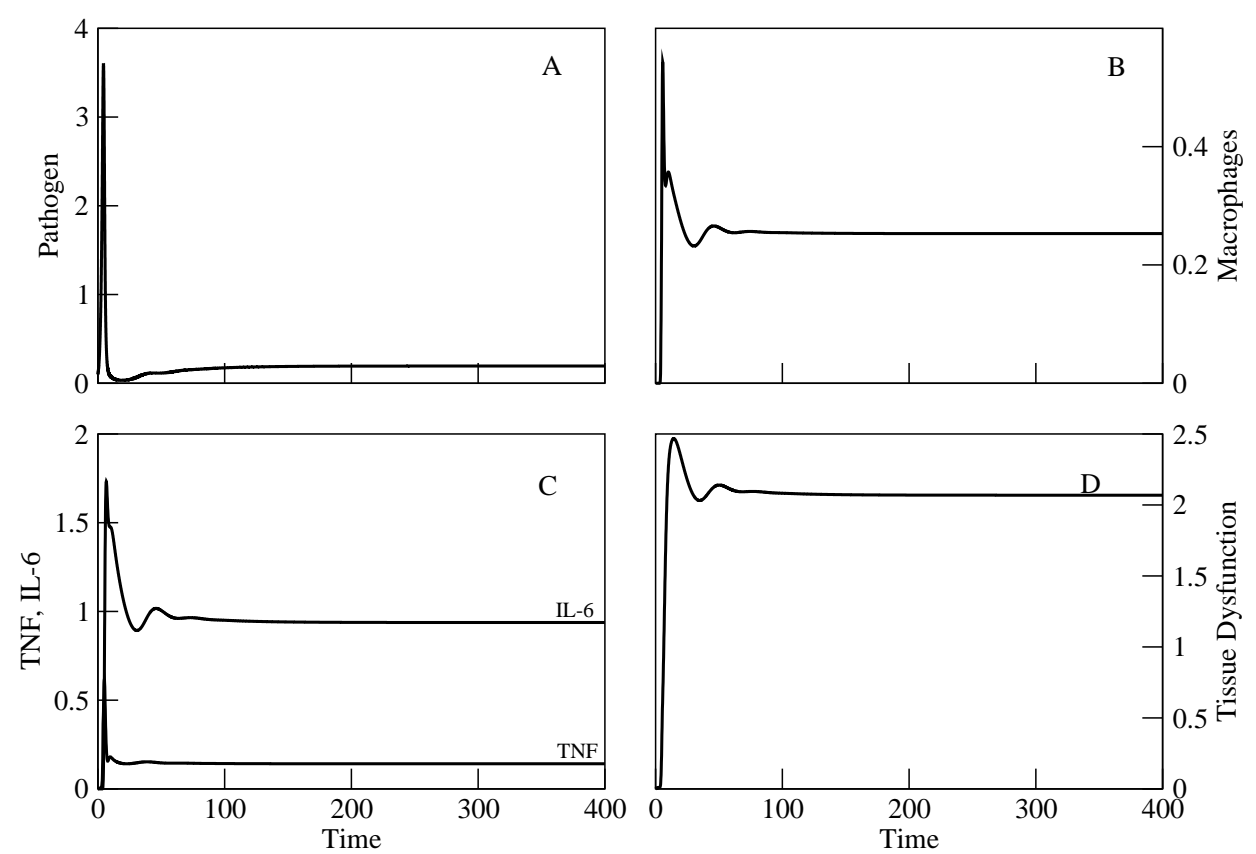

Figure 2.17: Simulation of persistent infectious inflammation: The system is infected by a very fast growing Gram-negative bacteria (e.g. E. coli, Panel A), Macrophages are shown in Panel B, Cytokines in Panel C and Tissue damage/dysfunction in Panel D. Unlike the case in Fig. 2.15 inflammatory mediators are not reduced as the response elaborates. Also, unlike Fig. 2.16, the pathogen load also does not reduce and active infection persists despite severe inflammation. The inflammatory response and infection are sustained, with ensuing adverse outcome. 

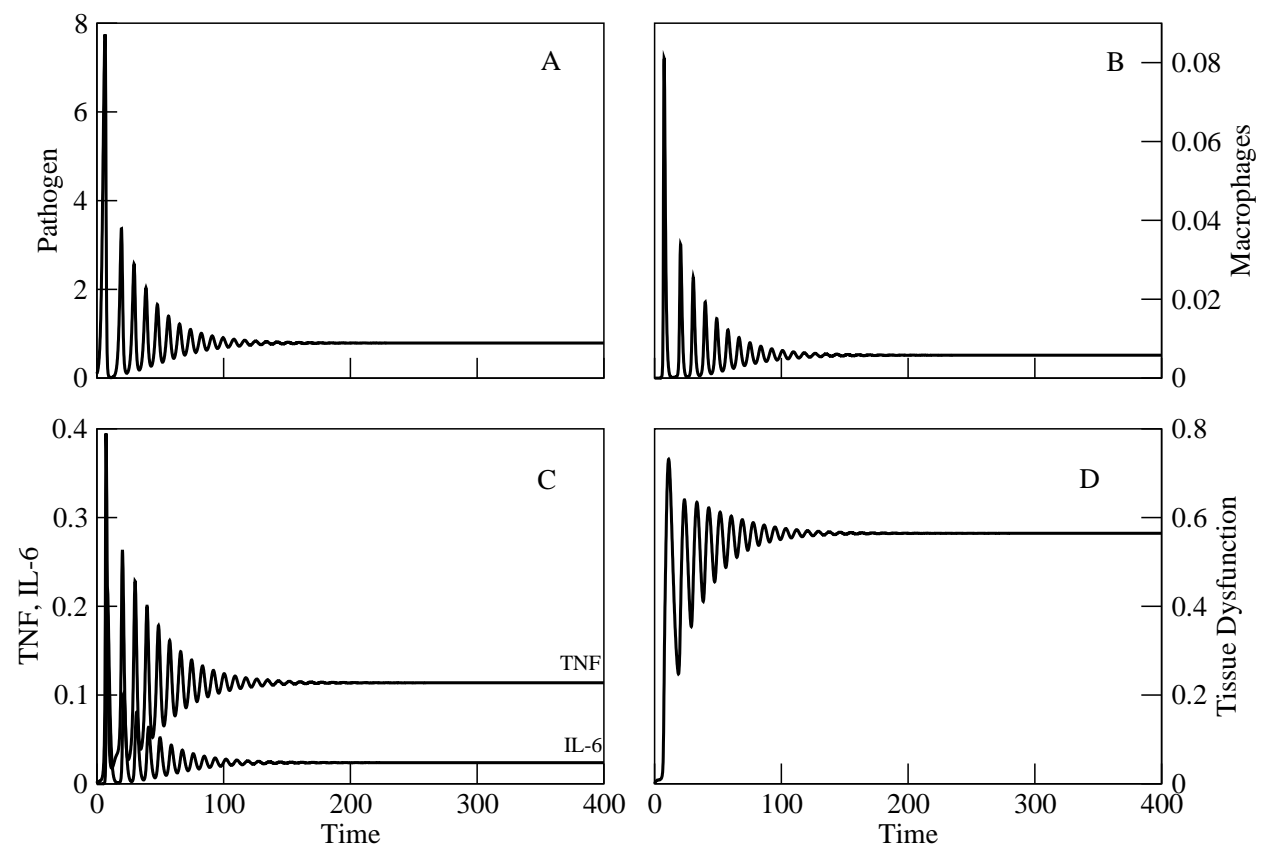

Figure 2.18: Simulation of recurrent infection: A virtual patient is infected by a slow growth rate Gram-negative bacteria, (Panel B). Macrophages are shown in Panel B, Cytokines in Panel C and Tissue damage/dysfunction in Panel D. The low growth rate infection does not elicit a strong response and persistent low-grade inflammation exists. 

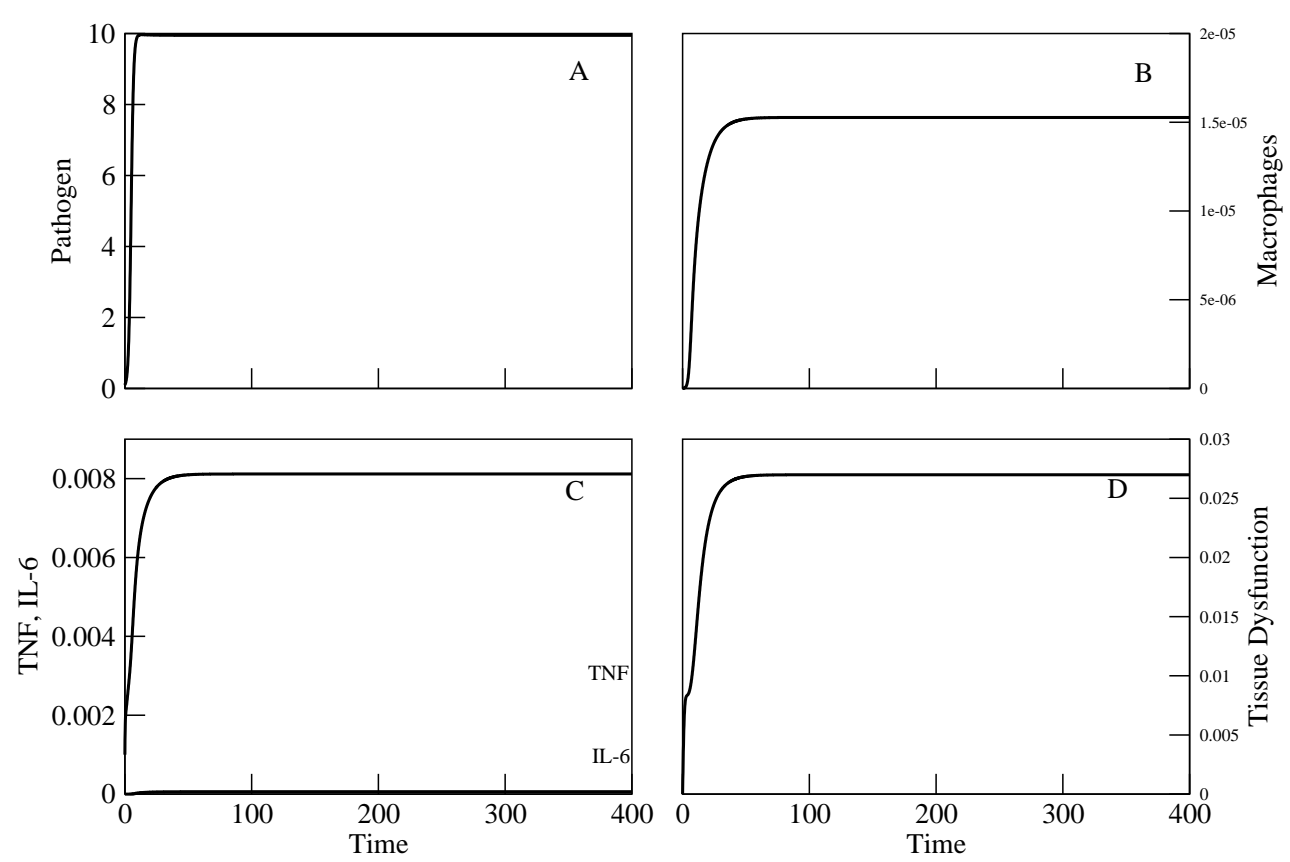

Figure 2.19: Simulation of severe immuno-deficiency: The system is infected by a moderate dose of Gram-negative bacteria (Panel A). The immune response is very weak (Panels B,C and D) and this results in unchecked pathogen growth and background levels of inflammatory mediators. An adverse outcome is expected to ensue in the organism due to large amounts of pathogen, even though the damage term of the model in Panel D remains low. 


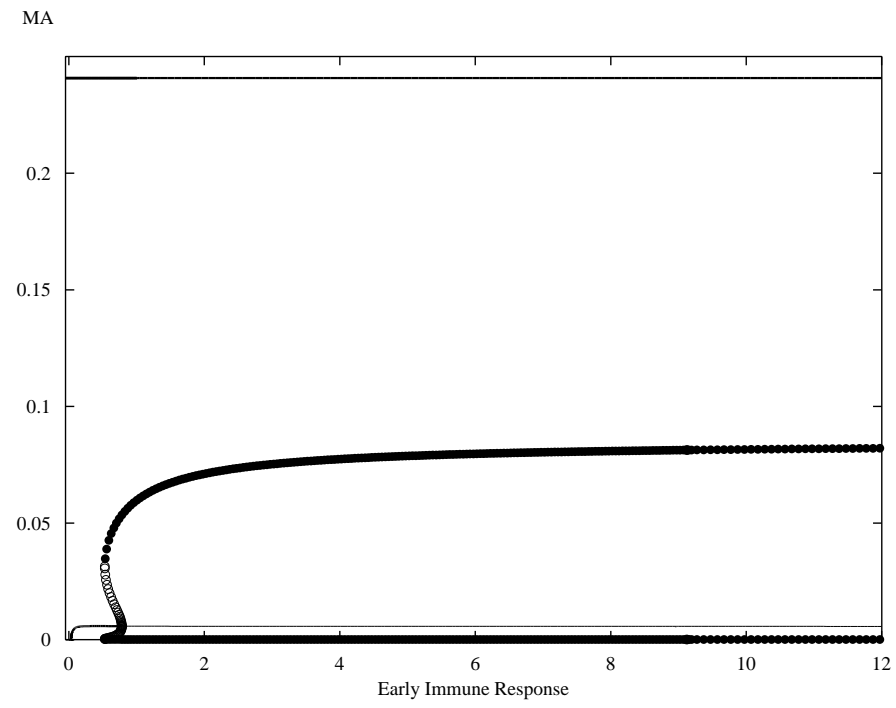

Figure 2.20: The bifurcation plot varying the strength of macrophage and neutrophil response to pathogen and tissue-factor response to endotoxin. Even on increasing the response to very high values, healthy oscillations are always a possible outcome.

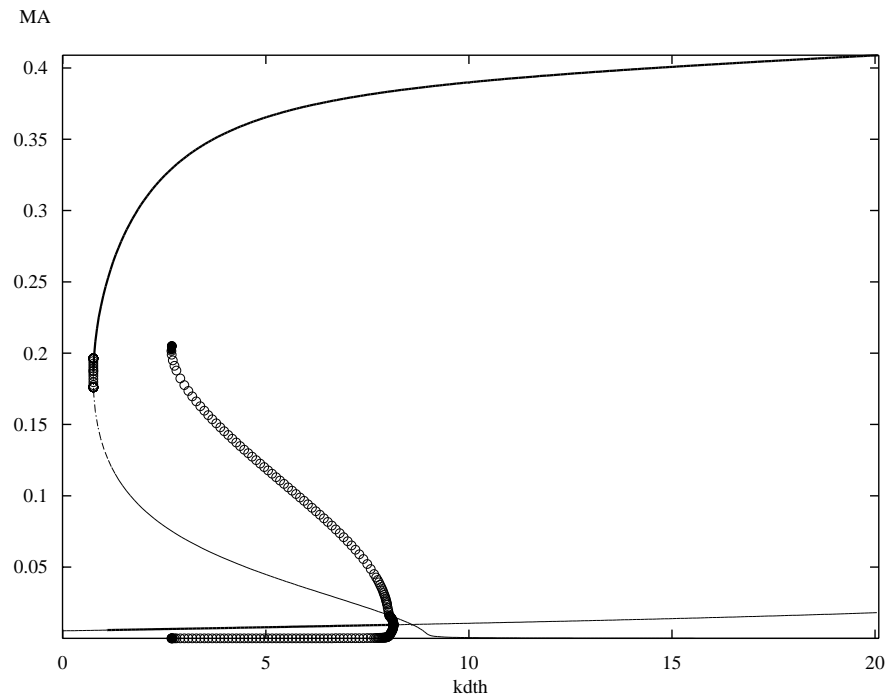

Figure 2.21: The bifurcation plot varying the strength of thrombin stimulation of damage is very similar to that of varying the strength of the late inflammatory response in the reduced model. 
outcomes. In general, these trajectories do take the pathogen values very close to 0 very soon, and can be interpreted as healthy outcomes. However, the trajectories of some initial conditions might wind longer around the fixed point before reaching $p=0$. Such trajectories cannot be immediately interpreted as healthy behavior and might be considered closer to recurrent infection behavior.

Another draw-back is that though the outcomes of the reduced and global models have been correlated to clinical conditions, strict correspondence is difficult to establish. The persistent inflammation fixed point is interpreted as being in the "shock" state and leading to death, though the exact pathway to death is not clearly modeled.

Some important aspects of the immune response have also been ignored in the models above. For instance, the role of anti-inflammatory mediators could be more clearly examined by explicitly including such a variable in a reduced model. Elements of adaptive immunity may also be added to the global model to understand its systemic effects. Effort is ongoing in that direction (Day, 2004).

The effects of fluctuations at low numbers have been ignored by the above models and arbitrary thresholds have been used to ensure elimination of pathogen. Chapter 4 discusses a more fundamental approach to deal with this problem. 


\subsection{APPENDIX TO THE 2-D MODEL}

The most general 2-dimensional model with positive coefficients is described and the stability properties of its fixed points are analyzed.

$$
\begin{aligned}
\frac{d p}{d t} & =k_{p} p-k_{p m} m p-k_{s} p^{2} \\
\frac{d m}{d t} & =k_{m p} m p-k_{m} m+k_{f} m^{2} .
\end{aligned}
$$

The nullclines of the most general model are

$$
\begin{aligned}
0 & =p\left[p-\frac{k_{p}-k_{p m} m}{k_{s}}\right] \\
0 & =m\left[p-\frac{k_{m}-k_{f} m}{k_{m p}}\right]
\end{aligned}
$$

and the fixed points (FP) are:

FP 1: $p=0, m=0$ (exists always)

FP 2: $p=\left(k_{m}-k_{f} m^{*}\right) / k_{p m}, m^{*}=\left(k_{m} k_{s}-k_{m p} k_{p}\right) /\left(k_{f} k_{s}-k_{p m} k_{m p}\right)$ (exists always; when $k_{f}=0$ is modified to $p=k_{m} / k_{m p}, m=\left(k_{p} k_{m p}-k_{m} k_{s}\right) /\left(k_{p m} k_{p m}\right)$; when $k_{s}=0$ is modified to $p=\left(k_{m}-\frac{k_{f} k_{p}}{k_{p m}}\right) / k_{m p}, m=k_{p} / k_{p m}$; and is $p=k_{m} / k_{m p}, m=k_{p} / k_{p m}$ when both $k_{f}$ and $k_{s}$ are 0$)$

FP 3: $p=0, m=k_{m} / k_{f}\left(\right.$ does not exist if $\left.k_{f}=0\right)$

FP 4: $p=k_{p} / k_{s}, m=0$ (does not exist if $\left.k_{s}=0\right)$.

The Jacobian of $(2.18,2.19)$ can be written out to determine the stability of the fixed points listed above.

$$
\left(\begin{array}{cc}
k_{p}-k_{p m} m-2 k_{s} p & -k_{p m} p \\
k_{m p} m & k_{m p} p+2 k_{f} m-k_{m}
\end{array}\right)
$$

Plugging $p=0, m=0$ in the Jacobian, the eigen-values of FP 1 can be obtained as $k_{p},-k_{m}$ which is always a saddle. FP 3 has eigen-values $k_{p}-\left(\left(k_{p m} k_{m}\right) / k_{f}\right), k_{m}$. So FP 3 is a saddle if $k_{f}<\left(k_{p m} k_{m}\right) / k_{p}$ and unstable otherwise. FP 4 has eigen-values $-2 k_{p},\left(\left(k_{m} p k_{p}\right) / k_{s}\right)-k_{m}$. So FP 4 is stable if $k m p / k_{m}<k_{s} / k_{p}$ and a saddle otherwise. 
For FP 2, the case where $k_{s}=0$ is examined first for simplicity and then generalized to when $k_{s} \neq 0$. When $k_{s}=0$, in order that FP 3 lie in the positive quadrant, $k_{f}<k_{p m} k_{m} / k_{p}$. FP 3 can be plugged into the Jacobian (2.22) and a characteristic equation for its eigenvalues can be obtained as follows: $\lambda^{2}-\left(k_{f} k_{p} / k_{p m}\right) \lambda+\left(k_{p} k_{m}-\left(k_{p}^{2} k_{f}\right) / k_{p m}\right)=0$. To ensure an unstable spiral, the real part of $\lambda<0$ or $k_{f}<0$; to ensure that FP 2 is a spiral, $\lambda$ should be a complex number. The condition $k_{f}<k_{p m} k_{m} / k_{p}$ is a stronger one and we retain that for the behavior of interest i.e., unstable spiral in the positive quadrant. When there is a positive saturation term for pathogen growth, $k_{s}>0$, the additional conditions $k_{f}>k_{s}$ is specified for the negative spiral to exist.

In the case where the term $\theta p^{2}$ is added to the $p$ equation rather than subtracting the logistic term $k_{s} p^{2}$, FP 2 is an unstable spiral unless $k_{f}<\theta$ i.e. $k_{f}$ is a smaller negative number than $\theta$.

$\left(k_{f}, k_{s}\left(k_{s}\right.\right.$ being positive or negative) determine the slopes of the nullclines that intersect to form FP 2. The stability of FP 2 depends on the angle at which the nullclines intersect and hence on $k_{f}$ and $k_{s}$. FP 2 is an unstable spiral as long as $k_{f}>k_{s}$ and stable when $k_{f}<k_{s}$ and exhibits centers when $k_{f}=k_{s}$ ).

\subsection{APPENDIX TO THE 3-D MODEL}

In this section, a detailed phase-plane analysis of the 3 dimensional model of acute inflammation is carried out.

\subsubsection{Eigenvalues of Fixed Points}

The Jacobian matrix of the model (2.8) - (2.10) is

$$
\left(\begin{array}{ccc}
k_{p}(1-2 p)-k_{p m} m & -k_{p m} p & 0 \\
k_{m p} m(1-m) & \left(k_{m p} p+l\right)(1-2 m)-1 & m(1-m) \\
0 & \frac{k_{l m}}{w} \operatorname{sech}^{2}\left(\frac{m-\theta}{w}\right) & -k_{l}
\end{array}\right)
$$


2.6.1.1 $\quad$ FP $1 \quad$ For FP1: $p=0, m=0, l=\frac{k_{l m}}{k_{l}}(1+\tanh (\theta / w))\left(\equiv l^{0}\right)$, the Jacobian is

$$
\left(\begin{array}{ccc}
k_{p} & 0 & 0 \\
0 & l^{0}-1 & 0 \\
0 & \frac{k_{l m}}{w} \operatorname{sech}^{2} \frac{\theta}{w} & -k_{l}
\end{array}\right)
$$

This matrix is lower-triangular and the eigenvalues are $k_{p}, l^{0}-1,-k_{l}$. The fixed point is never stable as one of the eigenvalues $k_{p}$ is always positive.

2.6.1.2 FP 2 For FP2: $p=1, m=0, l=l^{0}$, the Jacobian is

$$
\left(\begin{array}{ccc}
-k_{p} & -k_{p m} & 0 \\
0 & \left(k_{m p}+l^{0}\right)-1 & 0 \\
0 & \frac{k_{l m}}{w} \operatorname{sech}^{2} \frac{\theta}{w} & -k_{l}
\end{array}\right)
$$

The characteristic polynomial for the eigenvalue $\lambda$ is

$$
\left(-k_{p}-\lambda\right)\left(-k_{l}-\lambda\right)\left(k_{m p}+l^{0}-1-\lambda\right)=0
$$

with roots: $\lambda=-k_{p}, \lambda=-k_{l}$ and $\lambda=k_{m p}+l^{0}-1$. Thus the condition for stability of FP2 is $k_{m p}<1-l^{0}$ or $k_{m p}<1-\frac{k_{l m}}{k_{l}}(1-\tanh (\theta / w))$. The other two eigenvalues are always negative. 
2.6.1.3 FP 3 FP3 is the solution of the following equations,

$$
\begin{aligned}
p & =1-a m \\
m & =1-\frac{1}{k_{m p} p+l} \\
l & =b f(m)
\end{aligned}
$$

This may be reduced to a single transcendental equation for $m$ :

$$
m=1-\left(\frac{1}{k_{m p}}\left(\frac{1}{1-a m}\right)+b f(m)\right) .
$$

All the parameter combinations affect the position and eigenvalues of FP 3. Parameter choices for which $p$ or $m$ of FP 3 is negative are unphysical. FP 3 could also have real or complex eigenvalues. FP 3 has complex eigenvalues and can support oscillatory behavior when (2.16) intersects (2.17) such that $m$ of FP 3 is less than $m$ of FP 4 (Fig. 2.7). FP 3 can undergo Hopf bifurcations in this part of the phase-space - the subcritical Hopf bifurcations on varying the three parameter combinations are shown in Figs. 2.8, 2.9, and 2.10.

A supercritical Hopf bifurcation occurs when we change the coupling curve (2.11) so that $\theta$ is small (low threshold for activation) and $w$ is large (shallow coupling curve). In this case, for increasing $b$ while keeping $a$ and $k_{m p}$ fixed at moderate values, a state of persistent non-infectious inflammation will follow recurrent infection and a small zone of stable limit cycles. (Varying the other parameters, for small $\theta$ and large $w$, does not result in Hopf bifurcations). In the case of small $w$ (sharp coupling curve) and small $\theta$ (low threshold of activation), it is possible that more than one fixed point satisfies the conditions for FP 3 . In this case, curve (2.17) intersects line (2.16) twice before dipping below zero. On varying the parameter combinations, recurrent infection is bistable with persistent inflammation and healthy behavior is never supported. These cases are discussed in greater detail in Appendix 2.6.2.1. In our numerical examples, we have considered moderate $w$ and large $\theta$, which result in subcritical Hopf bifurcations. 
2.6.1.4 FP 4 and FP 5 These fixed points are the solutions of the following equations,

$$
\begin{aligned}
p & =0 \\
m & =1-\left(\frac{1}{k_{m p} k_{p m} p+l}\right) \\
l & =\frac{k_{l m}}{k_{l}} f(m)
\end{aligned}
$$

This may be reduced to one transcendental equation

$$
b f(m)=\frac{1}{1-m}
$$

Equation (2.33) may have 0, 1 or 2 solutions depending on the parameters. Approximate solutions can be found for (2.33) by substituting a piece-wise linear function such as $f(m)=$ $0, m<\theta-w ; f(m)=1+\frac{(m-\theta)}{w}, \theta-w<m<\theta+w ; f(m)=2, m>\theta+w$. Using the above approximate function and assuming that $\mathrm{FP} 4$ and 5 occur in region $\theta-w<m<\theta+w$, we arrive at the following expressions for FP 4 and 5.

$$
\begin{aligned}
p & =0 \\
m & =\frac{q \mp \sqrt{q^{2}-\frac{4 w}{b}}}{2} \\
l & =b f(m)
\end{aligned}
$$

where $q \equiv 1-\theta+w$.

This gives $b>4 w / q^{2}$ as a lower limit on $b$ for the existence of FP 4 and 5 . When there are two solutions, they are formed through a saddle-node bifurcation as shown in Fig. 2.10. FP 4 is a saddle point and FP 5 is a stable node.

The eigenvalues of FP 4 and 5 can be found by substituting the solutions $m=m^{0}$ of (2.33) into the Jacobian (2.23). One of the eigenvalues is $\lambda_{1}=k_{p}-k_{p m} m^{0}$ and the other two are functions of the parameters $k_{l}, k_{l m}, \theta$ and $w$. We note that $k_{m p}$ does not appear in the expressions for the position or eigenvalues of FP 4 and 5. 


\subsubsection{Regimes for healthy response}

Trajectories can be interpreted as healthy when FP 3 supports unstable oscillations as an unstable spiral or as a stable spiral surrounded by an unstable limit cycle. The unstable limit cycle is lost when it undergoes a non-generic homoclinic bifurcation when it collides with FP 1. The regimes of the various parameters (for fixed $\theta$ and $w$ ) which maintain healthy behavior are discussed below.

For healthy behavior, a should be large enough so that FP 3 is to the left of FP 5 in Fig. 2.7 or $(1 / a)<\left(q-\sqrt{q^{2}-4 w / b}\right) / 2$. Increasing $k_{m p}$ even over a large range, does not make FP 3 unphysical, nor does FP 3 lose its complex eigenvalues - thus even for very large $k_{m p}$, healthy oscillations are supported. The upper limit for $a$ and the lower limit for $k_{m p}$ to maintain healthy oscillations are difficult to find in closed form as these variations result in the radius of the limit cycle increasing and oscillations being lost through a non-generic homoclinic bifurcation.

The strength of the late immune response $b$ should not be too high or too low. If $b$ is too high, then FP 3 has $p<0$ and hence healthy behavior cannot be supported. In order for the late immune response to not be too high, we require that $p$ of condition (2.17) be positive so that FP 3 is not unphysical. Applying this to (2.17) gives the condition

$$
b<\frac{1}{1-\tanh (\theta / w)}
$$

If $b$ is too low, FP 4 and 5 are not yet formed and (2.17) is well above $p=0$ so FP 3 remains the global attractor and is a stable spiral. To avoid too low of a response, we would like FP 4 and 5 to exist so that unstable oscillations from FP 3 are eventually attracted to FP 5 . Thus the condition for existence for FP 4 and 5, as calculated above in 2.6.1.4, is

$$
b>\frac{4 w}{(1-\theta+w)^{2}}
$$


2.6.2.1 Effect of varying the coupling curve The shape of the coupling curve (2.11) (i.e. parameters $\theta$ and $w$ ) can alter the results. We used $\theta=1$ and $w=0.5$ in our analysis. Parameter $\theta$ sets the threshold where $l$ is activated by $m$ and parameter $w$ gives the steepness of recruitment.

From numerical simulations when $\theta=1$, we find that when $w$ is too small, FP 3 never undergoes a Hopf bifurcation, and as $k_{l m} / k_{l}$ is increased, recurrent infection and persistent inflammation are bistable (For example, $w=0.005$ with 'healthy' parameter set in Fig. 2.2). As discussed in Appendix 2.6.1.3, small $w$ could result in a shallow enough curve (2.17) that intersects line (2.16) more than once. The two fixed points replacing FP 3 arise in a saddlenode bifurcation. The stable node is bistable with FP 5 implying that recurrent infection is bistable with persistent non-infectious inflammation. When $w$ is too large $(w=1)$, FP 3 is a stable spiral that becomes unphysical as $k_{l m} / k_{l}$ increases. Thus the system supports recurrent infection followed by persistent inflammation and healthy behavior does not exist. In summary, for $w$ too small or large the subcritical Hopf bifurcation of FP 3 (and hence healthy behavior) does not occur for any of the parameter combinations.

On keeping $w$ fixed at 0.5 and varying $\theta$, when $\theta$ is small $(\theta=0.1)$, FP 3 undergoes a supercritical Hopf bifurcation as $b$ is varied but this cannot be generically interpreted as healthy behavior as oscillations about a stable limit cycle may not always take $p$ below threshold. As $\theta$ is increased, subcritical Hopf bifurcations are possible. The range of parameter $b$, given by (2.37) and (2.38), where oscillatory behavior is observed also increases as $\theta$ is increased. Thus, large $\theta$ and moderate $w$ would maximize the region for healthy response. Figures 2.13 and 2.14 show bifurcation plots when these parameters are varied together.

In the above analysis, we have looked at varying parameter combinations rather than individual parameters for conciseness of the discussion. Varying the parameters such as $k_{p}$ and $k_{p m}$ individually does not change the bifurcation diagrams qualitatively and our interpretations of the effect of pathogen susceptibility remain unchanged. Similarly, varying $k_{l m}$ and $k_{l}$ individually does not change our conclusions about the strength of late response. The phase-space analyzed is restricted between $0<m<1$ since condition (2.17) prevents $m$ from increasing beyond one. Using any other function such as $1 /(1+m)$ to saturate $m$ can change the upper bound of $m$ but the same behavior is retained. 


\subsection{APPENDIX TO THE GLOBAL MODEL}

\subsubsection{Table of mediators modeled}

(Courtesy of Dr. G. Clermont)

\begin{tabular}{|c|c|c|}
\hline Model component & Symbol & Comment \\
\hline Pathogen & $P$ & Generic Gram negative pathogen \\
\hline Lipopolysaccharide & $P_{e}$ & Immunostimulant derived from Gram-negative bacteria \\
\hline Resting macrophage & $M_{r}$ & $\begin{array}{l}\text { Circulating monocyte or local macrophages that act } \\
\text { as a cellular pool for activated macrophages. } \\
\text { The total count of resting macrophages can } \\
\text { increase in proportion to the total inflammatory activity }\end{array}$ \\
\hline Activated macrophage & $M_{a}$ & $\begin{array}{l}\text { Activation triggered by LPS, TNF, IL-6, tissue trauma } \\
\text { and tissue dysfunction. Activation is down regulated by } \\
\text { anti-inflammatory cytokines }\end{array}$ \\
\hline Activated neutrophil & $N$ & $\begin{array}{l}\text { Activation triggered by LPS, TNF, IL-6 } \\
\text { and tissue dysfunction }\end{array}$ \\
\hline $\begin{array}{l}\text { Nitric Oxide } \\
\text { Synthase (NOS) }\end{array}$ & NOD & $\begin{array}{l}\text { Combines activities of constitutive \& inducible } \\
\text { NOS. Normally participates in blood pressure homeostasis } \\
\text { Increased by LPS, TNF in } N \& M_{a} \\
\text { Decreased by anti-inflammatory cytokines }\end{array}$ \\
\hline Circulating $\mathrm{NO}_{2} / \mathrm{NO}_{3}$ & NO & Measures correlating with cumulative NOS activity \\
\hline Tissue necrosis factor & $C_{p}$ & $\begin{array}{l}\text { Major early pro-inflammatory cytokine secreted mainly } \\
\text { by activated } M_{a} \text {, but also by } N\end{array}$ \\
\hline IL-6 & IL6 & $\begin{array}{l}\text { A pro-inflammatory cytokine with additional } \\
\text { anti-inflammatory effects }\end{array}$ \\
\hline
\end{tabular}




\begin{tabular}{|c|c|c|}
\hline $\begin{array}{l}\text { Generic anti- } \\
\text { inflammatory }\end{array}$ & $C_{a}, C_{r}$ & $\begin{array}{l}C_{a} \text { represents the combined long-lived } \\
\text { anti-inflammatory activity of IL-10, steroids, TGF- } \beta \\
\text { soluble receptors to pro-inflammatory cytokines \& intra- } \\
\text { cellular products with anti-inflammatory activity } \\
\text { such as heat-shock molecules. As a group, their } \\
\text { anti-inflammatory activity is triggered by TNF, } \\
\text { IL-6, and nitric oxide }\end{array}$ \\
\hline Activated Protein $\mathrm{C}$ & $P_{C}$ & An anti-thrombotic and anti-inflammatory agent \\
\hline Tissue factor & $T_{f}$ & Promoted by pro-inflammation, increases dysfunction \\
\hline Thrombin & $T_{H}$ & $\begin{array}{l}\text { Represents global pro-coagulant/anti-coagulant balance } \\
\text { also participate in blood pressure and dysfunction }\end{array}$ \\
\hline Blood pressure & B & $\begin{array}{l}\text { At homeostasis depends mainly on NO. The } B \text { eqn. has } \\
\text { a "restoring" term for autonomic autoregulation }\end{array}$ \\
\hline Tissue damage & $\mathrm{D}$ & $\begin{array}{l}\text { Caused by hypotension, the action of pro-inflamm. } \\
\text { cytokines, tissue microthrombosis. NO is tissue-protective } \\
\text { and there is a slow natural repair process }\end{array}$ \\
\hline
\end{tabular}

\subsubsection{Estimated values of parameters}

The 72 parameters that determine reaction rates are given below.

\begin{tabular}{|c|c|c|c|c|c|c|c|}
\hline$k_{p g} 0.1$ & $k_{p s} 0.001$ & $P_{0} 0.0001$ & $k_{p m} 0.1$ & $k_{p N O} 0.5$ & $k_{p 0_{2}} 1.0$ & $k_{p e} 0.2$ & $k_{m p} 0.5$ \\
\hline$k_{m p e} 0.02$ & $k_{m d} 0.04$ & $k_{m m} 0.1$ & $k_{m 6} 0.8$ & $k_{m r} 0.05$ & $S_{m} 0.1$ & $k_{m a} 0.2$ & $k_{n p} 0.2$ \\
\hline$k_{n p e} 1.5$ & $k_{n c p} 0.4$ & $k_{n 6} 2.0$ & $k_{n d} 0.2$ & $k_{n s} 0.1$ & $k_{n N O} 1.0$ & $k_{n O_{2}} 6.0$ & $k_{n} 0.04$ \\
\hline$S_{n} 0.001$ & $k_{N O n} 0.25$ & $k_{N O m} 0.2$ & $k_{N O 6} 0.05$ & $k_{N O D} 0.1$ & $k_{N O} 0.1$ & $k_{O 2 n} 0.2$ & $k_{O 2 m} 0.1$ \\
\hline$k_{O 26} 0.1$ & $k_{O 2 n p} 0.01$ & $k_{O 2} 0.5$ & $k_{c p n} 0.5$ & $k_{c p m r} 0.0005$ & $k_{c p m} 0.5$ & $k_{c p c p} 0.71$ & $k_{c p} 2.5$ \\
\hline$k_{6 m} 0.4$ & $k_{6 t h} 0.4$ & $k_{6 c p} 1.0$ & $k_{6} 0.1$ & $k_{c a n} 0.1$ & $k_{c a m} 0.04$ & $k_{c a c p} 0.1$ & $k_{c a 6} 0.04$ \\
\hline$k_{c a N O} 0.1$ & $k_{c a O 2} 0.1$ & $k_{c a r} 0.5$ & $k_{c a} 0.02$ & $k_{t f p e} 0.01$ & $k_{t f c p} 0.1$ & $k_{t f 6} 0.1$ & $k_{t f} 0.1$ \\
\hline$k_{t h l} 0.05$ & $k_{t h n} 0.004$ & $k_{t h} 0.1$ & $k_{p c t h} 0.1$ & $k_{p c} 0.1$ & $k_{B} 0.2$ & $k_{b n o} 0.02$ & $k_{b c p} 0.02$ \\
\hline$k_{b t h} 0.2$ & $k_{d b} 0.1$ & $k_{d c p} 0.01$ & $k_{d O 2} 0.02$ & $k_{d N O} 0.01$ & $k_{d e q} 0.1$ & $k_{d t h} 0.05$ & $k_{d} 0.03$ \\
\hline \hline
\end{tabular}


The following 20 parameters are used only in the case of specific interventions and are set to 0 in the base-line case.

\begin{tabular}{|c|c|c|c|c|}
\hline$S_{p} e$ & rate & $k_{a b}$ & $t_{a b}$ & resist \\
\hline$k_{\text {ape }}$ & $t_{\text {ape }}$ & $k_{\text {atnf }}$ & $t_{\text {iatnf }}$ & $t_{\text {fatnf }}$ \\
\hline$d u r$ & $S_{p c}$ & $t_{\text {pcon }}$ & $t_{\text {pcoff }}$ & $k_{\text {Bh }}$ \\
\hline$t_{\text {Bon }}$ & $t_{\text {Boff }}$ & $k_{d s}$ & $t_{\text {don }}$ & $t_{\text {doff }}$ \\
\hline \hline
\end{tabular}

The various thresholds of activation are as follows.

\begin{tabular}{|l|ll|ll|ll|ll|lll|ll|}
\hline$\theta 1.0$ & $\theta_{\text {cpca }} 1.0$ & $\theta_{c a} 10.0$ & $\theta_{2}$ & 10.0 & $\theta_{c p 6}$ & 1.0 & $1 \theta_{c p}$ & 1.0 & $\theta_{6}$ & 1.0 \\
\hline \hline
\end{tabular}

\subsubsection{The global model}

$$
\begin{aligned}
\frac{d P}{\tau d t}= & k_{p g} P\left(1-k_{p s} P\right) H\left(P-P_{0}\right)-k_{p m} M_{a}+k_{p N O} N O+k_{p O_{2}} O_{2}+k_{a b} H(t / \tau-t a b) \\
\frac{d P_{e}}{\tau d t}= & \left(k_{p m} M_{a}+k_{p N O} N O+k_{p O_{2}} O_{2}+k_{a b} H\left(t / \tau-t_{a b}\right)\right) P-k_{p e} P_{e} \\
\frac{d M_{r}}{\tau d t}= & -\left(k_{m p} P+k_{m p e} P_{e}+k_{m d} D\right)\left(S_{m}+f_{2}\left(C_{p}, \theta_{c p}\right)+k_{m 6} f_{2}\left(I L 6, \theta_{6}\right)\right) f_{C_{a}}\left(C_{a}, \theta_{c a}\right) M_{a} \\
& +k_{m m} f\left(C_{p}+N O+P_{e}, \theta\right)-k_{m r} M_{r}+S_{m} \\
\frac{d M_{a}}{\tau d t}= & \left(k_{m p} P+k_{m p e} P_{e}+k_{m d} D\right)\left(f_{2}\left(C_{p}, \theta_{c p}\right)+k_{m 6} f_{2}\left(I L 6, \theta_{6}\right)\right) f_{c a}\left(C_{a}, \theta_{c a}\right) M_{r}-k_{m a} M_{a} \\
\frac{d N}{\tau d t}= & \left(k_{n p} f\left(P, \theta_{2}\right)+k_{n p e} f\left(P_{e}, \theta_{2}\right)+k_{n c p} f\left(C_{p}, \theta_{c p}\right)+k_{n 6} f\left(I L 6, \theta_{6}\right)+k_{n d} f\left(D, \theta_{2}\right)\right) N\left(1-k_{n s} N\right) \\
& -\left(k_{n N O} N O+k_{n O_{2}} O_{2}\right) N-k_{n}\left(f_{s}\left(C_{p}, \theta_{c p}\right)+f_{s}\left(I L 6, \theta_{6}\right)\right) N+S_{n} \\
\frac{d N O_{d}}{\tau d t}= & \left.\left(k_{N O N} N+k_{N O M} M_{a}\right) f_{c a}\left(C_{a}, \theta_{c a}\right) f\left(C_{p}, \theta_{c p}\right)+k_{N O 6} f\left(I L 6, \theta_{6}\right)\right)-k_{N O_{d}} N O_{d} \\
\frac{d N O}{\tau d t}= & k_{N O}\left(N O_{d}-N O\right) \\
\frac{d O_{2}}{\tau d t}= & \left(\left(k_{O_{2} n} N+k_{O_{2} m} M_{a}\right)\left(f\left(C_{p}, \theta_{c p}\right)+k_{0_{2} 6} f\left(I L 6, \theta_{6}\right)\right)+k_{O_{2} n p} N f(P, \theta)\right) f_{c a}\left(C_{a}, \theta_{c a}\right)-k_{O_{2}} O_{2} \\
\frac{d C_{p}}{\tau d t}= & \left(k_{c p n} N+k_{c p m r} M_{r}+k_{c p m} M_{a}\right) f_{c a}\left(C_{a}, \theta_{c p c a}\right)\left(1+k_{c p c p} f\left(C_{p}, \theta_{c p}\right)\right) \\
& -k_{c p} C_{p}-k_{a t n f} S\left(t_{a t n f}, t_{a t n f+d u r}, \tau\right) C_{p} \\
\frac{d I L 6}{\tau d t}= & k_{6 m} M_{a}\left(1+k_{6 t h} f\left(T_{h}, \theta\right)\right) f_{c a}\left(C_{a}, \theta_{2}\right)-k_{6} I L 6
\end{aligned}
$$




$$
\begin{aligned}
\frac{d C_{r}}{\tau d t}= & \left(k_{c a n} N+k_{c a m} M_{a}\right)\left(k_{c a c p} f\left(C_{p}, \theta_{c p}\right)+k_{c a 6} f\left(I L 6, \theta_{6}\right)\right. \\
& \left.+k_{c a N O} f(N O, \theta)+k_{c a O_{2}} f\left(O_{2}, \theta\right)\right)-k_{c_{r}} c_{r} \\
\frac{d C_{a}}{\tau d t}= & C_{r}-k_{c a} C_{a} \\
\frac{d T_{f}}{\tau d t}= & \left(k_{t f p e} P_{e}+k_{t f c p} C_{p}+k_{t f 6} I L 6\right) f_{s}\left(P_{c}, \theta\right)-k_{t f} T_{f} \\
\frac{d T_{h}}{\tau d t}= & \left(k_{t h l} T_{f}+k_{t h n} T_{h}\right)-k_{t h} T_{h} \\
\frac{d P_{c}}{\tau d t}= & k_{p c t h} T_{h}-k_{p c} P_{c}+S_{p c} S\left(t_{c o n}, t_{c o f f}, \tau\right) \\
\frac{d B}{\tau d t}= & \left(k_{B}(1-B)-\left(k_{b N P} N O f_{s}\left(O_{2}, \theta\right)+k_{b c p} C_{p}+k_{b t h} T_{h}\right) B-\left(B-k_{B}\right) S\left(t_{B o n}, t_{B o f f}, \tau\right)\right. \\
\frac{d D}{\tau d t}= & \left(k_{d b}(1-B)+k_{d c p} C_{p}+k_{d O_{2}} O_{2}+k_{d N O} N O f_{s}\left(N O, \theta_{2}\right)+k_{d e q} O_{2} \exp \left(-10\left(N O-O_{2}\right)^{2}\right)\right. \\
& +k_{d t h} T_{h}-k_{d} D+k_{d s} S\left(t_{d o n}, t_{d o f f}, \tau\right)
\end{aligned}
$$

where, the functions are as follows:

$$
\begin{aligned}
f(v, \theta) & =\theta v /(v+\theta) \\
f_{2}(v, \theta) & =\theta v^{2} /\left(v^{2}+\theta\right) \\
f_{s}(v, \theta) & =\theta /(v+\theta) \\
f_{c a}(v, \theta) & =\theta /\left(v^{2}+\theta\right) \\
S\left(t_{i}, t_{f}, \tau\right) & =H\left(t / \tau-t_{i}\right)-H\left(t / \tau-t_{f}\right)
\end{aligned}
$$




\subsection{APPLICATIONS OF A GLOBAL MODEL OF ACUTE INFLAMMATION - (I) ANTHRAX AND (II) CLINICAL TRIALS}

In Section 2.3, a model of acute inflammation that incorporated some of its key features was described. Some preliminary results from it were also listed. In this chapter, some more detailed results from model simulations are discussed.

In the first section, the results from a modified version of the global model are discussed. The properties of the invading pathogen are changed to represent those of the anthrax bacterium bacillus anthracis. The typical time course of inhalational anthrax infection is reproduced. Some predictions about treatment strategies are suggested (Kumar et al., 2004a).

In the second section, an attempt is made to understand the effects of genetic diversity in a population by varying select parameters in the model (2.40). Based on such models, clinical trials to test the effectiveness of therapies could be more effectively designed (Clermont et al., 2004).

\section{$3.1 \quad$ ANTHRAX}

The history of human infection with anthrax is centuries old. More recently much has been learned about the molecular pathology of this bacterium. These developments have been spurred on by the potential and, more recently, actual use of this bacterium as a weapon of bioterror (Dixon et al., 2004). Anthrax modulates the acute inflammatory response of the host, and systemic anthrax infection is thought to result in a condition similar to sepsis and septic shock.

Three basic routes of infection are known for anthrax: cutaneous, gastrointestinal, and in- 
halational. Cutaneous anthrax is introduced into the skin through cuts or abrasions, presents as relatively nondescript skin lesions that turn a black-purplish color due to local inflammation, and resolves either on its own or with a 60-day course of antibiotics. Gastrointestinal anthrax is caused by ingestion of meat contaminated with anthrax spores, which enter the body through some break in the mucosal lining, causing fever, abdominal pain, diarrhea, constipation, or vomiting, and can result in death from perforation of the intestine or from sepsis. The third, and most lethal, form of anthrax infection is inhalational. The initial symptoms are "flu-like", caused by an exuberant inflammatory response: fever, coughing, myalgia, and malaise. The disease becomes much more severe after 1-3 days, manifesting with dyspnea, strident cough, chills, and eventually death. Though natural inhalational anthrax infection is considered rare, it is the most threatening form of anthrax infection from the point of view of bioterrorism (Shahfazand et al., 1999).

Current therapeutic strategies for inhalational anthrax are no less complex than those for sepsis and are limited in their effectiveness (Hotchkiss \& Karl, 2003).

\subsubsection{Pathogenesis of anthrax infection}

The pathogenesis of anthrax is more involved than that of a standard gram negative bacterial infection as discussed in Chapter 1. However, death from inhalational anthrax is thought to occur through the common process of systemic inflammation and bacteremia (Shahfazand et al., 1999; Abramova et al., 1993).

Anthrax infection is initiated by inhalation of aerosolized anthrax spores, which are internalized by monocytes/macrophages/dendritic cells. The spores can incubate for a period of over 40 days (modal incubation period 0-10 days) during which time monocytes and immature dendritic cells may circulate and therefore spread latent anthrax infection throughout the body. Given the appropriate temperature and/or CO2 levels (Dai \& Koehler, 1997), the spores germinate. The major toxins of B. anthracis are protective antigen, lethal factor, and edema factor. These three exotoxins are synthesized and each form heterodimers that result in bioactive molecules: edema toxin is a heterodimer of protective antigen and edema factor, and lethal toxin consists of protective antigen bound to lethal factor (Shahfazand 
et al., 1999; Dixon et al., 2004).

Edema factor is a calmodulin-dependent adenylate cyclase, which causes elevations of the intra-cellular second messenger cyclic adenosine mono phosphate (cAMP) (O'Brien et al., 1985). Edema toxin attracts neutrophils to the site of infection, but suppresses the capacity of these cells to phagocytose bacteria and to produce super-oxide in response to this phagocytosis.

Lethal factor, a zinc metalloprotease, inactivates members of the mitogen-activated protein kinase family of signaling molecules, known to be involved in the responses to stress and inflammation (Bonventre, 2003). In vitro evidence shows that lethal toxin in small doses stimulates macrophages to produce IL- $1 \beta$ and TNF- $\alpha$ and in large doses causes lysis of macrophages (Friedlander, 1986). In the process of macrophage death, it has been hypothesized that there is an apparent rapid and overwhelming release of pro-inflammatory cytokines which is thought to result in the 'sudden death' seen in inhalational anthrax. Thus lethal toxin through a macrophage dependent process is thought to be the principal cause of death in anthrax infection (Hanna et al., 1993).

Protective antigen plays the important role of allowing intra-cellular entry of the other two factors by binding to them. Even though it has no direct toxic effects, strains of bacteria deficient in protective antigen production are far less virulent than the wild type bacterium (Pezard et al., 1995; Brossier et al., 2000).

However, there has been evidence recently that suggests that mortality due to anthrax is due to non-inflammatory mechanisms and due to hypoxia-induced liver failure where an Fas-L dependent process has been implicated (Prince, 2003). Mice injected with anthrax lethal toxin intra peritoneally have revealed no evidence of TNF production or symptoms of systemic inflammation. Tissue hypoxia, liver necrosis and pleural edema and an initial spike of IL-1 $\beta$ concomitant with macrophage lysis were observed (Moayeri et al., 2003). However, these observations were based on i.p. injections of anthrax toxins and pathogenesis of such a gastro-intestinal disease might well be different from that of inhalational anthrax infection(Abramova et al., 1993).

The virulence of anthrax is enhanced by yet another factor. The vegetative bacteria is enclosed in a anti-phagocytic poly-D-glutamic acid capsule. This makes the bacteria more 
difficult to phagocytose (Hanna et al., 1993). In fact, non-encapsulated versions of the strain are attenuated enough that they are routinely used as vaccines in animals (Schneerson et al., 2003).

\subsubsection{Simulations from a model of anthrax infection}

The equations (2.40) model the acute inflammatory response to a general gram negative bacterium. This is modified to simulate the typical course of an anthrax infection by introducing equations describing the specific properties of anthrax bacterium. Based on the hypotheses of the pathogenesis, a variable for the sterile spore is introduced which gets ingested by patrolling resting macrophages. The macrophage is activated and the bacteria start growing and these processes are modeled using the following equations.

$$
\begin{aligned}
\frac{d S P O R E}{\tau d t}= & -k_{s p} \text { SPORE * } M_{r} \\
\frac{d P}{\tau d t}= & k_{p g} P\left(M_{r}+M_{a}-k_{p s} P\right) H\left(P-P_{0}\right) \\
& -\left(k_{p m} M_{a}+k_{p N O} N O+K_{p O_{2}} O_{2}+k_{a b} H\left(t-t_{a b}\right)\right) P+k_{s p} S P O R E M(3) \\
\frac{d M_{a}}{\tau d t}= & \left.k_{m p} P+k_{m d} D\right)\left(f_{2}\left(C_{p}, \theta_{c p}\right)+K_{m 6} f_{2}\left(I L 6, \theta_{6}\right)\right) \\
& f_{c a}\left(C_{a}, \theta_{c a}\right) M_{r}-k_{m a} M_{a}+K_{m l t}\left(L_{T} M_{r}-L_{T}^{2} M_{a}\right)+k_{m s p} S P O R E M_{r}
\end{aligned}
$$

The growing bacteria releases the edema factor, lethal factor and protective antigen which are modeled as follows.

$$
\begin{aligned}
\frac{d E_{F}}{\tau d t} & =k_{e f p} P-k_{e f} E_{F} \\
\frac{d L_{F}}{\tau d t} & =k_{l f p} P-k_{l f} L_{F} \\
\frac{d P_{A F}}{\tau d t} & =k_{p a f p} P-k_{p a f} P_{A F}
\end{aligned}
$$


Edema factor combines with protective antigen to form edema toxin and lethal factor similarly forms lethal toxin as follows.

$$
\begin{aligned}
\frac{d E_{T}}{\tau d t} & =k_{e p a f} E_{F} P_{A F}-k_{e t} E_{T} \\
\frac{d L_{T}}{\tau d t} & =k_{\text {lpaf }} L_{F} P_{A F}-k_{l t} L T
\end{aligned}
$$

The effects of these toxins are modeled by modifying the previously existing equations. Lethal toxin gets absorbed by resting macrophages and in large quantities causes its lysis and massive release of TNF. This is reflected as follows in the equations for TNF.

$$
\begin{aligned}
\frac{d C_{p}}{\tau d t}= & \left(k_{c p n} N+k_{c p m r} M_{r}+k_{c p m} M_{a}\right) f_{c a}\left(C_{a}, \theta_{c p c a}\right)\left(1+k_{c p c p} f\left(C_{p}, \theta_{c p}\right)\right)+k_{c p B O M B}\left(k_{m l t} M_{a} L_{T}^{2}\right) \\
& -k_{c p} C_{p}-k_{a t n f} S\left(t_{a t n f}, t_{a t n f+d u r}, \tau\right) C_{p}
\end{aligned}
$$

Edema toxin increases concentration of neutrophils but decreases their capacity to produce O2 and NO which are the chemicals that are actually involved in phagocytosis as shown below.

$$
\begin{aligned}
\frac{d N}{\tau d t}= & \left(k_{n e t} E_{T}+k_{n p} f\left(P, \theta_{2}\right)+k_{n c p} f\left(C_{p}, \theta_{c p}\right)+k_{n 6} f\left(I L 6, \theta_{6}\right)+k_{n d} f\left(D, \theta_{2}\right)\right) N\left(1-k_{n s} N\right) \\
& -\left(k_{n n o} N O+k_{N_{2}} O_{2}\right) n-k_{n}\left(f_{S}\left(C_{p}, \theta_{c p}\right)+f_{s}\left(I L 6, \theta_{6}\right)\right) N+S_{n} \\
\frac{d O_{2}}{\tau d t}= & \left(\frac{k_{O_{2} n} N}{1+E_{T}}+k_{O_{2} m} M_{a}\right)\left(f\left(C_{p}, \theta_{c p}\right)+k_{0_{2} 6} f\left(i l 6, \theta_{6}\right)\right) \\
& \left.\left.+k_{O_{2} n p} n f(P, \theta)\right) f_{c a}\left(C_{a}, \theta_{c a}\right)-k_{O_{2}} O_{2}\right)
\end{aligned}
$$

The anthrax bacteria is more difficult to phagocytose than a gram-negative bacteria because of its capsule. Thus, anthrax infection leads to shock and large bacterial counts systemically. These changes to the model of gram-negative sepsis are summarized in Appendix 3.3. However, the model's immune parameters that were fixed using the model (2.40) were not modified.

Simulations from the modified model for anthrax infection are shown in Figure 3.1 and can be compared with the sepsis simulations from a generic gram negative bacterium in Figure 2.16. Anthrax-mediated tissue damage/dysfunction (representative of organ damage 
and, presumably, lethality) remains elevated for several hours. Bacteria remain uncleared despite high levels of inflammation. (In order to offer an unbiased comparison between Gramnegative infection and anthrax, the number as well as intrinsic growth and death parameters of all bacteria are kept the same.) Thus severe septic shock is the outcome of infection with anthrax bacterium at an inoculum that is non-lethal in the case of regular gram negative bacteria.

\subsubsection{Treatment and prevention strategies: Current status}

Below some of the most frequently used treatment strategies against anthrax are examined. Though several have been developed to deal with the epidemic in animals, vaccination data from humans is understandably difficult to come by. Analyzing the results from mathematical models like these provides clinicians with vital clues about administration of therapies.

One of the most common treatment methods for all infections, including anthrax, is antibiotic administration. Antibiotics may work for cutaneous and gastro-intestinal anthrax. However, it has been widely accepted that by the time the symptoms of inhalation anthrax are patent, it is too late for anti-microbial therapy to be effectual (Shahfazand et al., 1999; Dixon et al., 2004).

Various types of vaccines have been suggested to be used in animals and susceptible human populations. "Live" vaccines are preparations made from naturally occurring attenuated bacteria or genetically modified versions which are less virulent. Injecting such vaccines has been shown to effectively protect most animals from anthrax. However, inherent risks of live bacterial injections are associated with these vaccines (Pezard et al., 1995).

Consequently, recent vaccines prepared for use in humans consist of "cell-free" filtrates of the bacilli produced to increase the content of protective antigen (PA). The preparation is then alum-precipitated to form the vaccine. There are few human studies of effectiveness of such vaccines. In a single-blind placebo controlled study based on tannery workers, the vaccine was found to be $92.5 \%$ effective in preventing anthrax. However, this included cases of cutaneous anthrax and protection from the more serious inhalational anthrax could not be assessed (Shahfazand et al., 1999). 
Thus effective and safe treatment and prevention strategies against inhalational anthrax for susceptible human populations have not been determined and possible vaccine mechanisms are investigated using the mathematical model.

\subsubsection{Predictions from the model}

Vaccination alone or in combination with antibiotic administration in the setting of an anthrax infection has been simulated. An antibiotic was simulated as a chemical that would kill live bacteria on contact. The vaccine was simulated by changing the parameters of production of Protective Antigen $\left(k_{p a f p}\right.$ and $\left.k_{p a f}\right)$ to result in reduced amounts of available Protective Antigen.

3.1.4.1 Vaccine against protective antigen alone may protect The model suggests that effective vaccination against protective antigen results in tissue dysfunction that is somewhat mitigated. This is not immediately interpretable as "dead" or "alive". Though, tissue dysfunction may be low, pathogen load remains high. This is because in the vaccinated individual, protective antigen which is one of the key stimulators of the immune response, is down-regulated. This could result in uncontrolled bacterial growth which could also lead to death (Fig. 3.2).

The results that have been presented so far are of a single set of genetic parameters. However, the outcome of an infection depends on several factors including the virulence of invading pathogen, the size of the inoculum, and the patients coexisting conditions, age, and gene polymorphisms (Hotchkiss \& Karl, 2003). Varying the response of the macrophages and neutrophils and early cytokines $\left(k_{m p}, k_{n p}, k_{c p n}\right.$ etc.), varies the effectiveness of the vaccine. There are reasonable parameter values where the administration of protective antigen alone is sufficient in restoring health (Fig. 3.4).

Based on model simulations, we can predict that the strategy of using anti-protective vaccine alone can work for some individuals. When circulating neutrophils respond strongly to the bacterial coat, it effectively counteracts the immune suppression caused by smaller amounts of protective antigen. Increased production of NO aids in clearing bacteria and 
does not cause damage downstream. However, a strong macrophage response is confounding as, based on our hypotheses, it could result in large amounts of circulating cytokines which could lead to septic shock.

3.1.4.2 Combination therapy is the most ideal In Fig. 3.3, a simulation of the predicted efficacy of pre-existing antibodies to protective antigen, in the presence or absence of antibiotics is shown. This simulation is of one set of genetic parameters. In all cases, tissue dysfunction is shown: rising tissue dysfunction is equated with death, while dysfunction that returns to baseline is synonymous with survival. Anthrax infection by itself is simulated first (Case 1; Fig. 3.3), and this would results in rising tissue dysfunction that is interpreted as a negative outcome. The administration of antibiotics at $\mathrm{t}=0$ (Case 2; Fig. 3.3; an unlikely scenario in an anthrax outbreak) resolves the infection and results in survival (falling tissue dysfunction). The administration of antibiotics at $\mathrm{t}=48 \mathrm{~h}$ (Case 3; Fig. 3.3; a likely clinical scenario in an anthrax outbreak) results in death (rising tissue dysfunction) essentially identical to that of Case 1, again in agreement with published results showing lack of efficacy of late antibiotic administration in anthrax-infected mice (Kalns et al., 2002). The presence of pre-existing circulating antibodies to protective antigen (Case 4; Fig. 3.3; the anthrax vaccine case) results in reduced dysfunction as compared to Cases 1 or 3, but this effect is not as dramatic as that observed in Case 2. This result is not immediately classifiable as death as discussed above. However, if a patient has pre-existing circulating antibodies to protective antigen and receives antibiotics at $\mathrm{t}=48 \mathrm{~h}$ (Case 5; Fig. 3.3; a case representing a vaccinated individual that is infected with anthrax), improvement is seen relative to Cases 1, 3, and 4 (but not Case 2). Thus, the model predicts that a combination of pre-existing circulating antibodies to protective antigen and timely administration of antibiotics is the vaccine is a realistic and effective strategy to deal with sepsis caused by anthrax. This combination strategy is supported by experimental evidence in monkeys (Friedlander et al., 1993). 

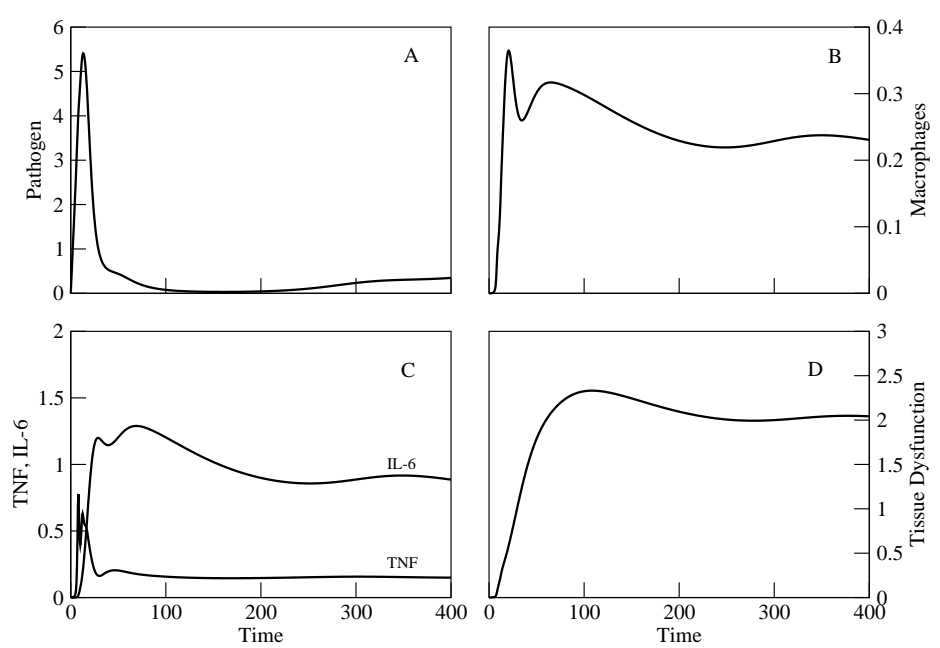

Figure 3.1: Modification of mathematical model of sepsis to account for anthrax-specific effects: Comparison of the predicted effect on tissue damage/dysfunction of release of proinflammatory cytokines in anthrax infection. We observe that despite an exuberant immune response the bacterial load remains high. (The bacterial growth parameters are the same as that of the gram-negative infection in Fig. 2.15) 

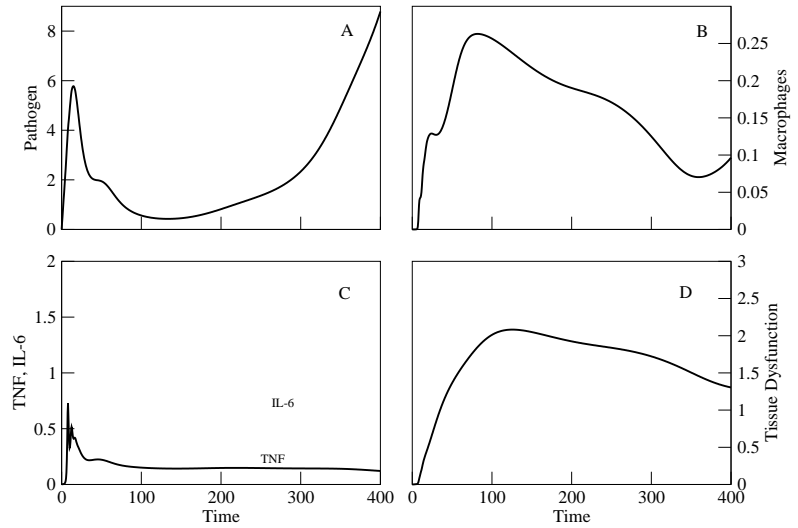

Figure 3.2: Vaccination against protective antigen leads to a decrease in the stimulation of the immune response. The primary stimulants are now the bacterial coats and that is not enough to clear bacteria. Therefore, though a down-regulated immune response leads to lower damage, it also results in unbounded growth of bacteria

\subsubsection{Discussion of the anthrax model}

The approach to the problem of anthrax infection in the above argument, is to consider the sequelae of inhalational anthrax exposure to be a specialized form of septic shock, and to model mathematically the response to infection. The mathematical model of general acute inflammatory response to gram negative bacteria (2.40) has been modified (3.3) to capture features specific to anthrax infection, including the anti-phagocytic capsule, the synthesis of edema toxin and lethal toxin from their components, chemoattraction of neutrophils and subsequent suppression of their oxidative burst, and activation and subsequent destruction of macrophages. Anthrax infection simulated with the above properties results in septic shock with increased levels of cytokines, damage, decreased blood pressure and uncleared bacterial load and is interpreted as death.

This model simulates the efficacy of antibiotic administration. As observed in animal experiments, the model suggests that antibiotics administered early in the course of infection are useful in clearing it and restoring homeostasis. Late administration of antibiotics is not 


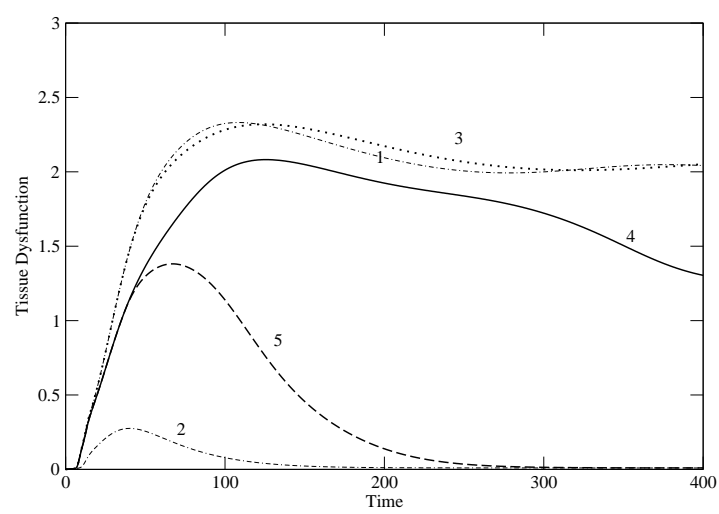

Figure 3.3: Simulation of the efficacy of antibiotics and vaccination in inhalational anthrax: Simulation of tissue damage/dysfunction under the various circumstances. Case 1, anthrax infection, no antibiotics. Case 2, anthrax infection, antibiotic administration at $\mathrm{t}=0$. Case 3 , anthrax infection, antibiotic administration at $\mathrm{t}=48 \mathrm{~h}$. Case 4 , anthrax infection in the setting of vaccination leading to pre-existing circulating antibodies to protective antigen. Case 5, anthrax infection in the presence of vaccination and antibiotic administration at $\mathrm{t}$ $=48 \mathrm{~h}$ 


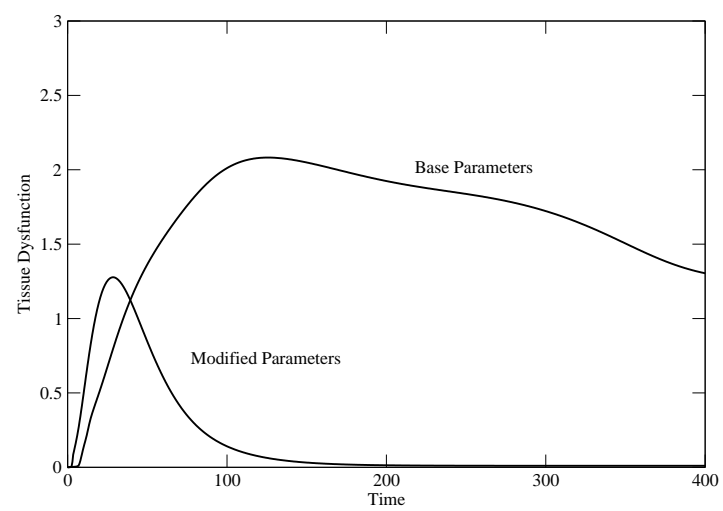

Figure 3.4: Simulation of a set of parameters where vaccination alone is sufficient in curing $\operatorname{anthrax}\left(k_{p a f p}\right.$ is set $=0.001$; its base value $\left.=0.015\right)$ : Varying the parameters in the model could resemble the inherent variations in a population. In the above simulation, pathogen and vaccine properties are kept the same, but immune response parameters are changed. $k_{n p}=4$ ( 8 times base value), $k_{N O n}=3$ ( 6 times base value). Tissue dysfunction, in the presence of anti-protective antigen vaccine, in the case of the base parameter values is compared with the modified set. 
useful as it cannot alter the course of the infection (Kalns et al., 2002).

The effect of administering vaccines against protective antigen can also be tested in the model. Experimental evidence about the efficacy of the vaccine in humans is sparse. We suggest that vaccines on their own may be effective in clearing anthrax infection only in some cases. Clearing protective antigen implies less of a stimulation to the immune system. The model suggests that in a generic case, this could lead to a slow immune response which grows with the bacteria and is ultimately inadequate in clearing it. However, individuals with strong initial immune response to the bacterial coat and a muted late inflammatory wave may be cured with vaccination alone.

The presence of protective antigen in the sequence of anthrax infection incites a strong pro-inflammatory response in the body pre-disposing it to tissue damage and eventually septic shock. Therefore, clearing protective antigen using the vaccine has an anti-inflammatory effect systemically. Given favorable circumstances, such as low virulence pathogen, well calibrated dosage of vaccine, administration of the vaccine alone could be enough to take the system to health. However, in the scenario of depleted protective antigen, the main instigator of the immune response is the growing pathogen alone and chances of an inadequate immune response resulting in persistent infection and subsequent systemic inflammation are high. Late antibiotics actually help in clearing pathogen and restoring the system to health. Hence a combination therapy to down-regulate inflammation and to later clear bacteria is likely to be successful for a large population.

\subsection{CLINICAL TRIALS}

In this section, the effectiveness of anti-TNF- $\alpha$ therapy in preventing sepsis in a population is explored using the model (2.40). As discussed in Chapter 1, TNF- $\alpha$ is one of the key mediators in the acute inflammatory response and could play a major role in taking a system to sepsis. Supported by data from animal experiments, it was believed that targeting to down-regulate TNF- $\alpha$ in a septic patient was likely to alleviate the condition. Large randomized clinical trials were conducted to test the hypothesis and the therapy failed to meet the criteria for efficacy and was not approved (Reinhart \& Karzai, 2001; Abraham et al., 
1998).

In this analysis, we attempt to carry this out "in silico" to gain insight into the actual effectiveness of the therapy. A protocol to best simulate the setting of a clinical trial is designed. The model generates information on the possible biological markers, the genetic profile of those benefitted and harmed by the therapy and the best timing and dosage for its administration. The complete analysis is available in (Clermont et al., 2004).

The behavior of a system with varying parameters can be understood, in principle, through a complete bifurcation analysis. But in the case of complex models, such a systematic analysis is likely to be very complicated. Instead, in the following study, only the parameter regimes of interest are probed in parts ("randomization") and some useful conclusions are drawn.

\subsubsection{Design of the clinical trial}

The clinical trial was designed to focus on the consequences of the administration of a neutralizing antibody directed against the pro-inflammatory cytokine tumor necrosis factor (anti-TNF) to sepsis patients (Reinhart \& Karzai, 2001). This was simulated in the model by adding a term to reduce the amount of TNF- $\alpha$ and the strength of this clearance is controlled by the parameter $k_{a t n f}$. This clearance is implemented by a Heaviside function between times $t_{i}$ and $t_{f}$ (see model 2.40).

The effect of anti-TNF therapy is evaluated by administering to a genetically diverse population, that is exposed to different insults. A population of 1000 virtual patients differing in their initial bacterial load $(p(0))$, bacterial virulence (bacterial growth parameter $k_{p g}$ and time of initiation of intervention $\left(t_{i}\right)$ are generated. Pathogen characteristics (growth rate and initial load) were chosen to result in a survival of approximately $60 \%$. The delay before medical consultation, and thus time of initiation of treatment was variable and depended on pathogen load and virulence (i.e. sicker cases would generally consult earlier).

To simulate genetic diversity of the study population, individual propensity of immune cells to generate effector molecules (pro-inflammatory [TNF and IL-6], anti-inflammatory $[\mathrm{CA}]$, and nitric oxide [NO] activity) was randomly varied between $\pm 25 \%$ of baseline param- 
eters as dictated by literature data (Louis et al., 1998). Those variations were sufficient to explain wide swings in individual serum levels of effectors.

To identify optimal dosing and duration of administration strategies, the virtual cohort of 1000 patients were treated with nine different interventions of anti-TNF. The duration of administration of anti-TNF $\left(6 \mathrm{~h}, 24 \mathrm{~h}\right.$, or $48 \mathrm{~h}$ ) was varied (i.e. $t_{f}-t_{i}$ ). Comparatively, the half-life of anti-TNF antibodies in naive patients is 40 to 50 hours (Fisher et al., 1993). Three different "doses" of anti-TNF $\left(2,10\right.$, and 20 arbitrary units) were used (i.e. $\left.k_{a t n f}\right)$. Depending on dose, TNF neutralization varied from $18.6 \%$ to $55.5 \%$ of total TNF produced in controls. It is difficult to obtain a clear correlation with published reports as these do not typically report areas under the curve, and do not always distinguish between biologically active TNF, TNF bound by antibody and TNF bound by specific soluble receptors (Fisher et al., 1993).

\subsubsection{Results and Discussion}

The simulations clearly separate cases with favorable and unfavorable outcomes based on global tissue dysfunction as shown in Figure 3.5. Overall mortality was $37.1 \%$ as determined by tissue dysfunction at 168 hours divided by maximal tissue dysfunction at any point during the first 168 hours. This variable is chosen as a proxy to unfavorable outcome because there was a clear bimodal distribution of this variable after 72 to 96 hours. Therefore, the model offered a natural separation of favorable and unfavorable outcomes.

There is a logical physiological correlate to this observation: after reaching a maximum, tissue dysfunction tended to improve significantly and rapidly in some cases (survivors), or remained elevated at a substantial fraction of maximum in all other cases (non-survivors). Mortality was higher with high bacterial load, high virulence, and high TNF production potential by effector cells.

Depending on dose and duration of treatment, survival ranged from $57.1 \%$ to $80.8 \%$. Higher doses of anti-TNF, although effective, also resulted in considerable harm to patients. Trial optimization involves selecting a dosing strategy that optimizes outcome in a cohort of patients, and then selecting patients that would benefit from treatment while avoiding 
treating patients for which treatment would have either no effect or cause harm.

A saturation of the effect of anti-TNF on peak and cumulative concentration of mediators and tissue dysfunction was observed. Survival is highest when the lowest dose of anti-TNF is administered for 48 hours, and worst when the highest dose is administered for 48 hours. A surprising, yet key, finding is that anti-TNF treatment helps a significant percentage of individuals, but also harms many. Thus, the beneficial effect of this therapy is reduced considerably if administered in a randomized fashion.

It is also possible to identify subgroups with high propensity for being helped or harmed by the proposed intervention, and identify early serum markers for each of those subgroups (see (Clermont et al., 2004)).

The relationship between gene polymorphism and outcome of patients with sepsis has been difficult to document (Louis et al., 1998). Although models such as the one presented provide clear predictions, these are difficult to confirm in the absence of consistent clinical and epidemiological studies. 


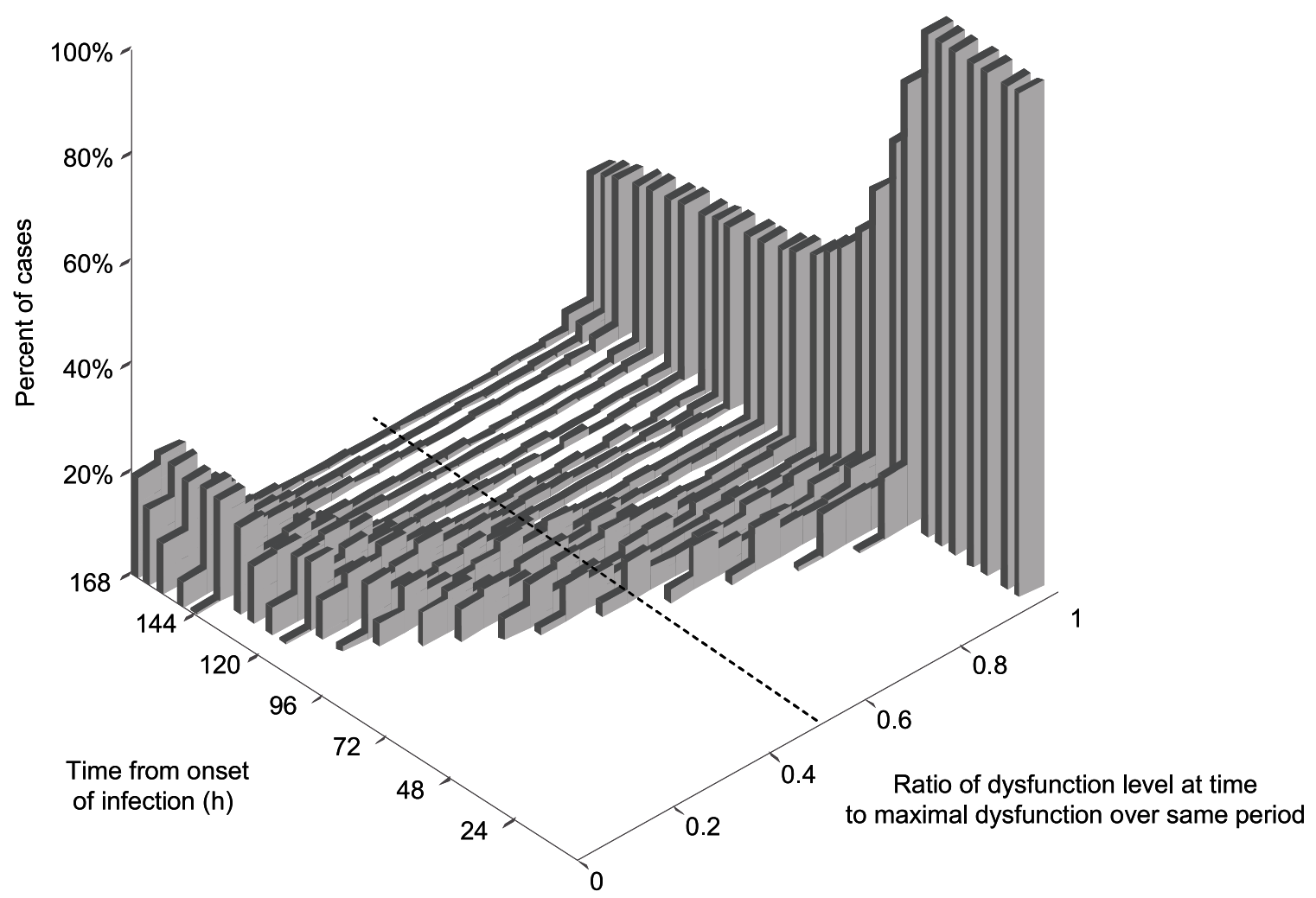

Figure 3.5: The clear separation of positive and negative outcomes in terms of tissue dysfunction in a randomized clinical trial. The axis marked from 0 to 168 is the time from onset of infection. The axis marked from 0 to 1 shows the ratio of dysfunction at that time to maximal dysfunction. (Courtesy of Dr.G.Clermont) 


\subsection{APPENDIX: MODIFICATION OF THE DETAILED MODEL TO SIMULATE ANTHRAX INFECTION}

The general model (2.40) is modified to simulate anthrax infection, by removing the equation for endotoxin pe and adding the following equations for the anthrax toxins. The mechanism for the spore getting converted to active bacterium is also modeled. The susceptibility parameters of the pathogen are decreased in comparison with (2.40) to model the antiphagocytic capsule. The effects of the toxins are captured by changing the equations for neutrophils and TNF- $\alpha$ (represented by $C_{p}$ in the model). Only the equations that have been modified in some manner from (2.40) are presented here.

$$
\begin{aligned}
\frac{d S P O R E}{\tau d t}= & -k_{s p} S P O R E * M_{r} \\
\frac{d P}{\tau d t}= & k_{p g} P\left(M_{r}+M_{a}-k_{p s} P\right) H\left(P-P_{0}\right) \\
& -\left(k_{p m} M_{a}+k_{p N O} N O+K_{p O_{2}} O_{2}+k_{a b} H\left(t-t_{a b}\right)\right) P+k_{s p} S P O R E M_{r} \\
\frac{d E_{F}}{\tau d t}= & k_{e f p} P-k_{e f} E_{F} \\
\frac{d L_{F}}{\tau d t}= & k_{l f p} P-k_{l f} L_{F} \\
\frac{d P_{A F}}{\tau d t}= & k_{p a f p} P-k_{p a f} P_{A F} \\
\frac{d E_{T}}{\tau d t}= & k_{e p a f} E_{F} P_{A F}-k_{e t} E_{T} \\
\frac{d L_{T}}{\tau d t}= & k_{l p a f} L_{F} P_{A F}-k_{l t} L T \\
\frac{d M_{a}}{\tau d t}= & \left.k_{m p} P+k_{m d} D\right)\left(f_{2}\left(C_{p}, \theta_{c p}\right)+K_{m 6} f_{2}\left(I L 6, \theta_{6}\right)\right) \\
& f_{c a}\left(C_{a}, \theta_{c a}\right) M_{r}-k_{m a} M_{a}+K_{m l t}\left(L_{T} M_{r}-L_{T}^{2} M_{a}\right)+k_{m s p} S P O R E M_{r} \\
\frac{d N}{\tau d t}= & \left(k_{n e t} E_{T}+k_{n p} f\left(P, \theta_{2}\right)+k_{n c p} f\left(C_{p}, \theta_{c p}\right)+k_{n 6} f\left(I L 6, \theta_{6}\right)+k_{n d} f\left(D, \theta_{2}\right)\right) N\left(1-k_{n s} N\right) \\
& -\left(k_{n n o} N O+k_{N O_{2}} O_{2}\right) n-k_{n}\left(f_{S}\left(C_{p}, \theta_{c p}\right)+f_{s}\left(I L 6, \theta_{6}\right)\right) N+S_{n} \\
\frac{d O_{2}}{\tau d t}= & \left(\frac{k_{O_{2} n} N}{1+E_{T}}+k_{O_{2} m} M_{a}\right)\left(f\left(C_{p}, \theta_{c p}\right)+k_{0_{2} 6} f\left(i l 6, \theta_{6}\right)\right) \\
& \left.\left.+k_{O_{2} n p} n f(P, \theta)\right) f_{c a}\left(C_{a}, \theta_{c a}\right)-k_{O_{2}} O_{2}\right) \\
\frac{d C_{p}}{\tau d t}= & \left(k_{c p n} N+k_{c p m r} M_{r}+k_{c p m} M_{a}\right) f_{c a}\left(C_{a}, \theta_{c p c a}\right)\left(1+k_{c p c p} f\left(C_{p}, \theta_{c p}\right)\right)+k_{c p B O M B}\left(k_{m l t} M_{a} L_{T}^{2}\right) \\
& \left.-t_{a t n f}, t_{a t n f+d u r}, \tau\right) C_{p}
\end{aligned}
$$




\subsubsection{Parameters varied}

The rate constants that are varied from the model (2.40) are as follows.

\begin{tabular}{|c|c|c|c|}
\hline$k_{\text {pпo }} 0.2$ & $k_{p O_{2}} 0.2$ & $k_{p m} 0.2$ & $k_{\text {epaf }} 1.0$ \\
\hline$k_{\text {efp }} 0.15$ & $k_{\text {ef }} 0.5$ & $k_{\text {lfp }} 0.15$ & $k_{\text {et }} 0.5$ \\
\hline$k_{l f} 0.5$ & $k_{\text {pafp }} 0.015$ & $k_{\text {paf }} 0.5$ & $k_{\text {lpaf }} 1.0$ \\
\hline$k_{l t} 0.5$ & $k_{\text {mlt }} 0.05$ & $k_{\text {net }} 0.5$ & $k_{\text {cpbomb }}=1.0$ \\
\hline
\end{tabular}




\subsection{THE THRESHOLD OF EXTINCTION OF DECAYING POPULATIONS IN A DETERMINISTIC MODEL}

Ordinary Differential Equations (ODEs) are often used to model interacting populations. In such models, deterministic behavior of continuous populations and spatial homogeneity are assumed. Although powerful results may be obtained from the analysis of ODE models, models that explicitly account for stochasticity and spatial distribution could result in qualitatively different and often more fundamental understanding of the systems studied (Durrett \& Levin, 1994; Nasell, 1995; Gandhi et al., 1998).

In particular, the extinction of a population cannot be handled using differential equations. In the ODE models of acute inflammation discussed in Chapter 2 immune-cells respond to an invading pathogen and attempt to eliminate it in a manner similar to a predator-prey model (Kumar et al., 2004b). While the pathogen population can diminish exponentially fast when acted on by the immune-cells, it can never become completely eliminated in a finite time. Thus a consequence is that if the immune-cells return to rest after an inflammatory episode, then the pathogen will re-grow from an infinitesimal quantity. This is an artifact that arises because the approximation of pathogen population as a continuous variable breaks down when the population becomes small and the discreteness of the pathogen number becomes important. In the continuous, deterministic model, populations never go to 0 and thus unstable cycles are seen in the phase-plane. However, in a discrete, stochastic model, the cycles are not expected to be sustained, as fluctuations will take the populations to zero after which there is no possibility for further dynamics. Thus, in this regime, a stochastic or agent based model where the pathogen can be completely eliminated is more appropriate. This problem is encountered in several ecological and other population models and is usually handled by introducing an arbitrary low threshold for the population in the 
ODE, below which it is considered to be extinct (Williams \& Martinez, 2004; Abramson \& Zanette, 1998; Yodzis \& Innes, 1992). Several studies have attempted to estimate times to extinction in population models and a detailed survey of the various approaches can be found in Nasell (2001). The earliest and most fundamental work (Bartlett, 1966) examines the probability distribution of a population given a birth and death rate, and estimates the chance to extinction based on parameters in such a probabilistic model. Another study (Solari \& Natiello, 2003) approximates the probability distribution of a population model with a product of probabilities of independent Poisson processes and then predicts the times to extinction and other quantities of interest. A fully probabilistic 2-dimensional model of an epidemic of infection is simplified using conditional probability distributions and the time to extinction can be predicted (Nasell, 1999; Nasell, 2001). Nasell (1999), also compares the time to extinction with previous predictions.

In this chapter, the average time to extinction for a decaying population is estimated from the probabilistic analysis of a simple 1-dimensional decay (with varying decay rates). Using this time, an effective threshold is obtained below which the pathogen is assumed to be extinct. The goal is to retain the ODE description to the limit of its validity and terminate it as the description breaks down at very small numbers. The effectiveness of this threshold in deterministic models of interacting populations is also examined. A Fokker-Planck estimate for a threshold is also derived and found to be unsatisfactory for this problem, as suggested by other studies (Doering \& Sander, 2004). Spatial homogeneity is assumed in this analysis and further corrections may be necessary to understand extinction in interacting populations.

\subsection{THRESHOLD FOR THE GENERAL DECAY PROCESS FROM THE MASTER EQUATION AND GILLESPIE SIMULATION}

Consider a general decay process represented by :

$$
x+1 \stackrel{k(t)}{\rightarrow} x
$$

where

$$
k(t) \geq 0 \forall t
$$


Modeling this process by the differential equation $d x / d t=-k(t) x$, yields the solution

$$
x(t)=x_{0} \exp (-(g(t)-g(0)))
$$

where $g(t)=\int_{0}^{t} k\left(t^{\prime}\right) d t^{\prime}$. In such a model $x$ is thought to represent the average of a large number of ensembles and $x$ is approximated by a continuous variable. This solution suggests that the average of an ensemble goes to zero when $t \rightarrow \infty$.

However, a more accurate representation is to consider the population $x$ to be discrete and the decay to be a stochastic process. Then, in principle, the statistics of the decay process can be obtained from solving its Master equation.

We propose that the average time at which a population decays can be found from such a discrete, stochastic model and a threshold can be computed from it that can be used as cut-off in the continuous, deterministic model.

The Master equation for the probability distribution of $x$ in time $t$ for the general decay process is as follows

$$
\frac{d P(x, t)}{d t}=k(t)[(x+1) P(x+1, t)-x P(x, t)]
$$

The above equation is discrete in $x$ and continuous in $t$. The solution to this equation can be found exactly using an iterative process and induction (Appendix 4.4.1) with the condition that $P(x, 0)=\delta\left(x, x_{0}\right)$ where $x_{0}$ is the initial population. The solution is given by

$$
P(x, t)=\left(\begin{array}{c}
x_{0} \\
x
\end{array}\right) \exp \left(-\left(g(t)-g_{0}\right)\right)^{x}\left(1-\exp \left(-\left(g(t)-g_{0}\right)\right)^{\left(x_{0}-x\right)}\right) .
$$

Now the average time this system takes to decay can be calculated as follows. $P(0, t)$ is the probability of the population being 0 at time $t$ and from Equation 4.5, is given by

$$
P(0, t)=\left(1-\exp \left(-\left(g(t)-g_{0}\right)\right)\right)^{x_{0}} .
$$

Then, we define the probability density $\Pi(0, t)$ that describes the fraction of particles that go from state ' 1 ' to state ' 0 ' between time $t$ and $t+d t$ as

$$
\frac{d P(0, t)}{d t}=\Pi(0, t)=k(t) x_{0} \exp \left(-\left(g(t)-g_{0}\right)\right)\left(1-\exp \left(-\left(g(t)-g_{0}\right)\right)\right)^{x_{0}-1}
$$


with the following properties: $\int_{t_{1}}^{t_{2}} \Pi(t) d t$ is the fraction of particles that decay in the time interval $\left[t_{1}, t_{2}\right] ; \int_{0}^{\infty} \Pi(t) d t \equiv 1$ because all the particles are expected to have decayed in infinite time. In order for this normalization to be valid, we insist that

$$
g(\infty)=\infty
$$

So the average time $\langle T\rangle$ it takes for the particles to decay is given by the integral,

$$
\int_{0}^{\infty} t \Pi(t) d t=\langle T\rangle
$$

This can be solved by expanding $\left(1-\exp \left(-\left(g(t)-g_{0}\right)\right)\right)^{x_{0}}$ in the integral as a binomial series (Appendix 4.4.1) and $\langle T\rangle$ is given as

$$
\langle T\rangle=x_{0} \sum_{j=0}^{x_{0}-1} \frac{(-1)^{j}}{j+1}\left(\begin{array}{c}
x_{0}-1 \\
j
\end{array}\right) \int_{0}^{\infty} \exp \left(-(j+1)\left(g(t)-g_{0}\right)\right) d t
$$

Similarly an expression can be found for $\left\langle T^{2}\right\rangle$ from which the variance can be computed.

$$
\left\langle T^{2}\right\rangle=2 x_{0} \sum_{j=0}^{x_{0}-1} \frac{(-1)^{j}}{j+1}\left(\begin{array}{c}
x_{0}-1 \\
j
\end{array}\right) \int_{0}^{\infty} t \exp \left(-(j+1)\left(g(t)-g_{0}\right)\right) d t
$$

(The integral for the average time (4.7) may also be solved in an asymptotic expansion in order to more easily determine the behavior as $x \rightarrow \infty$ (Kumar \& Chow, 2004). For $k(t)=1,\left\langle T\left(x_{0}=\infty\right)\right\rangle \rightarrow$ Euler's constant $\left.(0.5772 .).\right)$ Thus the average time of extinction and the standard deviation can be analytically calculated from the Master equation for a general decay process with a time-dependent rate of decay. The threshold for extinction is then the value of $x$ in Eq. (4.3) at time $t=\langle T\rangle$.

For instance, for the simple exponential decay process, the rate $k(t)=1$, and its integral $g(t)=t$ satisfy conditions $(4.2,4.6)$. Thus the average time of extinction for this process can be calculated from Eq. (4.8). This average time $\langle T\rangle$ can now be used to calculate the threshold of extinction, $\Theta$ in the solution for the continuous model, $\Theta\left(x_{0}\right)=x_{0} \exp (\langle T\rangle)$. In Figure 4.1, thresholds computed for various rates of decay and with various initial conditions are shown. It is interesting to note that the threshold value for different $k(t)$ are all less than one. 
Such stochastic processes can also be numerically simulated using the Gillespie algorithm (Gillespie, 1977). Starting from $t=0$ and $x=x_{0}$, in the case of the general decay process, the algorithm is used to generate the time at which the next particle decays based on the rate at which the reaction can occur. A uniform random number between 0 and $1, r$ is generated and the time of the next decay $t_{n}$ can be determined using, $r=\exp \left(-x \int_{t_{o}}^{t_{n}} k(t) d t\right)$ where $t_{o}$ is the time at which the previous decay had occurred. The averaged results from several Gillespie simulations are also shown in Fig. 4.1 and coincide with the predictions from the Master equation.

We observe that in units of population $[X]$, the threshold is a fraction (around 0.5) of a particle and the stronger the killing rate, the higher the threshold. For instance, in Figure 4.1, the threshold for the strong exponential rate of killing is much higher than that for the weak killing rate $1 /(t+\epsilon)$. Also, the threshold of extinction for oscillating rates is closer to $k(t)=1$, the higher the frequency of the oscillation.

\subsection{THRESHOLD FOR THE SIMPLE DECAY PROCESS FROM THE FOKKER-PLANCK AND LANGEVIN APPROXIMATIONS}

\subsubsection{Fokker-Planck Equation}

In the case of the decay process, the Master equation is completely solvable. However, in general, it is intractable and approximations are necessary to solve it. Fokker-Planck equations are obtained by approximating the "jumps" in a Master equation to be infinitely small (van Kampen, 2003). Results from a Fokker-Planck (F-P) approximation of the Master equation are often used to obtain the statistics of a stochastic process (Kepler \& Elston, 2001).

In the case of the simple decay process, the F-P equation can be derived from the Master equation by a Taylor expansion up to the second order that considers $x$ to be a continuous variable. Consider the simple exponential decay process where the decay rate $k(t)=1$. Then, the F-P equation for the probability density of $x$ varying in $t$ is 


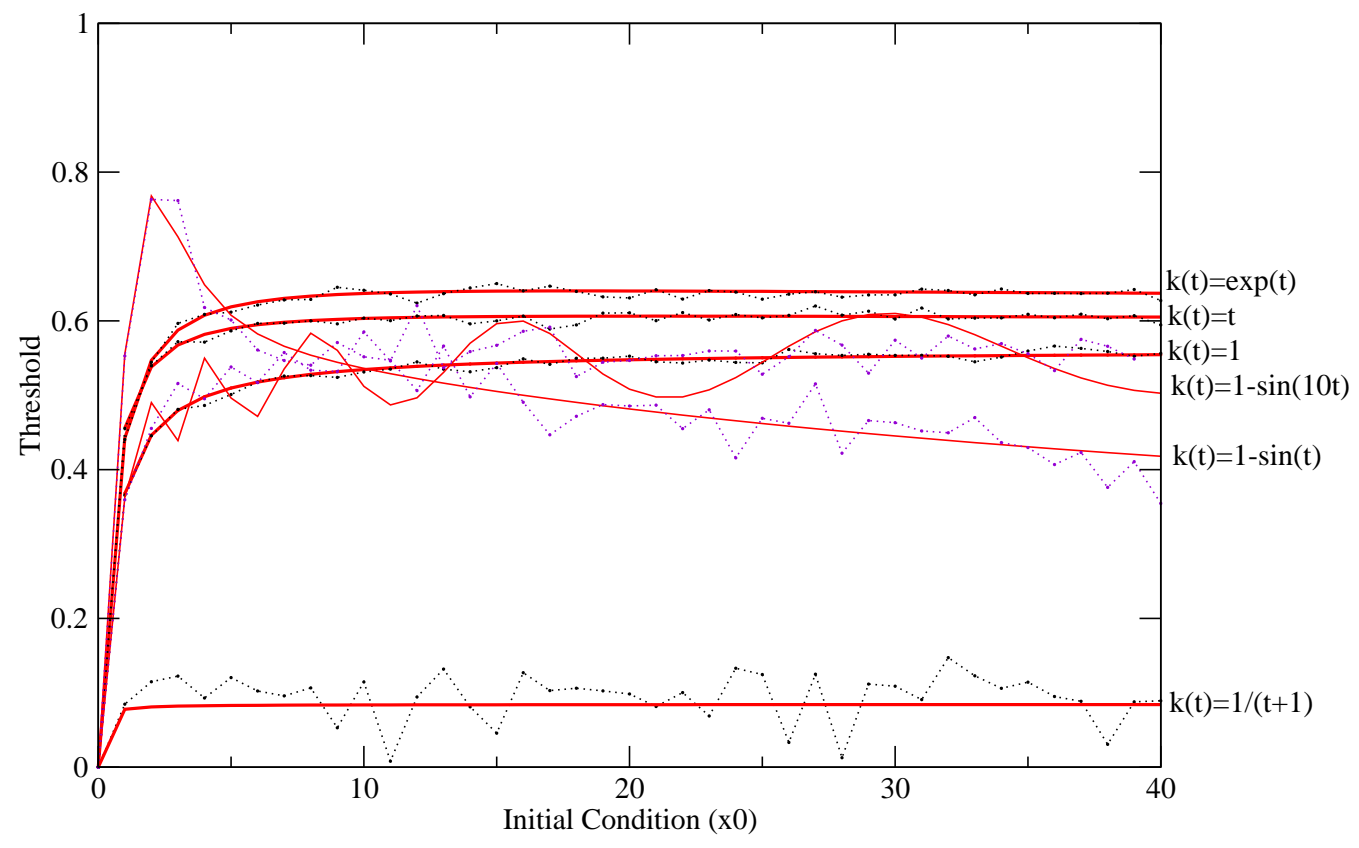

Figure 4.1: Threshold of extinction for various rates of decay - as predicted by the Master equation and Gillespie simulations. The rates $k(t)$ are marked on the right. The Gillespie simulations are averages of 10000 runs. (Gillespie results are shown as dotted lines. For the sinusoidal rates $k(t)=1-\sin (t)$ and $k(t)=1-\sin (10 t)$, the average of 1000 Gillespie runs is shown.) 


$$
\frac{\partial P\left(x, t \mid x_{0}, 0\right)}{\partial t}=\frac{\partial}{\partial x}\left[x P\left(x, t \mid x_{0}, 0\right)\right]+\frac{1}{2} \frac{\partial^{2}}{\partial x^{2}}\left[x P\left(x, t \mid x_{0}, 0\right)\right] .
$$

The goal again is to obtain the average time of extinction for a species obeying the above equation and compare it to that obtained from the Master equation. In particular, the problem can be thought of as a first passage time problem. First passage describes how long a particle whose position $x$ is described by the F-P remains in a region $L$. In this problem, the question posed is, what is the time $T$ it takes for a given experiment to decay to 0 particles and what is the average time $\langle T\rangle$ of several such experiments? Such a mean first passage time describes the average time to extinction.

The problem can be defined thus. Consider a population obeying the F-P equation (4.10), which has an absorbing boundary at $x=0$. At $x=b, b \gg 0$, consider another absorbing boundary. In this problem, if the initial condition is $P(x, 0)=\delta\left(x-x_{0}\right)$ where $x_{0}<b$, there is no probability for the population to increase and increase past $x_{0}$ to $x_{0}=b$. The only way to leave the region is to exit at $x=0$. (In Appendix 4.4.2, the same results are derived even if $x_{0}=b$ is assumed to be a reflecting boundary as long as $b \gg 0$.) Under these conditions, the probability at time $t$ that the population is still in the interval $(0, b)$ is $\int_{0}^{b} d x P\left(x, t \mid x_{0}, 0\right) \equiv G\left(x_{0}, t\right)$

$G\left(x_{0}, t\right)$ can also be thought of as the probability that the population leaves $(0, b)$ at a time $\langle T\rangle \geq t$ i.e., $G\left(x_{0}, t\right)=\int_{0}^{b} d x^{\prime} P\left(x, t \mid x_{0}, 0\right)=\operatorname{Prob}(\langle T\rangle \geq t)$. Then the mean first passage time out of the region $(0, b)$ is given by $\left\langle T\left(x_{0}\right)\right\rangle=-\int_{0}^{\infty} t \partial_{t} G\left(x_{0}, t\right) d t=\int_{0}^{\infty} G\left(x_{0}, t\right) d t$. It is possible to obtain an equation obeyed by $G\left(x_{0}, t\right)$ and hence $\left\langle T\left(x_{0}\right)\right\rangle$ from the backward F-P equation (with boundary condition $\left.P\left(x, 0 \mid x_{0}, 0\right)=\delta\left(x-x_{0}\right)\right)$ rather than from the Eq. (4.10) (Gardiner, 1985). Such an equation for $\left\langle T\left(x_{0}\right)\right\rangle$ can be solved with the appropriate boundary conditions (in this case $\langle T(0)\rangle=\langle T(b)\rangle=0$ (Appendix 4.4.2)) and an expression can be obtained for the mean time for a population decaying at the rate $k(t)=1$ to reach $x=0$. This is given by

$$
\left\langle T\left(x_{0}\right)\right\rangle=-2 \exp \left(2 x_{0}\right) I\left(x_{0}, b\right)-2 I(0, b)
$$

where $I(p, q)$ is defined as $\int_{p}^{q} \exp \left(-2 x^{\prime}\right) \ln \left(x^{\prime}\right) d x^{\prime}$. 
The above expression for $\left\langle T\left(x_{0}\right)\right\rangle$, Eq. (4.11) can be computed numerically for various initial conditions $x_{0}$ (by assuming $b$ to be a very large number). Results from such a calculation are shown in Figure 4.2 and are compared with the thresholds obtained from considering the full stochastic process using the Master equation. The threshold from the F-P approximation is much higher than that of the Master equation. This indicates that the F-P description is not a good approximation to determine extinction times.

\subsubsection{Langevin Equation}

Another simplification in capturing stochastic behavior is the Langevin approach. Here, the macroscopic behavior of the process is assumed to be known and stochastic effects are introduced by adding a 'noise source' to the equation of motion (under the restriction that the stationary solution reproduces the correct mean square fluctuations known from the dynamics of the problem) (van Kampen, 2003). Though, in general, the Langevin equation has to be derived systematically from the F-P equation, for the simple decay process the equation is given by,

$$
d x / d t=-x+\sqrt{x} \mathcal{L}(t)
$$

where the properties of $\mathcal{L}(t)$ are: its mean $\int \mathcal{L}\left(t^{\prime}\right) d t^{\prime}=0$ and its auto-correlation function $\int_{-\infty}^{\infty} \mathcal{L}(t) \mathcal{L}(t+\tau) d \tau=\delta(\tau)$. It can be proved that the statistics represented by Eq. (4.12) are the same as those of Eq. (4.10) when the Itô rule is used to interpret Eq. (4.12) (Page. 230-236 (van Kampen, 2003)).

The stochastic differential equation (4.12), can be evolved numerically where $\mathcal{L}(t)$ is a implemented by generating a random number with a Gaussian distribution (mean=0, variance=1). The average time to extinction can thus be obtained from the Langevin equation and can be used to estimate a threshold as well. These results are also plotted in Figure 4.2. Thus converting the deterministic ODEs to Langevin equations by adding noise terms is also not an accurate method to model stochastic processes.

Though the Langevin and F-P approximations are routinely used to obtain the statistics of a stochastic process, they should be used cautiously, as these examples demonstrate. 


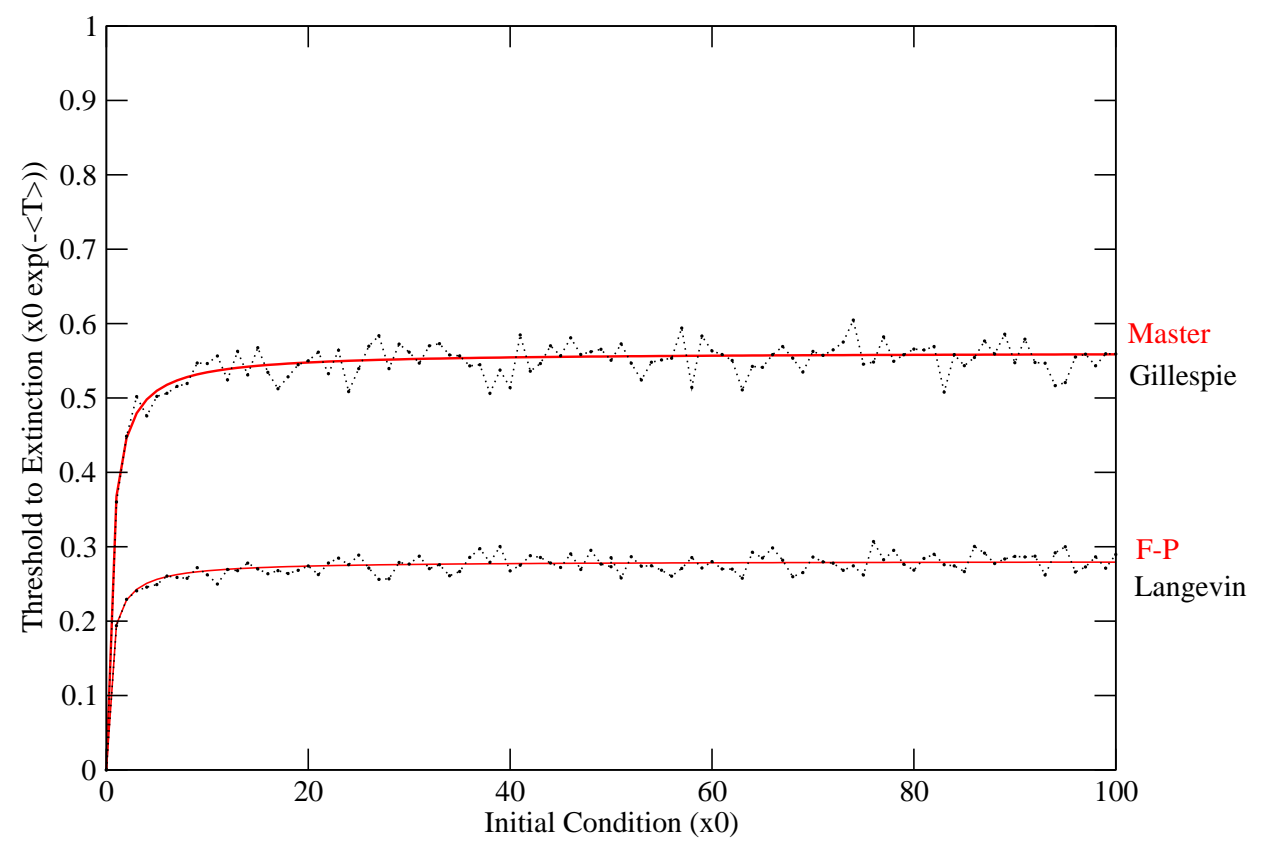

Figure 4.2: Time of extinction for decay process with rate $\mathrm{k}(\mathrm{t})=1$ using the various methods. (Results from Gillespie and Langevin methods are shown as dotted lines.) 


\subsection{THRESHOLD OF EXTINCTION FOR LARGER MODELS}

The aim of the above analysis was to predict an estimate for the threshold of extinction for deterministic population models, below which the population may be considered to be extinct. Can the threshold predicted by the general decay process be used as a "cut-off" for a general population model?

We tested our predictions by implementing the numerical Gillespie simulation of the modification of the 2 dimensional model (represented by the ODE system $(2.1,2.3)$ in Chapter 2) that captures pathogen- immune-cell dynamics. The reactions which constitute the model are as follows:

$$
\begin{array}{rll}
p & \stackrel{k_{1}}{\rightarrow} & 2 p \\
p+m & \stackrel{k_{2}}{\rightarrow} & 2 m \\
m & \stackrel{k_{3}}{\rightarrow} & \emptyset \\
m+m & \stackrel{k_{4}}{\rightarrow} & 3 m
\end{array}
$$

This is a stochastic simulation that models the fate of individuals in a population - hence $p$ and $m$ are measured as numbers of cells with dimensions, say $[X]$ (pathogens or immunecells). The first reaction (4.13) describes a pathogen dividing into 2 at the rate $k_{1}$. $k_{1}$ has the units of inverse time $1 /[T]$. Reaction (4.14) describes the interaction between a pathogen and an immune-cell. At the rate $k_{2}$, when a $p$ meets an $m$, the $p$ dies and a new $m$ is created. The rate $k_{2}$ has dimensions $1 /([T][X])$. Reaction (4.15) describes an immune-cell decaying at the rate $k_{3}$ where the dimensions of $k_{3}$ are also $1 /[T]$. Reaction (4.16) represents the positive feed-back of immune-cells at the rate $k_{4}$ where $k_{4}$ has units $1 /([T][X])$.

In implementing a stochastic simulation of the above interactions, detailed balance is implicitly assumed. (Technically detailed balance is defined as the condition that in stationary state the probability of going from state $n$ to $n^{\prime}$ is the same as the probability of the opposite transition from $n^{\prime}$ to $n$ i.e. $p(n) W\left(n, n^{\prime}\right)=p\left(n^{\prime}\right) W\left(n^{\prime}, n\right)$, where $p(x)$ is the probability to be in state $x$ and $W\left(x, x^{\prime}\right)$ is the transition probability from $x$ to $x^{\prime}$ (van Kampen, 2003). This is not necessarily the case in an ODE system). 
Another consideration while implementing a stochastic simulation is that outcomes that are not expected deterministically can also occur. For instance, in the above simulation, the pathogen is expected to grow, followed by growth in immune-cells. But chance fluctuations could result in the initial population of immune-cells to decay and the pathogen to grow at an exponential rate. Thus, although a deterministic model of the system predicts oscillations, in stochastic simulations, the pathogen and immune-cells go through a growth-death cycle at the end of which there is a high probability that the pathogen becomes extinct and the immune-cells also decay away.

A Gillespie algorithm is set up to simulate (4.13-4.16) (Gillespie, 1977). A random number is generated to determine the time of the next interaction and another random number is generated to determine which of the interactions occurs (based on the rate of each reaction). For the 2 random numbers $r_{1}$ and $r_{2}$, the time at which the next reaction occurs is,

$$
t_{n}=t_{o}+\left(1 / k_{0}\right) \ln \left(1 / r_{1}\right)
$$

where $t_{o}$ is the time at which the previous interaction has occurred and $k_{0}$ is the sum of the (time-independent) rates of the 4 reactions, $k_{0}=\sum_{\mu=1}^{4} k_{\mu}$. The interaction to be simulated is the $\mu^{t h}$ interaction for which $\sum_{j=1}^{\mu-1} k_{j}<r_{2} k_{0} \leq \sum_{j=1}^{\mu} k_{j}$.

The simulations are carried out for various initial conditions and parameters $\left(k_{1}, k_{2}, k_{3}, k_{4}\right)$ and the times to extinction are recorded. Now, the question arises, how is this time translated into a threshold for an ODE model? In the case of exponential decay, the predicted time of extinction was simply plugged into the solution for the ODE and a threshold was obtained. This should be done more carefully in the case of a more complicated model. The reactions (4.13-4.16) can be modeled using the following equations.

$$
\begin{aligned}
\frac{d p}{d t} & =k_{1} p-k_{2} m p \\
\frac{d m}{d t} & =k_{2} m p-k_{3} m+k_{4} m^{2},
\end{aligned}
$$

In general an ODE models the concentrations of the populations in question and it can be scaled to different units. Thus, $p$ and $m$ have dimensions of particle/volume or $[X] /[V]$. 
The parameters $k_{1}$ and $k_{3}$ still have dimensions $1 /[T]$ and can be identified exactly with the corresponding parameters in the discrete simulation (as long as the time scales are the same). Parameters $k_{2}$ and $k_{4}$ however reflect the scale as they have dimensions $[V] /([T][X])$ and have different numerical values depending on the scale at which the ODE model is chosen. (Note that $k_{2}$ is the rate at which the pathogens are killed and also the rate at which the immune-cells are created as a consequence of detailed balance.)

So to connect the stochastic and the deterministic model, a volume scale $[V]$ is chosen, say $V=1 \mathrm{ml}$. The rate constants and initial conditions are scaled accordingly and supplied to the Gillespie simulation, say $p(0)=p_{0}, m(0)=m_{0}$. Now, if a different scale were to be chosen for the same ODE model, say $V=2 \mathrm{ml}$, representing the same underlying biology (same growth rates, initial concentrations, interactions etc.), then the scaled initial conditions and parameters are supplied to the Gillespie algorithm (in this case, at double the volume and the same concentration, the initial conditions are $p(0)=2 p_{0}, m(0)=2 m_{0}$ and $k_{2}$ and $k_{4}$ are half that of the previous experiment.) As can be expected, stochastic simulations performed in larger volumes more closely mimic continuous models. (The above argument is not restricted even if the ODE does not model concentration but scales the population in any other general dimension. $[V]$ could be an area, length or even non-dimensional).

Keeping the above considerations in mind, a threshold is calculated by plugging the extinction times predicted by the Gillespie algorithm into the ODE. These thresholds are then compared to those predicted from the general one dimensional decay processes (Figure 4.3). We observe that they tend to lie within the range of error of thresholds predicted by the Master equation for a general decay process with decay rate $k(t)=\exp (t)$ in Section 4.1. (The error from the Master equation is in the time of extinction and is expanded to first order to find the error in the threshold.)

It is not entirely surprising that the thresholds of extinction for $(4.17,4.18)$ tend to the extinction threshold for $k(t)=\exp (t)$, as the rate at which $p$ is killed is $k_{2} m p$ and $m$ is growing at an exponential rate when $p$ is present. Thus a prediction from the Master equation of the threshold of extinction of a general decay process is a good estimate to determine when the population in a larger dimensional continuous model should be considered extinct.

The distribution of the thresholds of extinction, $\mathcal{F}(\Theta)$ from the Master equation for a 
given rate of decay $(k(t))$ can be specified by the mean and standard deviation as in Fig. 4.3. An algorithm can be devised, where the threshold is a random number $\theta$, generated with a (Gaussian) distribution $\mathcal{F}(\Theta)$, for every time-step of an ODE evolution. If the population value of the ODE is below $\theta$, the evolution of the ODE is stopped and if not, the next time step is computed. Such an algorithm could in principle capture extinction and even oscillations that may occur stochastically.

In many respects, the conclusions of this analysis are fairly intuitive. Deterministic models capture average behaviors of the actual stochastic process. We predict the time at which more than half of the possible experiments would have become extinct from a stochastic model and use that to cut off the deterministic simulation. Instead of choosing arbitrarily low thresholds to cut-off simulations as is often done, if the scales in the ODE are understood, simulations may be safely stopped when the population numbers are approximately $1 / 2$ in the scales chosen. 


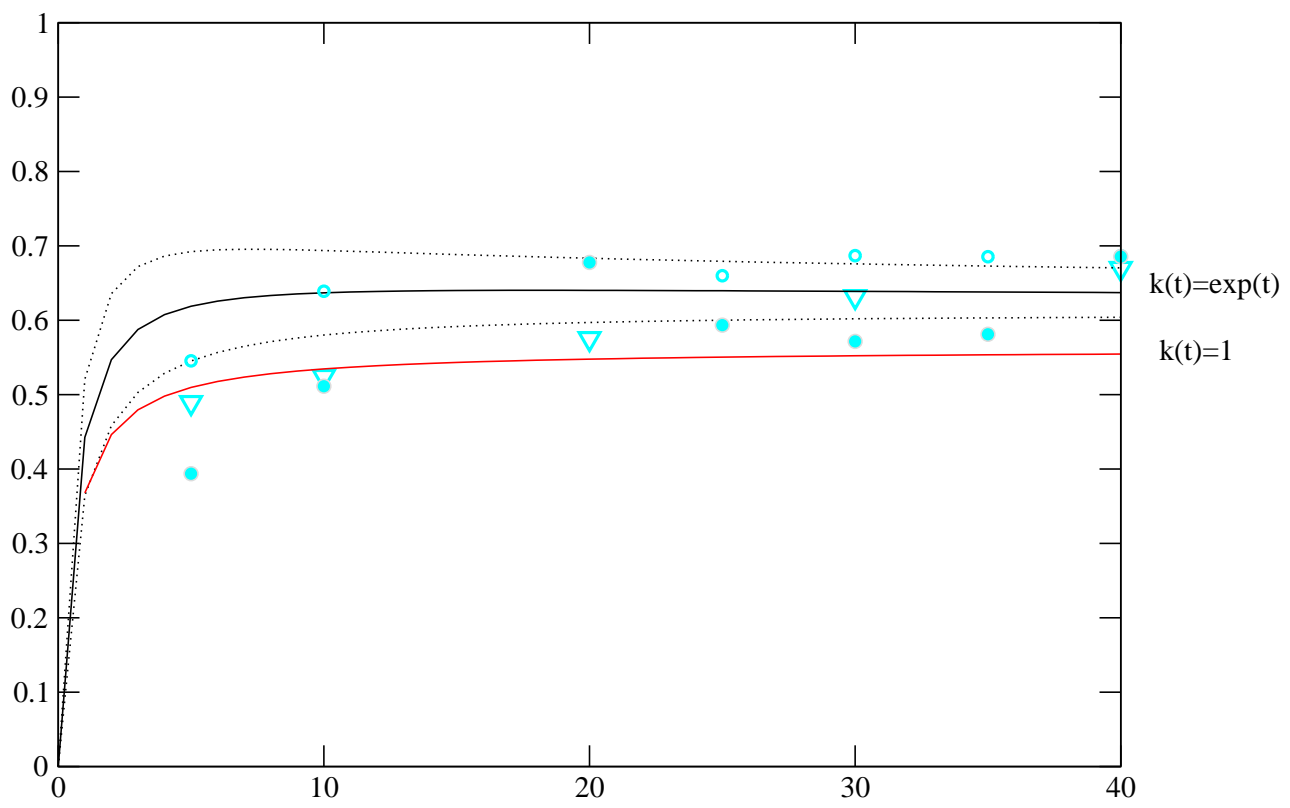

Figure 4.3: Threshold from Gillespie simulation of 2-d models with varying parameters compared with predicted values (for $k(t)=1$ and $k(t)=\exp (t)$ ) from the Master equation. The inverted triangles represent the parameter set $k_{1}=0.5, k_{2}=1.0, k_{3}=0.1$ and $k_{4}=0.01$. The circles represent the parameter set where there is no feed-back to $m$ i.e. $k_{4}=0$. The other parameters are $k_{1}=1.0, k_{3}=0.1$ and $k_{2}$ is 1.0 (filled) and 2.0 (unfilled). The scale for all simulations: ODE is $1 / 500$ times that of the Gillespie. 


\subsection{APPENDIX}

\subsection{1 $\langle T\rangle$ from the Master Equation}

The Master equation for a general decay process is given by Eq. 4.4,

$$
\frac{d P(x, t)}{d t}=k(t)[(x+1) P(x+1, t)-x P(x, t)]
$$

This equation needs to be solved with the following boundary condition, $P(x, 0)=\delta_{x, x_{0}}$ and $P\left(x_{0}+1, t\right)=0$. From Eq. (4.19), $P\left(x_{0}, t\right)$ can be obtained as follows,

$$
\frac{d P\left(x_{0}, t\right)}{d t}=-k(t) x_{0} P\left(x_{0}, t\right) \Rightarrow P\left(x_{0}, t\right)=\exp \left(-x_{0}\left(g(t)-g_{0}\right)\right)
$$

where $g(t)=\int k\left(t^{\prime}\right) d t^{\prime}$. Now, from (4.19), the equation obeyed by $P\left(x_{0}-1, t\right)$ is as follows:

$$
\frac{d P\left(x_{0}-1, t\right)}{d t}=k(t)\left[x_{0} P\left(x_{0}, t\right)-\left(x_{0}-1\right) P\left(x_{0}-1\right)\right.
$$

with solution

$$
P\left(x_{0}-1, t\right)=x_{0} \exp \left(\left(x_{0}-1\right)\left(g(t)-g_{0}\right)\right)\left[1-\exp \left(g(t)-g_{0}\right)\right]
$$

We can prove by induction that the solution of $P(n, t)$ is

$$
P(n, t)=\left(\begin{array}{c}
x_{0} \\
n
\end{array}\right)\left[\exp \left(-\left(g(t)-g_{0}\right)\right)\right]^{n}\left[1-\exp \left(-\left(g(t)-g_{0}\right)\right]^{x_{0}-n}\right.
$$

and show that the solution for $P(n-1, t)$ has the same form as $P(n, t)$. The equation obeyed by $P(n-1, t)$ if Eq. (4.20) is true, is

$$
\begin{aligned}
& \frac{d P(n-1, t)}{d t}= \\
& \quad k(t)\left[\left(n\left(\begin{array}{c}
x_{0} \\
n
\end{array}\right)\left[\exp \left(-\left(g(t)-g_{0}\right)\right)\right]^{n}\left[1-\exp \left(-\left(g(t)-g_{0}\right)\right)\right]^{x_{0}-n}\right)-((n-1) P(n-1, t))\right] .
\end{aligned}
$$


Moving the $P(n-1, t)$ term to the left hand side, and multiplying both sides by the integrating factor $\exp (g(t)(n-1))$ and combining, we get

$$
\begin{aligned}
& \frac{d(P(n-1, t)(\exp (g(t)(n-1))))}{d t}= \\
& k(t)\left(n\left(\begin{array}{c}
x_{0} \\
n
\end{array}\right)\left[\exp \left(-\left(g(t)-g_{0}\right)\right)\right]^{n}\left[1-\exp \left(-\left(g(t)-g_{0}\right)\right)\right]^{x_{0}-n}\right) \exp (g(t)(n-1)) .
\end{aligned}
$$

Now, integrate both sides with respect to $t$. The right hand side can be integrated by parts by assuming, say $y=1-\exp \left(g(t)-g_{0}\right)$, so the equation reduces to

$$
P(n-1, t) \exp \left((g(t)(n-1))=n\left(\begin{array}{c}
x_{0} \\
n
\end{array}\right) \exp \left((n-1) g_{0}\right) \int y^{x_{0}-n} d y\right.
$$

Integrating and re-arranging, we get,

$$
\begin{aligned}
& P(n-1, t)= \\
& \quad \frac{n}{x_{0}-n+1}\left(\begin{array}{c}
x_{0} \\
n
\end{array}\right) \exp \left((n-1) g_{0}\right) \exp (-(g(t)(n-1)))\left[1-\exp \left(-\left(g(t)-g_{0}\right)\right)\right]^{x_{0}-n+1} \\
& \quad+C \exp (-g(t)(n-1)),
\end{aligned}
$$

where $\mathrm{C}$ is the constant from integration. Now, applying the boundary condition that $P(n-1,0)=0$ (because at $\left.t=0, P(x, 0)=\delta\left(x, x_{0}\right)\right) \Rightarrow C=0$.

Simplifying, we get,

$$
P(n-1, t)=\left(\begin{array}{c}
x_{0} \\
(n-1)
\end{array}\right)\left[\exp \left(-\left(g(t)-g_{0}\right)\right)\right]^{n-1}\left[1-\exp \left(-\left(g(t)-g_{0}\right)\right)\right]^{x_{0}-n-1}
$$

which is the same as the form for the solution of $P(n, t)$, Eq. (4.20).

Now, that the solution for the Master equation is known, we proceed to find the average time of extinction. The probability distribution that the population goes to 0 in the time interval between $t$ and $t+d t, \Pi(t)$ is given by

$$
\begin{aligned}
& \Pi(t)=\frac{d P(0, t)}{d t}= \\
& \quad x_{0} k(t)\left[1-\exp \left(-\left(g(t)-g_{0}\right)\right)\right]^{x_{0}-1} \exp \left(-\left(g(t)-g_{0}\right)\right) .
\end{aligned}
$$


The average time to extinction is then given by $\int_{0}^{\infty} t^{\prime} \Pi\left(t^{\prime}\right) d t^{\prime}$ under the condition that $\int_{0}^{\infty} \Pi\left(t^{\prime}\right) d t^{\prime} \equiv 1$.

The normalization condition for $\Pi(t)$ can be proved thus.

$$
\int_{0}^{\infty} \Pi\left(t^{\prime}\right) d t^{\prime}=\int_{0}^{\infty} k\left(t^{\prime}\right)\left[1-\exp \left(-\left(g\left(t^{\prime}\right)-g_{0}\right)\right)\right]^{x_{0}-1} \exp \left(-\left(g\left(t^{\prime}\right)-g_{0}\right)\right) .
$$

Set $y=1-\exp \left(-g\left(t^{\prime}\right)-g_{0}\right)$, then,

$$
\begin{aligned}
\int_{0}^{\infty} \Pi\left(t^{\prime}\right) d t^{\prime} & =x_{0} \int_{0}^{1} y^{x_{0}-1} d y \\
& =1
\end{aligned}
$$

The limits can be set as above and the function normalized only under the following condition: $g(\infty)=\infty$ as specified by Eq. (4.6).

Next, we derive an expression for average $T,\langle T\rangle$ where

$$
\langle T\rangle=\int_{0}^{\infty} t^{\prime} \Pi\left(t^{\prime}\right) d t^{\prime}
$$

Substituting the expression for $\Pi(t)$, we get,

$$
\langle T\rangle=x_{0} \int_{0}^{\infty} t^{\prime} x_{0} k\left(t^{\prime}\right)\left[1-\exp \left(-\left(g\left(t^{\prime}\right)-g_{0}\right)\right)\right]^{x_{0}-1} \exp \left(-\left(g\left(t^{\prime}\right)-g_{0}\right)\right) .
$$

Expanding $\left[1-\exp \left(-\left(g\left(t^{\prime}\right)-g_{0}\right)\right)\right]^{x_{0}-1}$ as a binomial series and taking the summation sign out of the integration sign,

$$
\langle T\rangle=x_{0} \sum_{j=0}^{x_{0}-1}-1^{j}\left(\begin{array}{c}
x_{0}-1 \\
j
\end{array}\right) \int_{0}^{\infty} t^{\prime} k\left(t^{\prime}\right) \exp \left(-g\left(t^{\prime}\right)-g_{0}\right) d t^{\prime}
$$

Integrating by parts by setting $t^{\prime}=u$ and $k\left(t^{\prime}\right) \exp \left(-(j+1)\left(g\left(t^{\prime}\right)-g_{0}\right)\right) d t^{\prime}=d v$, and substituting the condition (4.6),

$$
\langle T\rangle=x_{0} \sum_{j=0}^{x_{0}-1} \frac{-1^{j}}{j+1}\left(\begin{array}{c}
x_{0}-1 \\
j
\end{array}\right) \int_{0}^{\infty} \exp \left(-(j+1)\left(g\left(t^{\prime}\right)-g_{0}\right)\right) d t^{\prime} .
$$

Similarly, integrating by parts twice, we get,

$$
\left\langle T^{2}\right\rangle=2 x_{0} \sum_{j=0}^{x_{0}-1} \frac{(-1)^{j}}{j+1}\left(\begin{array}{c}
x_{0}-1 \\
j
\end{array}\right) \int_{0}^{\infty} t^{\prime} \exp \left(-(j+1)\left(g\left(t^{\prime}\right)-g_{0}\right)\right) d t^{\prime} .
$$




\subsection{2 $\langle T\rangle$ from the Fokker-Planck Equation}

Calculating the average time of extinction from the F-P equation closely follows the method explained in Page.136-138 of (Gardiner, 1985). The F-P equation obeyed by a population of decaying particles is given by Equation 4.10,

$$
\frac{\partial P\left(x, t \mid x_{0}, 0\right)}{\partial t}=\frac{\partial}{\partial x}\left[x P\left(x, t \mid x_{0}, 0\right)\right]+\frac{1}{2} \frac{\partial^{2}}{\partial x^{2}}\left[x P\left(x, t \mid x_{0}, 0\right)\right] .
$$

Let the initial population at time $t=0$ be $x=x_{0}$. The question is how long does the population remain in the interval $(0, b)$ which contains $x$, such that $0 \leq x_{0} \ll b$. We assume that 0 and $b$ are absorbing boundaries (for now). Under these conditions, the probability that at time $t$, the particle is still in $(0, b)$ is given by $\int_{0}^{b} d x P\left(x, t \mid x_{0}, 0\right) \equiv G\left(x_{0}, t\right)$ (i.e. starting at $x=x_{0}$ at $t=0$, integrate over the probability of being at any $x$ such that $\left.0 \leq x \ll b\right)$. $G\left(x_{0}, t\right)$ can also be thought of as probability that the population leaves $(0, b)$ at a time $T \geq t$ i.e., $G\left(x_{0}, t\right)=\int_{0}^{b} d x P(x, t \mid x, 0)=\operatorname{Prob}(T \geq t)$. Since the system is homogeneous in time, the backward F-P equation can be written using the same boundary (initial) condition $P\left(x, t \mid x_{0}, 0\right)=P\left(x, 0 \mid x_{0},-t\right)$ so that,

$$
\frac{\partial P\left(x, t \mid x_{0}, 0\right)}{\partial t}=x_{0} \frac{\partial P\left(x, t \mid x_{0}, 0\right)}{\partial x_{0}}+\frac{1}{2} x_{0} \frac{\partial^{2} P\left(x, t \mid x_{0}, 0\right)}{\partial x_{0}^{2}} .
$$

Integrating the backward F-P with respect to $x$ (which is not as trivial in the forward F-P), we get

$$
\frac{\partial G\left(x_{0}, t\right)}{\partial t}=x_{0} \frac{\partial G\left(x_{0}, t\right)}{\partial x_{0}}+\frac{1}{2} x_{0} \frac{\partial^{2} G\left(x_{0}, t\right)}{\partial x_{0}^{2}}
$$

obeyed by $G\left(x_{0}, t\right)$. Now, the boundary conditions are

$P\left(x, 0 \mid x_{0}, 0\right)=\delta\left(x-x_{0}\right)$

and hence,

$G\left(x_{0}, 0\right)=1$ when $0 \leq x_{0} \leq b$

$G\left(x_{0}, 0\right)=0$ elsewhere and

$G(0, t)=G(b, t)=0$.

Now, the mean first passage time out of the region $(0, b)$ is given by

$$
\left\langle T\left(x_{0}\right)\right\rangle=-\int_{0}^{\infty} t \partial_{t} G\left(x_{0}, t\right) d t=\int_{0}^{\infty} G\left(x_{0}, t\right) d t
$$


and obeys the following equation which is obtained by integrating 4.21 from 0 to $\infty$ with respect to $t$ :

$$
x_{0} \frac{\partial\left\langle T\left(x_{0}\right)\right\rangle}{\partial x_{0}}+\frac{1}{2} x_{0} \frac{\partial^{2}\left\langle T\left(x_{0}\right)\right\rangle}{\partial x_{0}^{2}}=-1 .
$$

(This result uses the fact that $G\left(x_{0}, \infty\right)=0$ and $G\left(x_{0}, 0\right)=1$.) Now for the absorbing boundaries, $T(0)=T(b)=0$.

Equation 4.22 can be re-arranged as

$$
\begin{gathered}
\frac{\partial^{2}\left\langle T\left(x_{0}\right)\right\rangle}{\partial x_{0}{ }^{2}}-2 \frac{\partial\left\langle T\left(x_{0}\right)\right\rangle}{\partial x_{0}}=-\frac{2}{x_{0}} . \\
\Rightarrow\left\langle T\left(x_{0}\right)\right\rangle=-D \exp \left(2 x_{0}\right)-2 \exp \left(2 x_{0}\right) I\left(0, x_{0}\right)+\frac{C}{2}\left(\exp \left(2 x_{0}-1\right)\right)
\end{gathered}
$$

where $C$ and $D$ are constants of integration and

$$
I(p, q) \equiv \int_{p}^{q} \exp \left(2 x^{\prime}\right) \ln \left(x^{\prime}\right) d x^{\prime}
$$

Applying boundary conditions,

$$
\begin{aligned}
\langle T(0)\rangle & =0 \Rightarrow D=0 \\
\langle T(b)\rangle & =0 \Rightarrow C=\frac{4 \exp (2 b) I(0, b)}{\exp (2 b)-1} \\
\Rightarrow\left\langle T\left(x_{0}\right)\right\rangle & =-2 \exp \left(2 x_{0}\right) I\left(0, x_{0}\right)+\frac{2 \exp (2 b) I(0, b)}{\exp (2 b)-1}\left(\exp \left(2 x_{0}-1\right)\right.
\end{aligned}
$$

Substituting $I(0, b)=I\left(0, x_{0}\right)+I\left(x_{0}, b\right)$ and $\lim _{b \rightarrow \infty} \frac{\exp (2 b)}{\exp (2 b)-1}=1$,

$$
\begin{aligned}
\left\langle T\left(x_{0}\right)\right\rangle & =-2 \exp \left(2 x_{0}\right)\left[I(0, b)-I\left(x_{0}, b\right)\right]+2 I(0, b)\left(\exp \left(2 x_{0}-1\right)\right) \\
\Rightarrow\left\langle T\left(x_{0}\right)\right\rangle & =2 \exp \left(2 x_{0}\right) I\left(x_{0}, b\right)-2 I(0, b)
\end{aligned}
$$

The above analysis assumes two absorbing boundaries where the boundary at $b$ is only an approximation because physically there is no absorbing boundary. Here, we prove that even 
if $b$ is assumed to be a reflecting boundary, the expression for $\langle T\rangle$ remains the same. Equation (4.22) is now solved with the boundary conditions $\left\langle T\left(x_{0}=0\right)\right\rangle=0$ and $\frac{\partial\left\langle T\left(x_{0}=b\right)\right\rangle}{\partial x_{0}}=0$.

$$
\begin{aligned}
\langle T(0)\rangle & =0 \Rightarrow D=0 \\
\frac{\partial\left\langle T\left(x_{0}=b\right)\right\rangle}{\partial x_{0}} & =0 \Rightarrow C=4 I(0, b)+2 \exp (-2 b) \ln (b) \\
\Rightarrow\left\langle T\left(x_{0}\right)\right\rangle & =-2 \exp \left(2 x_{0}\right) I\left(0, x_{0}\right)+(2 I(0, b)+(\exp (2 x)-1))
\end{aligned}
$$

Substituting $I(0, b)=I\left(0, x_{0}\right)+I\left(x_{0}, b\right)$ and $\lim _{b \rightarrow \infty} \exp (-2 b) \ln (b)=0$,

$$
\Rightarrow\left\langle T\left(x_{0}\right)\right\rangle=2 \exp \left(2 x_{0}\right) I\left(x_{0}, b\right)-2 I(0, b)
$$




\subsection{BIBLIOGRAPHY}

Abraham, E., Anzueto, A., Gutierrez, G., Tessler, S., San Pedro, G., Wunderink, R., Dal Nogare, A., Nasraway, S., Berman, S., Cooney, R., Levy, H., Baughman, R., Rumbak, M., Light, R., Poole, L., Allred, R., Constant, J., Pennington, J. \& Porter, S. (1998). Double-blind randomised controlled trial of monoclonal antibody to human tumour necrosis factor in treatment of septic shock. Lancet, 351(9107), 929-933. NORASEPT II Study Group.

Abramova, F., Grinberrg, L., Yampolskaya, O. \& Walker, D. (1993). Pathology of inhalational anthrax in 42 cases from the Sverdlovsk outbreak of 1979. Proc Natl Acad Sci. 90, 2291-2294.

Abramson, G. \& Zanette, D. (1998). Statistics of extinction and survival in Lotka-Volterra systems. Phy Rev. E, 57(4), 4572-4577.

ACCP/SCC (1992). American College of Chest Physicians/Society of Critical Care Medicine Consensus Conference: Definitions for sepsis and organ failure and guidelines for the use of innovative therapies in sepsis. Crit Care Med. 20, 864-874.

Afrajmovich, V., Arnol'd, V., Il'yashenko, Y. \& Shil'nikov, L. (1988). Dynamical Systems vol. 5, of Encyclopedia of Mathematical Sciences chapter 1. New York: Springer-Verlag.

Alberti, C., Brun-Buisson, C., Burchardi, H., Martin, C., Goodman, S., Artigas, A., Sicignano, A., Palazzo, M., Moreno, R., Boulme, R., Lepage, E. \& Le Gall, J. (2002). Epidemiology of sepsis and infection in ICU patients from an international multicentre cohort study. Int Care Med, , 28, 108-121.

An, G. (2001). Agent based computer simulation and SIRS: building a gap between basic science and clinical trials. Shock, , 16, 266-273. 
Angus, D., Linde-Zwirble, W., Lidicker, J., Clermont, G., Carcillo, J. \& Pinsky, M. (2001). Epidemiology of severe sepsis in the United States: analysis of incidence, outcome, and associated costs of care. Crit Care Med., 29(7), 1303-1310.

Antia, R., Koella, J. \& Perrot, V. (1996). Models of the within-host dynamics of persistent mycobacterial infections. Proc R Soc Lond B Biol Sci., 263(1368), 257-263.

Arrowsmith, D. \& Place, C. (1990). An introduction to dynamical systems, chapter 3. Cambridge: Cambridge University Press, 1 edition.

Babior, B. M. (2000). Phagocytes and oxidative stress. Am.J.Med., 109, 33-44.

Bartlett, M. (1966). An introduction to stochastic processes with special reference to methods and applications. Cambridge University Press, Cambridge.

Beatty, W., Morrison, R. \& Byrne, G. (1994). Persistent chlamydiae : from cell culture to a paradigm for chlamydial pathogenesis. Microbiol. Rev. 58, 686-699.

Bellingan, G. (1999). Inflammatory cell activation in sepsis. Br. Med. Bull. 55, 12-29.

Bernard, G., Vincent, J., Laterre, P., LaRosa, S., Dhainaut, J., Lopez-Rodriguez, A., Steingrub, J., Garber, G., Helterbrand, J., Ely, E. \& Fisher, C. J. (2001). Efficacy and safety of recombinant human activated protein C for severe sepsis. New Eng J Med, 344(10), 699-709.

Beutler, B. (2000). Endotoxin, toll-like receptor 4, and the afferent limb of innate immunity. Curr.Opin.Microbiol. 3, 23-28.

Bogdan, C. (2001). Nitric oxide and the immune response. Nat.Immunol. 2, 907-916.

Bogdan, C., Vodovotz, Y. \& Nathan, C. (1991). Macrophage deactivation by interleukin 10. J.Exp.Med. 174, 1549-155.

Bone, R. (1993). The search for a magic bullet to fight sepsis. JAMA, 269(17), 2221-2227.

Bone, R. (1996a). Immunologic Dissonance: A Continuing Evolution in Our Understanding of the Systemic Inflammatory Response Syndrome (SIRS) and the Multiple Organ Dysfunction Syndrome (MODS). Ann Intern Med, 125(8), 680-687.

Bone, R. (1996b). Towards a theory regarding the pathogenesis of the systemic inflammatory response syndrome: what we do and do not know about cytokine regulation. Crit Care Med, 24(1), 163-172. 
Bone, R., Balk, R., Cerra, F., Dellinger, R., Fein, A., Knaus, W., Schein, R. \& Sibbald, W. (1992). Definitions for sepsis and organ failure and guidelines for the use of innovative therapies in sepsis. Chest, 101(6), 1644-1655. The ACCP/SCCM Consensus Conference Committee. American College of Chest Physicians/Society of Critical Care Medicine.

Bonventre, J. (2003). Molecular response to cytotoxic injury: role of inflammation, MAP kinases, and endoplasmic riticulum stress response. Semin. Nephrol. 23(5), 439-448.

Brossier, F., Weber-Levy, M., Mock, M. \& Sirard, J. (2000). Role of toxin functional domains in anthrax pathogenesis. Infect Immun. 68(4), 1781-1786.

Buchman, T., Cobb, J., Lapedes, A. \& Kepler, T. (2001). Complex systems analysis : a tool for shock research. Shock, 16(4), 248-251.

Cavaillon, J. M. (1994). Cytokines and macrophages. Biomed.Pharmacother. 48, 445-453.

Chong, H., Vodovotz, Y., Cox, G. \& Barcellos-Hoff, M. (1999). Immunocytochemical detection of latent tgf- $\beta$ activation in cultured macrophages. J.Cell.Physiol. 178, 275-283.

Clermont, G., Bartels, J., Kumar, R., Constantine, G., Vodovotz, Y. \& Chow, C. (2004). In Silico design of clinical trials : A method coming of age. Crit Care Med, 21, 77-85.

Cross, A. \& Opal, S. (2003). A new paradigm for the treatment of sepsis: is it time to consider combination therapy? Ann Intern Med. 138(6), 502-505.

Dai, Z. \& Koehler, T. (1997). Regulation of anthrax toxin activator gene (atxA) expression in Bacillus Anthracis : temparature, not CO2/bicarbonate, affects AtxA synthesis. Infect Immun. 65, 2576-2582.

Day, J. Personal communication. Work in progress.

de Waal Malefyt, R., Abrams, J., Bennett, B., Figdor, C. \& De Vries, J. (1991). Interleukin 10 (il-10) inhibits cytokine synthesis by human monocytes: an autoregulatory role of il-10 produced by monocytes. J.Exp.Med. 174, 1209-1220.

Dixon, T., Meselson, M., Guillemin, J. \& Hanna, P. (2004). Anthrax. N Engl J Med, 341(11), 815-827.

Djeu, J. Y., Serbousek, D. \& Blanchard, D. (1990). Release of tumor necrosis factor by human polymorphonuclear leukocytes. Blod, 76, 1405-1409. 
Doering, C. \& Sander, L. (2004). Extinction times for birth-deat processes: continuum asymptotics, and the failure of the Fokker-Planck approximation. arXiv: q-bio, 0401016v1, 1-20.

Durrett, R. \& Levin, S. (1994). The importance of being discrete (and spatial). Theo Pop Biology, 46, 363-394.

Eiseman, B., Beart, R. \& Norton, L. (1977). Multiple organ failure. Surgery, Gynecology and Obstetrics, 144, 323-326.

Ermentrout, B. (2002). Simulating, Analyzing, and Animating Dynamical Systems. A Guide to XPPAUT for Researchers and Students. SIAM.

Fisher, C., Opal, S. \& Dhainaut, e. a. (1993). Influence of anti TNF monoclonal antibody on cytokine levels in patients with sepsis. The sepsis syndrome study group. Crit Care Med, 27, 749-755.

Florquin, S., Amraoui, Z., Dubois, C., Decuyper, J. \& Goldman, M. (1994). The protective role of endogenously synthesized nitric oxide in staphylococcal enterotoxin b-induced shock in mice. J.Exp.Med, 180, 1153-1158.

Freeman, B. D. \& Natanson, C. (2000). Anti-inflammatory therapies in sepsis and septic shock. Expert.Opin.Investig.Drugs, 9, 1651-1663.

Friedlander, A. (1986). Macrophages are sensitive to anthrax lethal toxin through an aciddependent process. J Biol Chem. 261(16), 7123-7126.

Friedlander, A., Welkos, S. \& Pitt, M. (1993). Postexposure prophylaxis against experimental inhalational anthrax. J Infect Dis. 167, 1239-1242.

Gandhi, A., Levin, S. \& Orzag, S. (1998). "Critical slowing down" in time-to-extinction : an example of critical phenomena in ecology. $J$ Theo Bio, 192, 363-376.

Garcia, R. \& Teixeira, M. (2004). Vector fields on manifolds with boundary and reversibility - an expository account. Qualitative theory of dynamical systems, 5, 185-201.

Gardiner, C. (1985). Handbook of stochastic methods. Springer-Verlag.

Gillespie, D. (1977). Exact stochastic simulation of coupled chemical reactions. J Phy Chem. 81, 2340-2361.

Hanna, P., Acosta, D. \& Collier, J. (1993). On the role of macrophages in anthrax. Proc Natl Acad Sci. 90, 10198-10201. 
Harbrecht, B. G., Billiar, T., Stadler, J., Demetris, A., Ochoa, J., Curran, R. \& Simmons, R. (1992). Nitric oxide synthesis serves to reduce hepatic damage during acute murine endotoxemia. Crit.Care Med. 20, 1568-1574.

Hotchkiss, R. \& Karl, R. (2003). The Pathophysiolgy and Treatment of Sepsis. N Engl J Med, 348(2), 138-150.

Jaeschke, H. \& Smith, C. (1997). Mechanisms of neutrophil-induced parenchymal cell injury. J Leukoc Biol. 61(6), 647-653.

Janeway, C. \& Travers, P. (1997). Immunobiology: The Immune System in Health and Disease (III Edn.). Current Biology Ltd., Garland.

Johnson, M. L. \& Billiar, T. (1998). Roles of nitric oxide in surgical infection and sepsis. World J.Surg. 22, 187-196.

Kalns, J., Morris, J., Eggers, J. \& Kiel, J. (2002). Delayed treatment with doxycyline has limited effect on anthrax infection in BLK57/B6 mice. Biochem Biophys Res Commun. 297(3), 506-509.

Kepler, T. \& Elston, T. (2001). Stochasticity in transcriptional regulation : origins, consequences and mathematical representations. Biophysical J, 81, 3116-3136.

Kirschner, D. \& Blaser, M. (1995). The dynamics of helicobacter pylori infection of the human stomach. J Theor Biol. 176, 281-290.

Kumar, R. \& Chow, C. The threshold of extinction of decaying populations in a deterministic model. In preparation.

Kumar, R., Chow, C., Clermont, G. \& Vodovotz, Y. (2001). A theoretical model for the simulation of sepsis. J Leuk Biol. Suppl:90, 10-20.

Kumar, R., Chow, C., Clermont, G. \& Vodovotz, Y. Mathematical simulation of anthrax infection. Submitted to Infection and Immunity.

Kumar, R., Clermont, G., Vodovotz, Y. \& Chow, C. (2004b). Dynamics of acute inflammation. J Theor Biol. 230(2), 145-155.

Kuznetsov, Y. (1998). Elements of applied bifurcation theory (Applied Mathematical Sciences, Vol.112). Springer-Verlag.

Levy, M., Fink, M., Marshall, J., Abraham, E., Angus, D., Cook, D., Cohen, J., Opal, S., Vincent, J. \& Ramsay, G. (2003). International sepsis definitions conference. Intensive 
Care Med. 29(4), 530-538. 2001 SCCM/ESICM/ACCP/ATS/SIS International Sepsis Definitions Conference.

Louis, E., Franchimont, A. \& Piron, A., e. a. (1998). TNF gene polymorphism influences TNF-alpha production in LPS stimulated whole blood culture in healthy humans. FIND OUT, 113, 401-406.

Luckhart, S., Crampton, A., Zamora, R., Lieber, M., Dos Santos, P., Peterson, T., Emmith, N., Lim, J., Wink, D. \& Vodovotz, Y. (2003). Mammalian transforming growth factor$\beta 1$ activated after ingestion by Anopheles stephensi modulates mosquito immunity. Infect.Immun. 71, 3000-3009.

Maitra, S. K., Rachmilewitz, D., Eberle, D. \& Kaplowitz, N. (1981). The hepatocellular uptake and biliary excretion of endotoxin in the rat. Hepatology, 1, 401-407.

Marshall, J., Vincent, J., Fink, M., Cook, D., Rubenfeld, G., Foster, D., Fisher, C. J., Faist, E. \& K, R. (2003). Measures, markers, and mediators: toward a staging system for clinical sepsis. A report of the Fifth Toronto Sepsis Roundtable, Toronto, Ontario, Canada, October 25-26, 2000. Crit Care Med. 31, 1560-1567.

McCloskey, R., Straube, R., Sanders, C., Smith, S. \& Smith, C. (1994). Treatment of septic shock with human monoclonal antibody HA-1A. A randomized, double-blind, placebocontrolled trial. Ann Intern Med. 121(1), 1-5. CHESS Trial Study Group.

Moayeri, M., Haines, D., Young, H. \& Leppla, S. (2003). bacillus anthracis lethal toxin induces tnf- $\alpha$-independent hypoxia-mediated toxicity in mice. J Clin Inv. 112(5), 670682.

Muckart, D. \& Bhagwanjee, S. (1997). American College of Chest Physicians/Society of Critical Care Medicine Consensus Conference definitions of the systemic inflammatory response syndrome and allied disorders in relation to critically injured patients. Crit Care Med. 25, 1789-1795.

Nasell, I. (1995). Epidemic models : their structure and relation to data. Cambridge University Press, New-York.

Nasell, I. (1999). On the time to extinction in recurrent epidemics. J Roy Stat Soc B, 61(2), 309-330. 
Nasell, I. (2001). Extinction and quasi-stationarity in the Verhulst logistic model. J Theo Bio, 211, 11-27.

Nathan, C. F. \& Hibbs, J. J. (1991). Role of nitric oxide synthesis in macrophage antimicrobial activity. Curr.Opin.Immunol. 3, 65-70.

O’Brien, J., Friedlander, A., Dreier, T., Ezzell, J. \& Leppla, S. (1985). Effect of anthrax toxin components on human neutrophils. Infect Immun. 47, 306-310.

Opal, S. M. \& DePalo, V. (2000). Anti-inflammatory cytokines. Chest, 117, 1162-1167.

Park, J., Chang, S., Lee, K. \& Shin, S. (1996). Protective effect of nitric oxide in an endotoxin-induced septic shock. Am.J.Surg. 171, 340-345.

Parker, S. J. \& Watkins, P. (2001). Experimental models of gram-negative sepsis. Br J Surg, $88,22-30$.

Peixoto, M. (1962). Structural stability on two dimensional manifolds. Topology, 1, 101-120.

Pezard, C., Weber, M., Sirard, J., Berche, P. \& Mock, M. (1995). Protective immunity induced by Bacillus Anthracis toxin-deficient strains. Infect Immun. 63(4), 1369-1372.

Pinsky, M. R. (2001). Sepsis: a pro- and anti-inflammatory disequilibrium syndrome. Contrib.Nephrol. 300, 354-366.

Polidori, D. \& Trimmer, J. (2003). Bringing advanced therapies to marker faster: a role for bio-simulation? Diabetes' Voice, 48(2), 28-30.

Prince, A. (2003). The host response to anthrax lethal toxin: unexpected observations. $J$ Clin Inv. 112(5), 656-658.

Rees, D. D., Cunha, F., Assreuy, J., Herman, A. \& Moncada, S. (1995). Sequential induction of nitric oxide synthase by corynebacterium parvum in different organs of the mouse. Br.J.Pharmacol. 114, 689-693.

Reinhart, K. \& Karzai, W. (2001). Anti-tumor necrosis factor therapy in sepsis: update on clinical trials and lessons learned. Crit Care Med, 29(S), 121-125.

Reyes, W., Brimioulle, S. \& Vincent, J. (1999). Septic shock without documented infection: an uncommon entity with a high mortality. Int Care Med, 25, 1267-1270.

Riedemann, N., Neff, T., Guo, R., Bernacki, K., Laudes, I., Sarma, J., Lambris, J. \& Ward, P. (2003). Protective effects of IL-6 blockade in sepsis are linked to reduced C5a receptor expression. J. Immunol. 170, 503-507. 
Roberts, A. B. \& Sporn, M. (1996). Transforming growth factor- $\beta$. In Clark, R. A. F., ed. The Molecular and Cellular Biology of Wound Repair. New York, Plenum Press.

Ruiter, D. J., van der Meulen, J., Brouwer, A., Hummel, M., Mauw, B., van der Ploeg, J. \& Wisse, E. (1981). Uptake by liver cells of endotoxin following its intravenous injection. Lab Invest, 45, 38-45.

Santak, B., Radermacher, P., Iber, T., Adler, J., Wachter, U., Vassilev, D., Georgieff, M. \& Vogt, J. (1997). In vivo quantification of endotoxin-induced nitric oxide production in pigs from na15no3-infusion. Br.J Pharmacol. 122, 1605-1610.

Schneerson, R., Kubler-Kielb, J., Liu, T., Dai, Z., Leppla, S., Yergey, A., Backlund, P., Shiloach, J., Majadly, F. \& Robbins, J. (2003). Poly( $\gamma$-D-glutamic acid) protein conjugates induce IgG antibodies in mice to the capsule of Bacillus anthracis: A potential addition to the anthrax vaccine. Proc Natl Acad Sci. 100(15), 8945-8950.

Schultz, M., Sanchez, R., Hernandez, N. \& Hernandez, J. (2001). Nosocomial infection among immunosuppressed patients in the intensive care unit. Crit Care Nurs $Q, \mathbf{2 4 ( 2 )}$, $55-63$.

Shahfazand, S., Doyle, R., Ruoss, S., Weinacker, A. \& Raffin, T. (1999). Inhalational anthrax - epidemiology, diagnosis and management. Chest, 116, 1369-1376.

Solari, H. \& Natiello, M. (2003). Stochastic population dynamics : the poisson approximation. Phys Rev E, 67: 031918, 1-12.

Tilney, N., Bailey, G. \& Morgan, A. (1973). Sequential system failure after rupture of abdominal aortic aneurysms: an unsolved problem in postoperative care. Annals of Surgery, 178, 117-122.

Tjardes, T. \& Neugebauer, E. (2002). Sepsis research in the next millenium: concentrate on the software rather than the hardware. Shock, 17(1), 1-8.

van Kampen, N. (2003). Stochastic processes in physics and chemistry. North Holland Personal Library.

Villiger, P. M., Kusari, A., Ten Dijke, P. \& Lotz, M. (1993). IL-1 beta and IL-6 selectively induce transforming growth factor-beta isoforms in human articular chondrocytes. $J$ Immunol, 151, 3337-3344. 
Vincent, J. e. a. (2002). Reducing mortality in sepsis : New directions. Critical Care Forum, $1,1-10$.

Vodovotz, Y., Chesler, L., Chong, H., Kim, S., Simpson, J., DeGraff, W., Cox, G., Roberts, A., Wink, D. \& Barcellos-Hoff, M. (1999). Regulation of transforming growth factor- $\beta 1$ by nitric oxide. Cancer Res. 59, 2142-2149.

Vodovotz, Y., Chow, C., Kumar, R. \& Clermont, G. (2002). Simulating the roles of NO in septic shock. Nitric Oxide, 6, 484-484.

Volk, H. D., Reinke, P. \& Docke, W. (2000). Clinical aspects: from systemic inflammation to 'immunoparalysis'. Chem.Immunol. 74, 162-177.

Wang, H., Yang, H., Czura, C., Sama, A. \& Tracey, K. (2001). HMGB1 as a late mediator of lethal systemic inflammation. Am J Respir Crit Care Med. 164, 1768-1773.

Williams, R. \& Martinez, N. (2004). Stabilization of chaotic and non-permanent food-web dynamics. The European Phy Journal B, 38, 297-303.

Yodzis, P. \& Innes, S. (1992). Body size and consumer-resource dynamics. Amer Naturalist, 139, 1151-1175. 